Review Article

\title{
Recent Progress on the Description of Relativistic Spin: Vector Model of Spinning Particle and Rotating Body with Gravimagnetic Moment in General Relativity
}

\author{
Alexei A. Deriglazov ${ }^{1,2}$ and Walberto Guzmán Ramírez ${ }^{1}$ \\ ${ }^{1}$ Departamento de Matemática, ICE, Universidade Federal de Juiz de Fora, Juiz de Fora, MG, Brazil \\ ${ }^{2}$ Laboratory of Mathematical Physics, Tomsk Polytechnic University, Lenin Ave. 30, Tomsk 634050, Russia \\ Correspondence should be addressed to Alexei A. Deriglazov; alexei.deriglazov@uff.edu.br
}

Received 22 June 2017; Accepted 20 August 2017; Published 18 October 2017

Academic Editor: Eugen Radu

Copyright (c) 2017 Alexei A. Deriglazov and Walberto Guzmán Ramírez. This is an open access article distributed under the Creative Commons Attribution License, which permits unrestricted use, distribution, and reproduction in any medium, provided the original work is properly cited.

\begin{abstract}
We review the recent results on development of vector models of spin and apply them to study the influence of spin-field interaction on the trajectory and precession of a spinning particle in external gravitational and electromagnetic fields. The formalism is developed starting from the Lagrangian variational problem, which implies both equations of motion and constraints which should be presented in a model of spinning particle. We present a detailed analysis of the resulting theory and show that it has reasonable properties on both classical and quantum level. We describe a number of applications and show how the vector model clarifies some issues presented in theoretical description of a relativistic spin: (A) one-particle relativistic quantum mechanics with positive energies and its relation with the Dirac equation and with relativistic Zitterbewegung; (B) spin-induced noncommutativity and the problem of covariant formalism; (C) three-dimensional acceleration consistent with coordinate-independence of the speed of light in general relativity and rainbow geometry seen by spinning particle; (D) paradoxical behavior of the Mathisson-PapapetrouTulczyjew-Dixon equations of a rotating body in ultrarelativistic limit, and equations with improved behavior.
\end{abstract}

\section{Introduction}

Basic notions of Special and General Relativity have been formulated before the discovery of spin, so they describe the properties of space and time as they are seen by spinless testparticle. It is natural to ask whether these notions remain the same if the spinless particle is replaced by more realistic spinning test-particle. To analyze this issue, it is desirable to have a systematic formalism for semiclassical description of spinning degrees of freedom in relativistic (Poincaré invariant) and generally covariant theories.

Search for the relativistic equations that describe evolution of rotational degrees of freedom and their influence on the trajectory of a rotating body represents a problem with almost centenary history [1-8]. The equations are necessary for current applications of general relativity on various spacetime scales: for analysis of Lense-Thirring precession [9], for accounting spin effects in compact binaries and rotating black holes $[10,11]$, and in discussion of cosmological problems; see [12] and references therein. Closely related problem consists in establishing classical equations that could mimic quantum mechanics of an elementary particle with spin in a semiclassical approximation [13-17]. While the description of spin effects of relativistic electron is achieved in QED on the base of Dirac equation, the relationship among classical and quantum descriptions has an important bearing, providing interpretation of results of quantum field theory computations in usual terms: particles and their interactions. Semiclassical understanding of spin precession of a particle with an arbitrary magnetic moment is important in the development of experimental technics for measurements of anomalous magnetic moment $[18,19]$. In accelerator physics [20] it is important to control resonances leading to depolarization of a beam. In the case of vertex electrons carrying arbitrary angular momentum, semiclassical description can also be useful [21]. Basic equations of spintronics are based on 
heuristic and essentially semiclassical considerations [22, 23]. It would be very interesting to obtain them from first principles, that is, from equations of motion of a spinning particle.

Hence the further development of classical models of relativistic spinning particles/bodies represents an actual task. A review of the achievements in this fascinating area before 1968 can be found in the works of Dixon [6] and in the book of Corben [16]. Contrary to these works, where the problem was discussed on the level of equations of motion, our emphasis has been placed on the Lagrangian and Hamiltonian variational formulations for the description of rotational degrees of freedom. Taking a variational problem as the starting point, we avoid the ambiguities and confusion, otherwise arising in the passage from Lagrangian to Hamiltonian description and vice versa. Besides, it essentially fixes the possible form of interaction with external fields. In this review we show that so called vector model of spin represents a unified conceptual framework, allowing collecting and tying together a lot of remarkable ideas, observations, and results accumulated on the subject after 1968.

The present review article is based mainly on the recent works [24-33]. In [24] we constructed final Lagrangian for a spinning particle with an arbitrary magnetic moment. In [25] we presented the Lagrangian minimally interacting with gravitational field, while in $[26,27]$ it has been extended to the case of nonminimal interaction through the gravimagnetic moment. In all cases, our variational problem leads to both dynamical equations of motion and appropriate constraints; the latter guarantee the fixed value of spin, as well as the spin supplementary condition $S^{\mu \nu} p_{v}=0$. The works [27-33] are devoted to some applications of the vector model to various classical and quantum-mechanical problems.

We have not tried to establish a variational problem of the most general form $[34,35]$. Instead, the emphasis has been placed on the variational problem leading to the equations which are widely considered the most promising candidates for description of spinning particles in external fields. For the case of electromagnetic field, the vector model leads to a generalization of approximate equations of Frenkel and Bargmann and Michel and Telegdi (BMT) to the case of an arbitrary field. Here the strong restriction on possible form of equations is that the reasonable model should be in correspondence with the Dirac equation. In this regard, the vector model is of interest because it yields relativistic quantum mechanics with positive-energy states and is closely related to the Dirac equation.

Concerning the equations of a rotating body in general relativity, the widely assumed candidates are the MathissonPapapetrou-Tulczyjew-Dixon (MPTD) equations. While our vector model has been constructed as a semiclassical model of an elementary spin one-half particle, it turns out to be possible to apply it to the case: the vector model with minimal spin-gravity interaction and properly chosen parameters (mass and spin, see below) yields Hamiltonian equations equivalent to the MPTD equations. In the Lagrangian counterpart of MPTD equations emerges the term, which can be thought as an effective metric generated along the worldline by the minimal coupling. This leads to certain problems if we assume that MPTD equations remain applicable in the ultrarelativistic limit. In particular, three-dimensional acceleration of MPTD particle increases with velocity and becomes infinite in the limit. Therefore we examine the nonminimal interaction; this gives a generalization of MPTD equations to the case of a rotating body with gravimagnetic moment [36]. We show that a rotating body with unit gravimagnetic moment has an improved behavior in the ultrarelativistic regime and is free from the problems detected in MPTD equations.

Notation 1. Our variables are taken in arbitrary parametrization $\tau$; then $\dot{x}^{\mu}=d x^{\mu} / d \tau$. The square brackets mean antisymmetrization, $\omega^{[\mu} \pi^{\nu]}=\omega^{\mu} \pi^{\nu}-\omega^{\nu} \pi^{\mu}$. For the fourdimensional quantities we suppress the contracted indexes and use the notation $\dot{x}^{\mu} G_{\mu \nu} \dot{x}^{\nu}=\dot{x} G \dot{x}, N^{\mu}{ }_{\nu} \dot{x}^{\nu}=(N \dot{x})^{\mu}$, $\omega^{2}=g_{\mu \nu} \omega^{\mu} \omega^{\nu}$, and $\mu, \nu=0,1,2,3$. Notations for the scalar functions constructed from second-rank tensors are $\theta S=$ $\theta^{\mu \nu} S_{\mu \nu}, S^{2}=S^{\mu \nu} S_{\mu \nu}$. When we work in four-dimensional Minkowski space with coordinates $x^{\mu}=\left(x^{0}=c t, x^{i}\right)$, we use the metric $\eta_{\mu \nu}=(-,+,+,+)$, then $\dot{x} \omega=\dot{x}^{\mu} \omega_{\mu}=-\dot{x}^{0} \omega^{0}+\dot{x}^{i} \omega^{i}$, and so on. Levi-Civita tensors in four and three dimensions are defined by $\epsilon^{0123}=-\epsilon_{0123}=1$ and $\epsilon^{i j k}=\epsilon_{i j k}=1$. Suppressing the indexes of three-dimensional quantities, we use bold letters, $v^{i} \gamma_{i j} a^{j}=\mathbf{v} \gamma \mathbf{a}, v^{i} G_{i \mu} v^{\mu}=\mathbf{v} G v, i, j=1,2,3$, and so on.

The covariant derivative is $\nabla P^{\mu}=d P^{\mu} / d \tau+\Gamma_{\alpha \beta}^{\mu} \dot{x}^{\alpha} P^{\beta}$. The tensor of Riemann curvature is $R_{\lambda \mu \nu}^{\sigma}=\partial_{\mu} \Gamma_{\lambda \nu}^{\sigma}-\partial_{\nu} \Gamma_{\lambda \mu}^{\sigma}+$ $\Gamma_{\beta \mu}^{\sigma} \Gamma_{\lambda \nu}^{\beta}-\Gamma_{\beta \nu}^{\sigma} \Gamma_{\lambda \mu}^{\beta}$.

Electromagnetic field:

$$
\begin{aligned}
F_{\mu \nu} & =\partial_{\mu} A_{\nu}-\partial_{\nu} A_{\mu}=\left(F_{0 i}=-E_{i}, F_{i j}=\epsilon_{i j k} B_{k}\right), \\
E_{i} & =-\frac{1}{c} \partial_{t} A_{i}+\partial_{i} A_{0} \\
B_{i} & =\frac{1}{2} \epsilon_{i j k} F_{j k}=\epsilon_{i j k} \partial_{j} A_{k} .
\end{aligned}
$$

\section{Lagrangian Form of Mathisson- Papapetrou-Tulczyjew-Dixon Equations of a Rotating Body}

Equations of motion of a rotating body in curved background are formulated usually in the multipole approach to description of the body; see [37] for the review. In this approach, the energy-momentum of the body is modelled by a set of quantities called multipoles. Then the conservation law for the energy-momentum tensor, $\nabla_{\mu} T^{\mu \nu}=0$, implies certain equations for the multipoles. The first results were reported by Mathisson [1,2] and Papapetrou [4]. They have taken the approximation which involves only first two terms (the poledipole approximation). Manifestly covariant equations were formulated by Tulczyjew [5] and Dixon [6]. In the current literature they usually appear in the form given by Dixon ((6.31)-(6.33) in [6]), we will refer to them as MathissonPapapetrou-Tulczyjew-Dixon equations. 
We discuss MPTD equations in the form studied by Dixon (our $S$ is twice that of Dixon)

$$
\begin{aligned}
\nabla P^{\mu} & =-\frac{1}{4} R^{\mu}{ }_{\nu \alpha \beta} S^{\alpha \beta} \dot{x}^{\nu} \equiv-\frac{1}{4}(\theta \dot{x})^{\mu}, \\
\nabla S^{\mu \nu} & =2\left(P^{\mu} \dot{x}^{\nu}-P^{\nu} \dot{x}^{\mu}\right), \\
S^{\mu \nu} P_{\nu} & =0 .
\end{aligned}
$$

In the multipole approach, $x^{\mu}(\tau)$ is called representative point of the body; we take it in arbitrary parametrization $\tau$ (contrary to Dixon, we do not assume the proper-time parametrization (we will be interested in ultrarelativistic behavior of a body. The proper-time parametrization has no sense when $v \rightarrow c$ ); that is, we do not add the equation $g_{\mu \nu} \dot{x}^{\mu} \dot{x}^{\nu}=-c^{2}$ to the system above). $S^{\mu \nu}(\tau)$ is associated with inner angular momentum, and $P^{\mu}(\tau)$ is called momentum. The first-order equations (2) and (3) appear in the pole-dipole approximation, while the algebraic equation (4) has been added by hand. In the multipole approach it is called the spin supplementary condition (SSC) and corresponds to the choice of representative point as the center of mass $[5,6,8]$. After adding (4) to the system, the number of equations coincides with the number of variables.

Since we are interested in the influence of spin on the trajectory of a particle, we eliminate the momenta from MPTD equations, thus obtaining a second-order equation for the representative point $x^{\mu}(\tau)$. The most interesting property of the resulting equation is the emergence of an effective metric $G_{\mu \nu}$ instead of the original metric $g_{\mu \nu}$.

Let us start from some useful consequences of the system (2)-(4). Take derivative of the constraint, $\nabla\left(S^{\mu \nu} P_{\nu}\right)=0$, and use (2) and (3); this gives the expression

$$
(P \dot{x}) P^{\mu}=P^{2} \dot{x}^{\mu}+\frac{1}{8}(S \theta \dot{x})^{\mu},
$$

which can be written in the form

$$
P^{\mu}=\frac{P^{2}}{(P \dot{x})}\left(\delta^{\mu}{ }_{\nu}+\frac{1}{8 P^{2}}(S \theta)^{\mu}{ }_{\nu}\right) \dot{x}^{\nu} \equiv \frac{P^{2}}{(P \dot{x})} \widetilde{T}^{\mu}{ }_{\nu} \dot{x}^{\nu} .
$$

Contract (5) with $g_{\mu \alpha} \dot{x}^{\alpha}$. Taking into account the fact that $(P \dot{x})<0$, this gives $(P \dot{x})=-\sqrt{-P^{2}} \sqrt{-\dot{x} \widetilde{T} \dot{x}}$. Using this in (6) we obtain

$$
P^{\mu}=\frac{\sqrt{-P^{2}}}{\sqrt{-\dot{x} \widetilde{T} \dot{x}}} \widetilde{T}^{\mu}{ }_{\nu} \dot{x}^{\nu}, \quad \widetilde{T}^{\mu}{ }_{\nu}=\delta^{\mu}{ }_{\nu}+\frac{1}{8 P^{2}}(S \theta)^{\mu}{ }_{\nu} .
$$

Contracting (3) with $S_{\mu \nu}$ and using (4) we obtain $(d / d \tau)\left(S^{\mu \nu} S_{\mu \nu}\right)=0$; that is, square of spin is a constant of motion. Contraction of (5) with $P_{\mu}$ gives $(P S \theta \dot{x})=0$. Contraction of (5) with $(\dot{x} \theta)_{\mu}$ gives $(P \theta \dot{x})=0$. Contraction of (2) with $P_{\mu}$, gives $(d / d \tau)\left(P^{2}\right)=-(1 / 2)(P \theta \dot{x})=0$; that is, $P^{2}$ is one more constant of motion, say $k, \sqrt{-P^{2}}=k=$ const (in our vector model developed below this is fixed as $k=m c$ ). Substituting (7) into the equations (2)-(4) we now can exclude $P^{\mu}$ from these equations, modulo to the constant of motion $k=\sqrt{-P^{2}}$.
Thus, square of momentum can not be excluded from the system (2)-(5), that is MPTD equations in this form do not represent a Hamiltonian system for the pair $x^{\mu}, P^{\mu}$. To improve this point, we note that (7) acquires a conventional form (as the expression for conjugate momenta of $x^{\mu}$ in the Hamiltonian formalism), if we add to the system (2)-(4) one more equation, which fixes the remaining quantity $P^{2}$. To see how the equation could look, we note that for nonrotating body (pole approximation) we expect equations of motion of spinless particle, $\nabla p^{\mu}=0, p^{\mu}=(\widetilde{m} c / \sqrt{-\dot{x} g \dot{x}}) \dot{x}^{\mu}$, and $p^{2}+$ $(\widetilde{m} c)^{2}=0$. Independent equations of the system (2)-(5) in this limit read $\nabla P^{\mu}=0, P^{\mu}=\left(\sqrt{-P^{2}} / \sqrt{-\dot{x} g \dot{x}}\right) \dot{x}^{\mu}$. Comparing the two systems, we see that the missing equation is the massshell condition $P^{2}+(\widetilde{m} c)^{2}=0$. Returning to the pole-dipole approximation, an admissible equation should be $P^{2}+(\widetilde{m} c)^{2}+$ $f(S, \ldots)=0$, where $f$ must be a constant of motion. Since the only constant of motion in arbitrary background is $S^{2}$, we write (we could equally start with $P^{2}+(\widetilde{m} c)^{2}+f\left(S^{2}, P^{2}\right)=0$; assuming that this equation can be resolved with respect to $P^{2}$, we arrive essentially at the same expression)

$$
\begin{aligned}
& P^{2}=-(\widetilde{m} c)^{2}-f\left(S^{2}\right) \equiv-(m c)^{2}, \\
& \text { where } m \equiv \sqrt{\widetilde{m}^{2}+\frac{f\left(s^{2}\right)}{c^{2}}} .
\end{aligned}
$$

With this value of $P^{2}$, we can exclude $P^{\mu}$ from MPTD equations, obtaining closed system with second-order equation for $x^{\mu}$ (so we refer to the resulting equations as Lagrangian form of MPTD equations). We substitute (7) into (2)-(4); this gives the system

$$
\begin{aligned}
\nabla \frac{(\widetilde{T} \dot{x})^{\mu}}{\sqrt{-\dot{x} \widetilde{T} \dot{x}}} & =-\frac{1}{4 \sqrt{-P^{2}}}(\theta \dot{x})^{\mu}, \\
\nabla S^{\mu \nu} & =\frac{1}{4 \sqrt{-P^{2}} \sqrt{-\dot{x} \widetilde{T} \dot{x}}} \dot{x}^{[\mu}(S \theta \dot{x})^{\nu]}, \\
(S S \theta \dot{x})^{\mu} & =-8 P^{2}(S \dot{x})^{\mu},
\end{aligned}
$$

where (8) is implied. They determine evolution of $x^{\mu}$ and $S^{\mu \nu}$ for each given function $f\left(S^{2}\right)$.

It is convenient to introduce the symmetric matrix $G$ composed of the "tetrad field" $\widetilde{T}$ of (7):

$$
G_{\mu \nu}=g_{\alpha \beta} \widetilde{T}_{\mu}^{\alpha} \widetilde{T}_{\nu}^{\beta}=g_{\mu \nu}+h_{\mu \nu}(S) .
$$

Since this is composed of the original metric $g_{\mu \nu}$ plus (spin and field-dependent) contribution $h_{\mu \nu}$, we call $G$ the effective metric produced along the world-line by interaction of spin with gravity. Eq. (11) implies the identity

$$
\dot{x} \widetilde{T} \dot{x}=\dot{x} G \dot{x},
$$

so we can replace $\sqrt{-\dot{x} \widetilde{T} \dot{x}}$ in (9)-(11) by $\sqrt{-\dot{x} G \dot{x}}$. In particular, (9) reads

$$
\nabla \frac{(\widetilde{T} \dot{x})^{\mu}}{\sqrt{-\dot{x} G \dot{x}}}=-\frac{1}{4 \sqrt{-P^{2}}}(\theta \dot{x})^{\mu}
$$


Adding the consequences found above to the MPTD equations (2)-(4), we have the system

$$
\begin{gathered}
P^{\mu}=\frac{\sqrt{-P^{2}}}{\sqrt{-\dot{x} G \dot{x}}}(\widetilde{T} \dot{x})^{\mu}, \\
\nabla P^{\mu}=-\frac{1}{4}(\theta \dot{x})^{\mu}, \\
\nabla S^{\mu \nu}=2 P^{[\mu} \dot{x}^{\nu]}, \\
S^{\mu \nu} P_{\nu}=0, \\
P^{2}+(m c)^{2}+f\left(S^{2}\right)=0, \\
S^{2}, m\left(S^{2}\right) \text { are constants of motion, }
\end{gathered}
$$

with $\widetilde{T}$ given in (7). In Section 13.2 we will see that they essentially coincide with Hamiltonian equations of our spinning particle with vanishing gravimagnetic moment.

Let us finish this section with the following comment. Our discussion in the next two sections will be around the factor $\dot{x} G \dot{x}$, where the effective metric $G_{\mu \nu}$ appeared. The equation for trajectory (14) became singular for the particle's velocity which annihilates this factor, $\dot{x} G \dot{x}=0$. We call this the critical velocity. The observer-independent scale $c$ of special relativity is called, as usual, the speed of light. The singularity determines behavior of the particle in ultrarelativistic limit. To clarify this point, consider the standard equations of a spinless particle interacting with electromagnetic field in the physical time parametrization $x^{\mu}(t)=(c t, \mathbf{x}(t)),\left(\dot{x}^{\mu} / \sqrt{c^{2}-\mathbf{v}^{2}}\right) \cdot=\left(e / m c^{2}\right) F^{\mu}{ }_{\gamma} \dot{x}^{\nu}$. Then the factor is just $c^{2}-\mathbf{v}^{2}$; that is, critical speed coincides with the speed of light. Rewriting the equations of motion in the form of second law of Newton, we find an acceleration. For the case, the longitudinal acceleration reads $a_{\|}=\mathbf{v a}=\left(e\left(c^{2}-\right.\right.$ $\left.\left.\mathbf{v}^{2}\right)^{3 / 2} / m c^{3}\right)(\mathbf{E v})$; that is, the factor, elevated in some degree, appears on the right hand side of the equation and thus determines the value of velocity at which the longitudinal acceleration vanishes, $a_{\|} \stackrel{v \rightarrow c}{\longrightarrow} 0$. So the singularity implies (we point out that the factor can be hidden using the singular parametrization; for instance, in the proper-time parametrization this would be encoded into the definition of $d s, d s=\sqrt{c^{2}-\mathbf{v}^{2}} d t$ ) that during its evolution in the external field the spinless particle can not exceed the speed of light $c$.

\section{Three-Dimensional Acceleration and Speed of Light in General Relativity}

The ultrarelativistic behavior of MPTD particle in an arbitrary gravitational field will be analyzed by estimation of three-acceleration as $v \rightarrow c$. Let us discuss the necessary notions.

By construction of Lorentz transformations, the speed of light in special relativity is an observer-independent quantity. In the presence of gravity, we replace the Minkowski space by a four-dimensional pseudo-Riemann manifold

$$
\mathbf{M}^{(1,3)}=\left\{x^{\mu}, g_{\mu \nu}\left(x^{\rho}\right), g_{00}<0\right\} .
$$

To discuss the physics behind this abstract four-dimensional construction, we should establish a correspondence between the quantities computed in an arbitrary coordinates of the Riemann space and the three-dimensional quantities used by an observer in his laboratory. In particular, in a curved space we replace the Lorentz transformations on the generalcoordinate ones, so we need to ensure the coordinateindependence of the speed of light for that case. It turns out that this essentially determines the relationship between the four-dimensional and three-dimensional geometries [38]. We first recall the most simple part of this problem, which consist in determining basic differential quantities of threedimensional geometry: infinitesimal distances, time intervals, and velocity [38]. Then we define the three-dimensional acceleration which guarantees that a particle, propagating along a four-dimensional geodesic, can not exceed the speed of light. This gives us the necessary tool for discussion of a fast moving body.

The behavior of ultrarelativistic particles turns out to be important for analysis of near horizon geometry of extremal black holes (see [39]) and for accurate accounting of the corresponding corrections to geodesic motion near black hole [40-48].

3.1. Coordinate-Independence of Speed of Light. Consider an observer that labels events by some coordinates of pseudoRiemann space (18) to describe the motion of a point particle in a gravitational field with given metric $g_{\mu \nu}$. Formal definitions of the three-dimensional quantities can be obtained representing four-interval in $1+3$ block-diagonal form

$$
\begin{aligned}
-d s^{2}= & g_{\mu \nu} d x^{\mu} d x^{\nu} \\
= & -c^{2}\left[\frac{\sqrt{-g_{00}}}{c}\left(d x^{0}+\frac{g_{0 i}}{g_{00}} d x^{i}\right)\right]^{2} \\
& +\left(g_{i j}-\frac{g_{0 i} g_{0 j}}{g_{00}}\right) d x^{i} d x^{j} .
\end{aligned}
$$

This prompts introducing infinitesimal time interval and distance as follows:

$$
\begin{gathered}
d t=\frac{\sqrt{-g_{00}}}{c}\left(d x^{0}+\frac{g_{0 i}}{g_{00}} d x^{i}\right) \equiv-\frac{g_{0 \mu} d x^{\mu}}{c \sqrt{-g_{00}}} . \\
d l^{2}=\left(g_{i j}-\frac{g_{0 i} g_{0 j}}{g_{00}}\right) d x^{i} d x^{j} \equiv \gamma_{i j} d x^{i} d x^{j} .
\end{gathered}
$$

Therefore the conversion factor between intervals of the coordinate time $d x^{0} / c$ and the time $d t$ measured by laboratory clock is

$$
\frac{d t}{d x^{0}}=\frac{\sqrt{-g_{00}}}{c}\left(1+\frac{g_{0 i}}{g_{00}} \frac{d x^{i}}{d x^{0}}\right) .
$$

From (20) it follows that laboratory time coincides with coordinate time in the synchronous coordinate systems where metric acquires the forms $g_{00}=1$ and $g_{0 i}=0$. If metric is not of this form, we can not describe trajectory $\mathbf{x}(t)$ using the laboratory time $t$ as a global parameter. But we can 
describe it by the function $\mathbf{x}\left(x^{0}\right)$ and then determine various differential characteristics (such as velocity and acceleration) using the conversion factor (22). For instance, three-velocity of the particle is

$$
v^{i}=\left(\frac{d t}{d x^{0}}\right)^{-1} \frac{d x^{i}}{d x^{0}}
$$

so it is convenient to introduce the notation

$$
\begin{aligned}
\partial_{t} & \equiv\left(\frac{d t}{d x^{0}}\right)^{-1} \frac{\partial}{\partial x^{0}}, \\
\frac{d}{d t} & \equiv\left(\frac{d t}{d x^{0}}\right)^{-1} \frac{d x^{i}}{d x^{0}}, \\
\text { then, symbolically, } v^{i} & =\frac{d x^{i}}{d t}, \\
v & =\frac{d l}{d t} .
\end{aligned}
$$

The definitions of $\mathbf{v}$ and $v$ are consistent: $v^{2}=(d l / d t)^{2}=$ $\mathbf{v}^{2}=v^{i} \gamma_{i j} v^{j}$. Three-dimensional geometry is determined by the metric $\gamma_{i j}\left(x^{0}, \mathbf{x}\right)$. In particular, square of length of a vector is given by $\mathbf{v} \gamma \mathbf{v}=v^{i} \gamma_{i j} v^{j}$. Using these notations, the infinitesimal interval acquires the form similar to special relativity

$$
d s^{2}=c^{2} d t^{2}-d l^{2}=d t^{2}\left(c^{2}-\mathbf{v} \gamma \mathbf{v}\right)
$$

This equality holds in any coordinate system $x^{\mu}$. Hence a particle with the propagation law $d s^{2}=0$ has the speed $\mathbf{v}^{2}=c^{2}$, and this is a coordinate-independent statement. The value of the constant $c$, introduced by hand, is fixed from the flat limit: (20) implies $d t=c d x^{0}$ when $g_{\mu \nu} \rightarrow \eta_{\mu \nu}$.

These rather formal tricks are based [38] on the notion of simultaneity in general relativity and on the analysis of flat limit. The four-interval of special relativity has direct physical interpretation in two cases. First, for two events which occur at the same point, the four-interval is proportional to time interval, $d t=-(d s / c)$. Second, for simultaneous events, the four-interval coincides with distance, $d l=d s$. Assuming that the same holds in general relativity, let us analyze infinitesimal time interval and distance between two events with coordinates $x^{\mu}$ and $x^{\mu}+d x^{\mu}$. The world-line $y^{\mu}=$ $\left(y^{0}, \mathbf{y}=\right.$ const $)$ is associated with laboratory clock placed at the spatial point $\mathbf{y}$. So the time interval between the events $\left(y^{0}, \mathbf{y}\right)$ and $\left(y^{0}+d y^{0}, \mathbf{y}\right)$ measured by the clock is

$$
d t=-\frac{d s}{c}=\frac{\sqrt{-g_{00}}}{c} d y^{0} .
$$

Consider the event $x^{\mu}$ infinitesimally closed to the world-line $\left(y^{0}, \mathbf{y}=\right.$ const $)$. To find the event on the world-line which is simultaneous with $x^{\mu}$, we first look for the events $y_{(1)}^{\mu}$ and $y_{(2)}^{\mu}$ which have null-interval with $x^{\mu}, d s\left(x^{\mu}, y_{(a)}^{\mu}\right)=0$. The equation $g_{\mu \nu} d x^{\mu} d x^{\nu}=0$ with $d x^{\mu}=x^{\mu}-y^{\mu}$ has two solutions

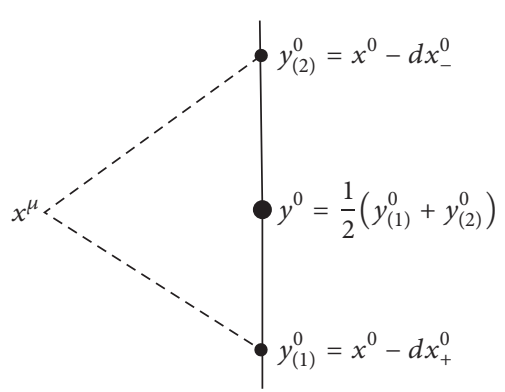

FIgure 1: Definition of simultaneous events. The vertical line represents a world-line of the laboratory clock. The points $y_{(1)}^{0}$ and $y_{(2)}^{0}$ have null-interval with $x^{\mu}$. Then the middle point $y^{0}$ represents the event simultaneous with $x^{\mu}$.

$d x_{ \pm}^{0}=g_{0 i} d x^{i} /-g_{00} \pm \sqrt{d \mathbf{x} \gamma d \mathbf{x}} / \sqrt{-g_{00}}$ and then $y_{(1)}^{0}=x^{0}-d x_{+}^{0}$ and $y_{(2)}^{0}=x^{0}-d x_{-}^{0}$. Second, we compute the middle point

$$
y^{0}=\frac{1}{2}\left(y_{(1)}^{0}+y_{(2)}^{0}\right)=x^{0}+\frac{g_{0 i} d x^{i}}{g_{00}} \text {. }
$$

By definition (in the flat limit the sequence $y_{(1)}^{\mu}, x^{\mu}, y_{(2)}^{\mu}$ of events can be associated with emission, reflection, and absorption of a photon with the propagation law $d s=0$; then the middle point (27) should be considered simultaneous with $\left.x^{0}\right)$, the event $\left(y^{0}, \mathbf{y}\right)$ with the null-coordinate $(27)$ is simultaneous with the event $\left(x^{0}, \mathbf{x}\right)$; see Figure 1 . By this way we synchronized clocks at the spatial points $\mathbf{x}$ and $\mathbf{y}$. According to (27), the simultaneous events have different null-coordinates, and the difference $d x^{0}$ obeys the equation

$$
d x^{0}+\frac{g_{0 i} d x^{i}}{g_{00}}=0 .
$$

Consider a particle which propagated from $x^{\mu}$ to $x^{\mu}+d x^{\mu}$. Let us compute time interval and distance between these two events. According to (27), the event

$$
\left(x^{0}+d x^{0}+\frac{g_{0 i} d x^{i}}{g_{00}}, \mathbf{x}\right)
$$

at the spatial point $\mathbf{x}$ is simultaneous with $x^{\mu}+d x^{\mu}$; see Figure 2. Equation (29) determines the event $A$ (at spatial point $\mathbf{x}$ ) simultaneous with $x^{\mu}+d x^{\mu}$. So the time interval between $x^{\mu}$ and $x^{\mu}+d x^{\mu}$ coincides with the interval between $x^{\mu}$ e $A$ and is given by (30). Distance between $x^{\mu}$ and $x^{\mu}+d x^{\mu}$ coincides with the distance between $x^{\mu}+d x^{\mu}$ and $A$; the latter is given in (31).

According to (26) and (27), the time interval between the events $x^{\mu}$ and (29) is

$$
d t=\frac{\sqrt{-g_{00}}}{c}\left(d x^{0}+\frac{g_{0 i}}{g_{00}} d x^{i}\right) .
$$

Since the events $x^{\mu}+d x^{\mu}$ and (29) are simultaneous, this equation gives also the time interval between $x^{\mu}$ and $x^{\mu}+$ $d x^{\mu}$. Further, the difference of coordinates between the events 


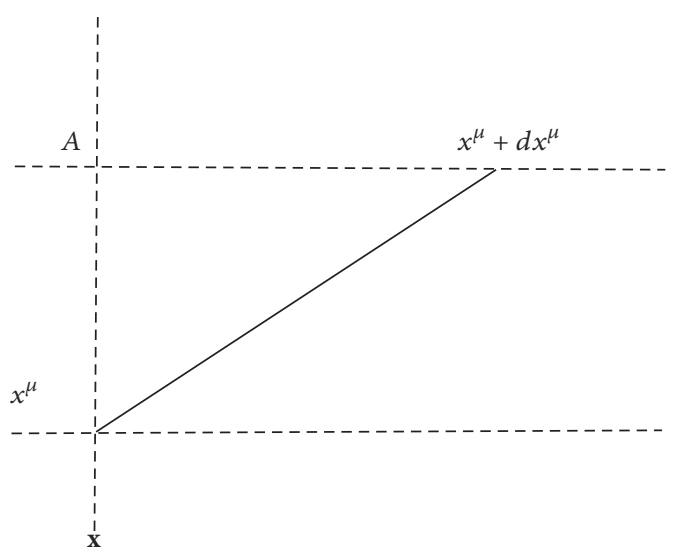

Figure 2: Time and distance between the events $x^{\mu}$ and $x^{\mu}+$ $d x^{\mu}$. Equation (29) determines the event $A$ (at spatial point $x$ ) simultaneous with $x^{\mu}+d x^{\mu}$. So the time interval between $x^{\mu}$ and $x^{\mu}+d x^{\mu}$ coincides with the interval between $x^{\mu}$ and $A$ and is given by (30). Distance between $x^{\mu}$ and $x^{\mu}+d x^{\mu}$ coincides with the distance between $x^{\mu}+d x^{\mu}$ and $A$; the latter is given in (31).

$x^{\mu}+d x^{\mu}$ and (29) is $d z^{\mu}=\left(-\left(g_{0 i} d x^{i} / g_{00}\right), d x^{i}\right)$. As they are simultaneous, the distance between them is

$$
\begin{aligned}
d l^{2} & =-d s^{2}=g_{\mu \nu} d z^{\mu} d z^{\nu}=\left(g_{i j}-\frac{g_{0 i} g_{0 j}}{g_{00}}\right) d x^{i} d x^{j} \\
& \equiv \gamma_{i j} d x^{i} d x^{j} .
\end{aligned}
$$

Since (29) occurs at the same spatial point as $x^{\mu}$, this equation gives also the distance between $x^{\mu}$ and $x^{\mu}+d x^{\mu}$. Equations (30) and (31) coincide with the formal definitions presented above, (20) and (21).

\subsection{Three-Dimensional Acceleration and Maximum Speed of a} Particle in Geodesic Motion. We now turn to the definition of three-acceleration. Point particle in general relativity follows a geodesic line, and we expect that during its evolution in gravitational field the particle can not reach the speed of light. This implies that longitudinal acceleration should vanish when speed of the particle approximates to $c$. To analyze this, we first use geodesic equation to obtain the derivative $d v^{i} / d t$ of coordinate of the velocity vector.

If we take the proper time to be the parameter, geodesics obey the system

$$
\begin{aligned}
\nabla_{s} \frac{d x^{\mu}}{d s} & \equiv \frac{d^{2} x^{\mu}}{d s^{2}}+\Gamma_{\alpha \beta}^{\mu} \frac{d x^{\alpha}}{d s} \frac{d x^{\beta}}{d s}=0, \\
g_{\mu \nu} \frac{d x^{\mu}}{d s} \frac{d x^{\nu}}{d s} & =-1
\end{aligned}
$$

where

$$
\Gamma_{\alpha \beta}^{\mu}=\frac{1}{2} g^{\mu \nu}\left(\partial_{\alpha} g_{\nu \beta}+\partial_{\beta} g_{\alpha \nu}-\partial_{\nu} g_{\alpha \beta}\right)
$$

Due to this definition, the system (32) obeys the identity $g_{\mu \nu}\left(d x^{\mu} / d s\right) \nabla_{s}\left(d x^{\nu} / d s\right)=0$. The system in this parametrization has no sense for the case we are interested in, $d s^{2} \rightarrow 0$. So we rewrite it in arbitrary parametrization $\tau$

$$
\begin{aligned}
\frac{d \tau}{d s} \frac{d}{d \tau}\left(\frac{d \tau}{d s} \frac{d x^{\mu}}{d \tau}\right)+\left(\frac{d \tau}{d s}\right)^{2} \Gamma_{\alpha \beta}^{\mu}(g) \frac{d x^{\alpha}}{d \tau} \frac{d x^{\beta}}{d \tau}=0, \\
\frac{d \tau}{d s}=\frac{1}{\sqrt{-\dot{x} g \dot{x}}} ;
\end{aligned}
$$

this yields the equation of geodesic line in reparametrizationinvariant form

$$
\begin{aligned}
& \frac{1}{\sqrt{-\dot{x} g \dot{x}}} \frac{d}{d \tau}\left(\frac{\dot{x}^{\mu}}{\sqrt{-\dot{x} g \dot{x}}}\right) \\
& =-\Gamma^{\mu}{ }_{\alpha \beta}(g) \frac{\dot{x}^{\alpha}}{\sqrt{-\dot{x} g \dot{x}}} \frac{\dot{x}^{\beta}}{\sqrt{-\dot{x} g \dot{x}}} .
\end{aligned}
$$

Using the reparametrization invariance, we set $\tau=x^{0}$; then (20) and (22) imply $\sqrt{-\dot{x} g \dot{x}}=\left(d t / d x^{0}\right) \sqrt{c^{2}-\mathbf{v} \gamma \mathbf{v}}$, and spatial part of (35) reads

$$
\left(\frac{d t}{d x^{0}}\right)^{-1} \frac{d}{d x^{0}} \frac{v^{i}}{\sqrt{c^{2}-\mathbf{v} \gamma \mathbf{v}}}=-\frac{1}{\sqrt{c^{2}-\mathbf{v} \gamma \mathbf{v}}} \Gamma_{\mu \nu}^{i} \nu^{\mu} v^{\nu},
$$

where we have denoted

$$
v^{\mu}=\left(\frac{d t}{d x^{0}}\right)^{-1} \frac{d x^{\mu}}{d x^{0}}=\left(\left(\frac{d t}{d x^{0}}\right)^{-1}, \mathbf{v}\right) .
$$

Direct computation of the derivative on 1.h.s. of (36) leads to the desired expression

$$
\begin{aligned}
\left(\frac{d t}{d x^{0}}\right)^{-1} \frac{d v^{i}}{d x^{0}}= & -\frac{v^{i}}{2 c^{2}}\left(\mathbf{v} \partial_{t} \gamma \mathbf{v}\right) \\
& -\frac{v^{i}}{c^{2}}(\mathbf{v} \gamma)_{p} \widetilde{\Gamma}^{p}{ }_{j k}(\gamma) v^{j} v^{k} \\
& -\widetilde{M}^{i}{ }_{j} \Gamma^{j}{ }_{\mu \nu}(g) v^{\mu} v^{\nu},
\end{aligned}
$$

where

$$
\begin{aligned}
\widetilde{M}_{j}^{i} & =\delta^{i}{ }_{j}-\frac{v^{i}(\mathbf{v} \gamma)_{j}}{c^{2}}, \\
\text { then } \widetilde{M}_{j}^{i} v^{j} & =\frac{c^{2}-\mathbf{v} \gamma \mathbf{v}}{c^{2}} v^{i}, \\
(\mathbf{v} \gamma)_{i} \widetilde{M}_{j}^{i} & =\frac{c^{2}-\mathbf{v} \gamma \mathbf{v}}{c^{2}}(\mathbf{v} \gamma)_{j},
\end{aligned}
$$

and three-dimensional Christoffel symbols $\widetilde{\Gamma}_{j k}^{i}(\gamma)$ are constructed with help of three-dimensional metric $\gamma_{i j}\left(x^{0}, x^{k}\right)$, where $x^{0}$ is considered as a parameter

$$
\widetilde{\Gamma}_{j k}^{i}(\gamma)=\frac{1}{2} \gamma^{i n}\left(\partial_{j} \gamma_{n k}+\partial_{k} \gamma_{n j}-\partial_{n} \gamma_{j k}\right) .
$$


We have $g^{i j} \gamma_{j k}=\delta^{i}{ }_{k}$, so the inverse metric of $\gamma_{i j}$ turns out to be $\gamma^{i j}=g^{i j}$. Note that $\widetilde{M}^{i}{ }_{j} v^{j} \stackrel{|v| \rightarrow c}{\longrightarrow} 0$; that is, in the limit the matrix $\widetilde{M}$ turns into the projector on the plane orthogonal to v.

If we project the derivative (38) on the direction of motion, we obtain the expression

$$
\begin{aligned}
\left(\mathbf{v} \gamma \frac{d \mathbf{v}}{d t}\right)= & -\frac{(\mathbf{v} \gamma \mathbf{v})}{2 c^{2}}\left(\mathbf{v} \partial_{t} \gamma \mathbf{v}\right) \\
& -\frac{(\mathbf{v} \gamma \mathbf{v})}{c^{2}}(\mathbf{v} \gamma)_{p} \widetilde{\Gamma}^{p}{ }_{j k}(\gamma) v^{j} v^{k} \\
& -\frac{\sqrt{c^{2}-\mathbf{v} \gamma \mathbf{v}}}{c^{2}}(\mathbf{v} \gamma)_{j} \Gamma_{\mu \nu}^{j}(g) v^{\mu} v^{\nu} .
\end{aligned}
$$

Due to the first and second terms on r. h. s., this expression does not vanish as $\mathbf{v} \gamma \mathbf{v} \rightarrow c^{2}$. Note that this remains true for stationary metric, $g_{\mu \nu}(\mathbf{x})$, or even for static metric, $g_{00}=1$, $g_{0 i}=0$ ! The reason is that the derivative $d v^{i} / d t$ in our three-dimensional geometry consists of three contributions: variation rate of the vector field $\mathbf{v}$ itself, variation of basis in the passage from $\mathbf{x}$ to $\mathbf{x}+d \mathbf{x}$, and variation of the metric $\gamma_{i j}$ during the time interval $d t$. Excluding the last two contributions, we obtain the variation rate of velocity itself, that is, an acceleration

$$
\begin{aligned}
a^{i} & =\left(\frac{d t}{d x^{0}}\right)^{-1} \frac{d v^{i}}{d x^{0}}+\widetilde{\Gamma}_{j k}^{i}(\gamma) v^{j} v^{k}+\frac{1}{2}\left(\mathbf{v} \partial_{t} \gamma \gamma^{-1}\right)^{i} \\
& \equiv \nabla_{t} v^{i}+\frac{1}{2}\left(\mathbf{v} \partial_{t} \gamma \gamma^{-1}\right)^{i} .
\end{aligned}
$$

For the special case of stationary field, $g_{\mu \nu}(\mathbf{x})$, our definition reduces to that of Landau and Lifshitz; see page 251 in [38]. Complementing $d v^{i} / d t$ in (38) up to the acceleration, we obtain three-dimensional acceleration of the particle moving along the geodesic line (35):

$$
a^{i}=\widetilde{M}_{j}^{i}\left[\frac{1}{2}\left(\gamma^{-1} \partial_{t} \gamma \mathbf{v}\right)^{j}-\Gamma_{\mu \nu}^{j}(g) v^{\mu} v^{\nu}+\widetilde{\Gamma}_{k l}^{j}(\gamma) v^{k} v^{l}\right] .
$$

This is the second Newton law for geodesic motion. Contracting this with $(\mathbf{v} \gamma)_{i}$, we obtain the longitudinal acceleration

$$
\begin{aligned}
\mathbf{v} \gamma \mathbf{a} & =\left(1-\frac{(\mathbf{v} \gamma \mathbf{v})}{c^{2}}\right)\left[\frac{1}{2}\left(\mathbf{v} \partial_{t} \gamma \mathbf{v}\right)\right. \\
& \left.+(\mathbf{v} \gamma)_{i}\left[-\Gamma_{\mu \nu}^{i}(g) v^{\mu} v^{\nu}+\widetilde{\Gamma}_{k l}^{i}(\gamma) v^{k} v^{l}\right]\right] .
\end{aligned}
$$

This implies $\mathbf{v} \gamma \mathbf{a} \rightarrow 0$ as $\mathbf{v} \gamma \mathbf{v} \rightarrow c^{2}$.

Let us confirm that $c$ is the only special point of the function (44). Using (33), (21)-(25), and (40) and the identities

$$
\begin{aligned}
& \gamma_{i j} g^{j k}=\delta_{i}{ }^{k}, \\
& \gamma_{i j} g^{j 0}=-\frac{g_{0 i}}{g_{00}},
\end{aligned}
$$

we can present the right hand side of (44) in terms of initial metric as follows:

$$
\begin{aligned}
\mathrm{v} \gamma \mathbf{a} & =\frac{c^{2}-\mathbf{v} \gamma \mathbf{v}}{2 c \sqrt{-g_{00}}}\left\{\frac { c } { \sqrt { - g _ { 0 0 } } } \left[\left(\frac{d t}{d x^{0}}\right)^{-1} \partial_{0} g_{00}\right.\right. \\
& \left.+v^{k} \partial_{k} g_{00}\right]-\partial_{0} g_{00}\left(\frac{d t}{d x^{0}}\right)^{-2}-2 \partial_{0} g_{0 k}\left(\frac{d t}{d x^{0}}\right)^{-1} \\
& \left.\cdot v^{k}-\partial_{0} g_{k l} v^{k} v^{l}\right\} \equiv \frac{c^{2}-\mathbf{v} \gamma \mathbf{v}}{2 c \sqrt{-g_{00}}}\left\{\frac{c}{\sqrt{-g_{00}}} v^{\mu} \partial_{\mu} g_{00}\right. \\
& \left.-\partial_{0} g_{\mu \nu} v^{\mu} v^{\nu}\right\} .
\end{aligned}
$$

The quantity $v^{\mu}$ has been defined in (37). Excluding $v^{0}$ according to this expression, we obtain

$$
\begin{aligned}
\mathbf{v} \gamma \mathbf{a} & =\frac{c^{2}-\mathbf{v} \gamma \mathbf{v}}{2 \sqrt{-g_{00}}}\left\{\frac{v^{k} \partial_{k} g_{00}}{\sqrt{-g_{00}}}-2 \partial_{0}\left(\frac{g_{0 i}}{\sqrt{-g_{00}}}\right) v^{i}\right. \\
& \left.-\frac{1}{c} \partial_{0} \gamma_{i j} v^{i} v^{j}\right\} .
\end{aligned}
$$

For the stationary metric, $g_{\mu \nu}\left(x^{k}\right),(47)$ acquires a specially simple form

$$
\mathbf{v} \gamma \mathbf{a}=-\left(c^{2}-\mathbf{v} \gamma \mathbf{v}\right) \frac{v^{k} \partial_{k} g_{00}}{2 g_{00}} .
$$

This shows that the longitudinal acceleration has only one special point, $\mathbf{v} \gamma \mathbf{a} \rightarrow 0$ as $\mathbf{v} \gamma \mathbf{v} \rightarrow c^{2}$. Hence the spinless particle in the stationary gravitational field can not overcome the speed of light. Then the same is true in general case (46), at least for the metric which is sufficiently slowly varied in time.

While we have discussed the geodesic equation, the computation which leads to the formula (44) can be repeated for a more general equation. Using the factor $\sqrt{-\dot{x} g \dot{x}}$ we construct the reparametrization-invariant derivative

$$
D=\frac{1}{\sqrt{-\dot{x} g \dot{x}}} \frac{d}{d \tau} .
$$

Consider the reparametrization-invariant equation of the form

$$
D D x^{\mu}(\tau)=\mathscr{F}^{\mu}\left(D x^{\nu}, \ldots\right)
$$

and suppose that the three-dimensional geometry is defined by $g_{\mu \nu}$ according to (20)-(23). Then (50) implies the threeacceleration

$$
\begin{aligned}
a^{i}= & \widetilde{M}_{j}^{i}\left[\left(c^{2}-\mathbf{v} \gamma \mathbf{v}\right) \mathscr{F}^{j}+\widetilde{\Gamma}_{k l}^{j}(\gamma) v^{k} v^{l}\right] \\
& +\frac{1}{2}\left(\frac{d t}{d x^{0}}\right)^{-1}\left[\left(\mathbf{v} \partial_{0} \gamma \gamma^{-1}\right)^{i}-\frac{v^{i}}{c^{2}}\left(\mathbf{v} \partial_{0} \gamma \mathbf{v}\right)\right],
\end{aligned}
$$


and the longitudinal acceleration

$$
\begin{aligned}
\mathbf{v} \gamma \mathbf{a} & =\frac{\left(c^{2}-\mathbf{v} \gamma \mathbf{v}\right)^{2}}{c^{2}}(\mathbf{v} \gamma \mathscr{F}) \\
& +\frac{c^{2}-\mathbf{v} \gamma \mathbf{v}}{c^{2}}\left[(\mathbf{v} \gamma)_{i} \widetilde{\Gamma}_{k l}^{i}(\gamma) v^{k} v^{l}\right. \\
& \left.+\frac{1}{2}\left(\frac{d t}{d x^{0}}\right)^{-1}\left(\mathbf{v} \partial_{0} \gamma \mathbf{v}\right)\right]
\end{aligned}
$$

The spatial part of the force is $\mathscr{F}^{i}=\mathscr{F}^{i}\left(v^{\nu} / \sqrt{c^{2}-\mathbf{v} \gamma \mathbf{v}}\right)$, where $v^{\mu}$ is given by (37), and the connection $\widetilde{\Gamma}_{k l}^{i}(\gamma)$ is constructed with help of the three-dimensional metric $\gamma_{i j}=\left(g_{i j}-\right.$ $\left.g_{0 i} g_{0 j} / g_{00}\right)$ according to (40). For the geodesic equation in this notation we have $\mathscr{F}^{i}=-\Gamma_{\mu \nu}^{i}\left(\nu^{\mu} v^{\nu} /\left(c^{2}-\mathbf{v} \gamma \mathbf{v}\right)\right)$. With this $\mathscr{F}^{i}$ (51) and (52) coincide with (43) and (44).

Eq. (52) shows that potentially dangerous forces are of degree four or more, $\mathscr{F}^{j} \sim(D x)^{4}$.

3.3. Parallel Transport in Three-Dimensional Geometry. Now we consider an arbitrary vector/tensor field in the space with three-dimensional geometry determined by a nonstatic metric $\gamma_{i j}\left(x^{0}, \mathbf{x}\right)$. Variation rate of the field along a curve $\mathbf{x}\left(x^{0}\right)$ should be defined in such a way that it coincides with (42) for the velocity. Let us show how this definition follows from natural geometric requirements [32]. $1+3$ splitting preserves covariance of the formalism under the following subgroup of general-coordinate transformations: $x^{0}=x^{\prime 0}, x^{i}=x^{i}\left(x^{\prime j}\right)$. Under these transformations $g_{00}$ is a scalar function and $g_{0 i}$ is a vector while $g_{i j}$ and $\gamma_{i j}$ are tensors; then the conversion factor (22) is a scalar function and the velocity (23) is a vector. So it is convenient to introduce the usual covariant derivative $\nabla_{k}$ of a vector field $\xi^{i}\left(x^{0}, \mathbf{x}\right)$ in the direction of $x^{k}$

$$
\nabla_{k} \xi^{i}=\frac{\partial \xi^{i}}{\partial x^{k}}+\widetilde{\Gamma}_{k j}^{i}(\gamma) \xi^{j}
$$

with the Christoffel symbols (40). By construction, the metric $\gamma$ is covariantly constant, $\nabla_{k} \gamma_{i j}=0$. For the field $\xi^{i}\left(x^{0}, \mathbf{x}\left(x^{0}\right)\right)$ along the curve $\mathbf{x}\left(x^{0}\right)$ we have the covariant derivative in the direction of $x^{0}$

$$
\nabla_{0} \xi^{i}=\frac{d \xi^{i}}{d x^{0}}+\widetilde{\Gamma}_{j k}^{i}(\gamma) \frac{d x^{j}}{d x^{0}} \xi^{k}=\frac{\partial \xi^{i}}{\partial x^{0}}+\frac{d x^{k}}{d x^{0}} \nabla_{k} \xi^{i} .
$$

To define the variation rate of $\boldsymbol{\xi}$, we need the notion of a constant field (or, equivalently, the parallel-transport equation). In Euclidean space the scalar product of two constant fields does not depend on the point where it was computed. In particular, taking the scalar product along a line $\mathbf{x}\left(x^{0}\right)$, we have $\left(d / d x^{0}\right)(\xi, \boldsymbol{\eta})=0$. For the constant fields in our case it is natural to demand the same (necessary) condition: $\left(d / d x^{0}\right)\left[\xi^{i}\left(x^{0}\right) \gamma_{i j}\left(x^{0}, x^{i}\left(x^{0}\right)\right) \eta^{i}\left(x^{0}\right)\right]=0$. Taking into account the fact that $\nabla_{k} \gamma_{i j}=0$, this condition can be written as follows:

$$
\left(\nabla_{0} \xi+\frac{1}{2} \xi \partial_{0} \gamma \gamma^{-1}, \boldsymbol{\eta}\right)+\left(\xi, \nabla_{0} \boldsymbol{\eta}+\frac{1}{2} \gamma^{-1} \partial_{0} \gamma \boldsymbol{\eta}\right)=0
$$

This will be satisfied, if a constant field is defined by the equation

$$
\nabla_{0} \xi^{i}+\frac{1}{2}\left(\xi \partial_{0} \gamma \gamma^{-1}\right)^{i}=0
$$

This is the parallel-transport equation in our three-dimensional geometry. Deviation from the constant field is the variation rate. Hence, when the l.h.s. does not vanish, it gives the variation rate, which we write with respect to physical time:

$$
\nabla_{t} \xi^{i} \equiv\left(\frac{d t}{d x^{0}}\right)^{-1}\left[\nabla_{0} \xi^{i}+\frac{1}{2}\left(\mathbf{v} \partial_{0} \gamma \gamma^{-1}\right)^{i}\right]
$$

This result is in correspondence with the definition (42) for acceleration.

\section{Behavior of Ultrarelativistic MPTD Particle and the Rainbow Geometry Induced by Spin}

As we saw above, point particle in a gravitational field propagates along a geodesic line with the speed less then speed of light. Let us study the influence of rotational degrees of freedom on the trajectory of a fast spinning particle.

Using (8) and (13), we present MPTD equations (9)-(11) in the following form:

$$
\begin{aligned}
& S^{\mu}{ }_{\nu} \dot{x}^{\nu}-\frac{1}{8(m c)^{2}}(S S \theta \dot{x})^{\mu}=0, \\
& \nabla\left[\frac{\widetilde{T}^{\mu}{ }_{\nu} \dot{x}^{\nu}}{\sqrt{-\dot{x} G \dot{x}}}\right]=-\frac{1}{4 m c} R^{\mu}{ }_{\nu \beta} S^{\alpha \beta} \dot{x}^{\nu}, \\
& \nabla S^{\mu \nu}=\frac{1}{4 m c \sqrt{-\dot{x} G \dot{x}}} \dot{x}^{[\mu} S^{\nu] \sigma} \theta_{\sigma \alpha} \dot{x}^{\alpha} .
\end{aligned}
$$

The equations for trajectory and for precession of spin become singular at the critical velocity which obeys the equation

$$
\dot{x} G \dot{x}=0 \text {. }
$$

The singularity determines behavior of the particle in ultrarelativistic limit. The effective metric is composed of the original one plus (spin and field-dependent) contribution, $G=g+$ $h(S)$. So we need to decide which of them the particle probes as the space-time metric. Let us consider separately the two possibilities.

Let us use $g$ to define the three-dimensional geometry (20)-(23). This leads to two problems. The first problem is that the critical speed turns out to be slightly more than the speed of light. To see this, we use the supplementary spin condition (58) to write (61) in the form

$$
\begin{aligned}
-\left(\frac{d t}{d x^{0}}\right)^{2} \dot{x} G \dot{x} & =\left(c^{2}-\mathbf{v} \gamma \mathbf{v}\right)+\frac{1}{\left(2 m^{2} c^{2}\right)^{2}}(v \theta S S \theta v) \\
& =0,
\end{aligned}
$$


with $v^{\mu}$ defined in (37). Using $S^{\mu \nu}=2 \omega^{[\mu} \pi^{\nu]}$, we rewrite the last term as follows:

$$
\left(c^{2}-\mathbf{v} \gamma \mathbf{v}\right)+\frac{1}{\left(m^{2} c^{2}\right)^{2}}\left(\pi^{2}(\nu \theta \omega)^{2}+\omega^{2}(\nu \theta \pi)^{2}\right)=0 .
$$

As $\pi$ and $\omega$ are space-like vectors, the last term is nonnegative; this implies $\left|\mathbf{v}_{\mathrm{cr}}\right| \geq c$. Let us confirm that generally this term is nonvanishing function of velocity; then $\left|\mathbf{v}_{\mathrm{cr}}\right|>c$. Assuming the contrary, that this term vanishes at some velocity, then

$$
\begin{aligned}
& v \theta \omega=\theta_{0 i} \omega^{i}+\theta_{i 0} v^{i} \omega^{0}=0, \\
& v \theta \pi=\theta_{0 i} \pi^{i}+\theta_{i 0} v^{i} \pi^{0}=0 .
\end{aligned}
$$

We analyze these equations in the following special case. Consider a space with covariantly constant curvature $\nabla_{\mu} R_{\mu \nu \alpha \beta}=$ 0 . Then $(d / d \tau)\left(\theta_{\mu \nu} S^{\mu \nu}\right)=2 \theta_{\mu \nu} \nabla S^{\mu \nu}$, and using (60) we conclude that $\theta_{\mu \nu} S^{\mu \nu}$ is an integral of motion. We further assume that the only nonvanishing part is the electric part of the curvature, $R_{0 i 0 j}=K_{i j}$. Then the integral of motion acquires the form

$$
\theta_{\mu \nu} S^{\mu \nu}=2 K_{i j} S^{0 i} S^{0 j} .
$$

Let us take the initial conditions for spin such that $K_{i j} S^{0 i} S^{0 j} \neq$ 0 ; then this holds at any future instant. Contrary to this, the system (64) implies $K_{i j} S^{0 i} S^{0 j}=0$. Thus, the critical speed does not always coincide with the speed of light and, in general case, we expect that $\mathbf{v}_{\mathrm{cr}}$ is both field and spindependent quantity.

The second problem is that acceleration of MPTD particle grows up in the ultrarelativistic limit. In the spinless limit (59) turns into the geodesic equation. Spin causes deviations from the geodesic equation due to right hand side of this equation, as well as due to the presence of the tetrad field $\widetilde{T}$ and of the effective metric $G$ in the left hand side. Due to the dependence of the tetrad field on the spin-tensor $S$, the singularity presented in (60) causes the appearance of the term proportional to $1 / \sqrt{\dot{x} G \dot{x}}$ in the expression for longitudinal acceleration. In the result, the acceleration grows up to infinity as the particle's speed approximates to the critical speed. To see this, we separate derivative of $\widetilde{T}$ in (59):

$$
\begin{aligned}
\nabla\left[\frac{\dot{x}^{\mu}}{\sqrt{-\dot{x} G \dot{x}}}\right]= & -T_{\alpha}^{\mu}\left(\nabla \widetilde{T}_{\beta}^{\alpha}\right) \frac{\dot{x}^{\beta}}{\sqrt{-\dot{x} G \dot{x}}} \\
& -\frac{1}{4 m c} T^{\mu}{ }_{\nu}(\theta \dot{x})^{\nu},
\end{aligned}
$$

where $T$ is the inverse for $\widetilde{T}$. Using (60) we obtain

$$
\begin{aligned}
& {\left[\nabla \widetilde{T}_{\nu}^{\mu}{ }_{\nu} \dot{x}^{\nu}\right.} \\
& \quad=-\frac{S^{\mu \alpha}}{8 m^{2} c^{2}}\left[\frac{R_{\alpha \nu \beta \sigma} \dot{x}^{\beta}(S \theta \dot{x})^{\sigma}}{2 m c \sqrt{-\dot{x} G \dot{x}}}+S^{\beta \sigma}\left(\nabla R_{\alpha \nu \beta \sigma}\right)\right] \dot{x}^{\nu} .
\end{aligned}
$$

Using this expression, (67) reads (three-dimensional geometry is defined now by $g$; in this case we can not use (51) and (52) to estimate the acceleration)

$$
\frac{d}{\mathrm{~d} \tau}\left[\frac{\dot{x}^{\mu}}{\sqrt{-\dot{x} G \dot{x}}}\right]=\frac{f^{\mu}}{\sqrt{-\dot{x} G \dot{x}}},
$$

where we denoted

$$
\begin{aligned}
f^{\mu} \equiv & \frac{1}{8(m c)^{2}}(T S)^{\mu \alpha} \\
& \cdot\left[\frac{R_{\alpha \nu \beta \sigma} \dot{x}^{\beta}(S \theta \dot{x})^{\sigma}}{\left.2 m c \sqrt{-\dot{x} G \dot{x}}+S^{\beta \sigma}\left(\nabla R_{\alpha \nu \beta \sigma}\right)\right] \dot{x}^{\nu}}\right. \\
& -(\Gamma \dot{x} \dot{x})^{\mu}-\frac{\sqrt{-\dot{x} G \dot{x}}}{4 m c}(T \theta \dot{x})^{\mu} .
\end{aligned}
$$

It will be sufficient to consider static metric $g_{\mu \nu}(\mathbf{x})$ with $g_{0 i}=$ 0 . Then three-dimensional metric and velocity are

$$
\begin{aligned}
\gamma_{i j} & =g_{i j}, \\
v^{i} & =\frac{c}{\sqrt{-g_{00}}} \frac{d x^{i}}{d x^{0}} .
\end{aligned}
$$

Taking $\tau=x^{0}$, the spatial part of (69) with this metric reads

$$
\left(\frac{d t}{d x^{0}}\right)^{-1} \frac{d}{d x^{0}}\left[\frac{v^{i}}{\sqrt{-v G v}}\right]=\frac{f^{i}(v)}{\sqrt{-v G v}}
$$

with $v^{\mu}$ defined in (37), for the case

$$
\begin{aligned}
v^{\mu} & =\left(\frac{c}{\sqrt{-g_{00}}}, \mathbf{v}\right), \\
-v G v & =-v \widetilde{T} v=c^{2}-\mathbf{v} g \mathbf{v}+\frac{(v S \theta v)}{8 m^{2} c^{2}} .
\end{aligned}
$$

In the result, we have presented the equation for trajectory in the form convenient for analysis of acceleration; see (36). Using the definition of three-dimensional covariant derivative (42), we present the derivative on the l.h.s. of (72) as follows:

$$
\begin{gathered}
\frac{d}{d x^{0}}\left[\frac{v^{i}}{\sqrt{-v G v}}\right]=\frac{1}{\sqrt{-v G v}}\left[\mathscr{M}_{k}^{i} \nabla_{0} v^{k}\right. \\
\left.-\widetilde{\Gamma}(\gamma)_{j k}^{i} v^{j} v^{k} \frac{d t}{d x^{0}}+\frac{K v^{i}}{2(-v G v)}\right] .
\end{gathered}
$$

We have denoted

$$
\begin{aligned}
K & =\left(\nabla_{0} G_{\mu \nu}\right) v^{\mu} v^{\nu}-v^{\mu} G_{\mu 0} v^{k} \partial_{k} \ln \left(-g_{00}\right), \\
\mathscr{M}^{i}{ }_{k} & =\delta_{k}^{i}-\frac{v^{i} v^{\mu} G_{\mu k}}{v G v} .
\end{aligned}
$$

The matrix $\mathscr{M}^{i}{ }_{k}$ has the inverse

$$
\begin{aligned}
\widetilde{\mathscr{M}}_{k}^{i} & =\delta^{i}{ }_{k}+\frac{v^{i} v^{\mu} G_{\mu k}}{v^{\sigma} G_{\sigma 0} v^{0}}, \\
\text { then } \widetilde{\mathscr{M}}_{k}^{i} v^{k} & =v^{i} \frac{v G v}{v^{\sigma} G_{\sigma 0} v^{0}} .
\end{aligned}
$$

Combining these equations, we obtain the three-acceleration of our spinning particle

$$
a^{i}=\left(\frac{d t}{d x^{0}}\right)^{-1} \nabla_{0} v^{i}=\widetilde{\mathscr{M}}_{k}^{i}\left[f^{k}+(\widetilde{\Gamma} v)^{k}\right]+\frac{K v^{i}}{2 v^{\sigma} G_{\sigma 0}} .
$$


Finally, using manifest form of $f^{i}$ from (70) we have

$$
\begin{aligned}
a^{i}= & \frac{\widetilde{M}_{k}^{i} \widehat{S}^{k}}{16(m c)^{3} \sqrt{-v G v}}-c^{2} \widetilde{\mathscr{M}}_{k}^{i} \frac{\gamma^{k j} \partial_{j} g_{00}}{2 g_{00}} \\
& -\frac{\sqrt{-v G v}}{4 m c} \widetilde{\mathscr{M}}_{k}^{i}(T \theta v)^{k}+\frac{K v^{i}}{2 v^{\sigma} G_{\sigma 0}} \\
& +\frac{1}{8(m c)^{2}} \widetilde{\mathscr{M}}_{k}^{i}(T S)^{k \alpha} R_{\alpha \nu \beta \sigma ; \lambda} S^{\beta \sigma} v^{v} v^{\lambda}
\end{aligned}
$$

The longitudinal acceleration is obtained by projecting $a^{i}$ on the direction of velocity; that is,

$$
\begin{aligned}
(\mathbf{v} \gamma \mathbf{a})= & \frac{(\mathbf{v} \gamma \widetilde{\mathscr{M}})_{k} \widehat{S}^{k}}{16(m c)^{3} \sqrt{-v G v}}-c^{2}(\mathbf{v} \gamma \widetilde{\mathscr{M}})_{k} \frac{\gamma^{k j} \partial_{j} g_{00}}{2 g_{00}} \\
& -\frac{\sqrt{-v G v}}{4 m c}(\mathbf{v} \gamma \widetilde{M})_{k}(T \theta v)^{k}+\frac{K}{2 v^{\sigma} G_{\sigma 0}}(\mathbf{v} \gamma \mathbf{v}) \\
& +\frac{1}{8(m c)^{2}}(\mathbf{v} \gamma \widetilde{M})_{k}(T S)^{k \alpha} R_{\alpha \nu \beta \sigma ; \lambda} S^{\beta \sigma} v^{v} v^{\lambda}
\end{aligned}
$$

where $\widehat{S}^{k}=(T S)^{k \mu} R_{\mu \nu \alpha \beta} v^{\nu} v^{\alpha}(S \theta v)^{\beta}$. As the speed of the particle closes to the critical velocity, the longitudinal acceleration diverges due to the first term in (79). In resume, assuming that MPTD particle sees the original geometry $g_{\mu \nu}$, we have a theory with unsatisfactory behavior in the ultrarelativistic limit.

Let us consider the second possibility; that is, we take $G_{\mu \nu}$ to construct the three-dimensional geometry (20)-(23). With these definitions we have, by construction, $-\dot{x} G \dot{x}=$ $\left(d t / d x^{0}\right)^{2}\left(c^{2}-(\mathbf{v} \gamma \mathbf{v})\right)$, so the critical speed coincides with the speed of light. In the present case, the expression for threeacceleration can be obtained in closed form for an arbitrary curved background. Taking $\tau=x^{0}$ the spatial part of (69) implies

$$
\left(\frac{d t}{d x^{0}}\right)^{-1} \frac{d}{d x^{0}}\left[\frac{v^{i}}{\sqrt{c^{2}-\mathbf{v} \gamma \mathbf{v}}}\right]=\frac{f^{i}(v)}{\sqrt{c^{2}-\mathbf{v} \gamma \mathbf{v}}}
$$

where, from (70), $f^{i}$ is given by

$$
\begin{aligned}
f^{i} \equiv & \frac{1}{8(m c)^{2}}(T S)^{i \alpha} \\
& \cdot\left[\frac{R_{\alpha \nu \beta \sigma} v^{\beta}(S \theta v)^{\sigma}}{2 m c \sqrt{c^{2}-\mathbf{v} \gamma \mathbf{v}}}+S^{\beta \sigma}\left(\nabla R_{\alpha \nu \beta \sigma}\right)\right] v^{v} \\
& -\Gamma_{\mu \nu}^{i}(G) v^{\mu} v^{l} v-\frac{\sqrt{c^{2}-\mathbf{v} \gamma \mathbf{v}}}{4 m c}(T \theta v)^{i}
\end{aligned}
$$

Equation (80) is of the form (36), so the acceleration is given by (43) and (44) where, for the present case, $\gamma_{i j}=G_{i j}-G_{0 i} G_{0 j} /$ $G_{00}$ :

$$
\begin{aligned}
a^{i} & =\widetilde{M}_{j}^{i}\left[f^{j}+\widetilde{\Gamma}_{k l}^{j}(\gamma) v^{k} v^{l}\right]+\frac{1}{2}\left(\frac{d t}{d x^{0}}\right)^{-1}\left[\left(\mathbf{v} \partial_{0} \gamma \gamma^{-1}\right)^{i}\right. \\
& \left.-\frac{\left(\mathbf{v} \partial_{0} \gamma \mathbf{v}\right)}{c^{2}} v^{i}\right], \\
\mathbf{v} \gamma \mathbf{a} & =\left(1-\frac{\mathbf{v} \gamma \mathbf{v}}{c^{2}}\right)\left[(\mathbf{v} \gamma)_{i}\left[f^{i}(v)+\widetilde{\Gamma}_{k l}^{i}(\gamma) v^{k} v^{l}\right]\right. \\
& \left.+\frac{1}{2}\left(\frac{d t}{d x^{0}}\right)^{-1}\left(\mathbf{v} \partial_{0} \gamma \mathbf{v}\right)\right] .
\end{aligned}
$$

With $f^{i}$ given in (81), the longitudinal acceleration vanishes as $v \rightarrow c$.

Let us resume the results of this section. Assuming that spinning particle probes the three-dimensional spacetime geometry determined by the original metric $g$, we have a theory with unsatisfactory ultrarelativistic limit. First, the critical speed, which the particle can not overcome during its evolution in gravitational field, can be more than the speed of light. The same observation has been made from analysis of MPTD particle in specific metrics [49-53]. Second, the longitudinal acceleration grows up to infinity in the ultrarelativistic limit. Assuming that the particle sees the effective metric $G(S)$ as the space-time metric, we avoided the two problems. But the resulting theory still possesses the problem. The acceleration (82) contains the singularity due to $f^{i} \sim 1 / \sqrt{c^{2}-(\mathbf{v} \gamma \mathbf{v})}$; that is, at $v=c$ the acceleration becomes orthogonal to the velocity, but remains divergent. Besides, due to dependence of effective metric on spin, we arrive at rather unusual picture of the Universe with rainbow geometry (some models of doubly special relativity predict rainbow geometry at Planck scale [54-57]): there is no unique spacetime manifold for the Universe of spinning particles: each particle will probe its own three-dimensional geometry. We conclude that MPTD equations do not seem promising candidate for the description of a relativistic rotating body. It would be interesting to find their generalization with improved behavior in ultrarelativistic regime. This will be achieved within the framework of vector model of spinning particle, which we shall describe in the subsequent sections.

\section{Vector Model of Nonrelativistic Spinning Particle}

The data of some experiments with elementary particles and atoms (Stern-Gerlach experiment, fine structure of hydrogen atom, Zeeman effect) shows that the Schrödinger equation for a one-component wave function is not adequate to describe the behavior of these systems in the presence of an electromagnetic field. This implies a radical modification of the formalism. Besides the position and the momentum, the state of an electron is specified by some discrete numbers, which are eigenvalues of suitably defined operators, called the operators of spin. The mathematical theory of these 
operators is similar to the formalism of angular momentum. So, intuitively, an elementary particle carries an intrinsic angular momentum called spin.

To describe a particle with spin $s=1 / 2$ we introduce the two-component wave function $\Psi_{\alpha}, \alpha=1,2$. The spin operators $\widehat{S}_{i}$ act on $\Psi_{\alpha}$ as $2 \times 2$-matrices and are defined by

$$
\widehat{S}_{i}=\frac{\hbar}{2} \sigma_{i}
$$

where $\sigma_{i}$ stands for the Pauli matrices; they form a basis of the vector space of traceless and Hermitian $2 \times 2$-matrices:

$$
\begin{aligned}
& \sigma_{1}=\left(\begin{array}{ll}
0 & 1 \\
1 & 0
\end{array}\right), \\
& \sigma_{2}=\left(\begin{array}{cc}
0 & -i \\
i & 0
\end{array}\right), \\
& \sigma_{3}=\left(\begin{array}{cc}
1 & 0 \\
0 & -1
\end{array}\right) .
\end{aligned}
$$

Their basic algebraical properties are

$$
\begin{aligned}
\sigma_{i} \sigma_{j}+\sigma_{j} \sigma_{i} & =2 \times \mathbf{1} \delta_{i j}, \\
\sigma_{i} \sigma_{j}-\sigma_{j} \sigma_{i} & \equiv\left[\sigma_{i}, \sigma_{j}\right]=2 i \epsilon_{i j k} \sigma_{k}, \\
\sum_{i} \sigma_{i} \sigma_{i} & =3 \times 1 .
\end{aligned}
$$

Note that the commutators (87) of $\sigma$-matrices are the same for the angular momentum vector. The spin operators, being proportional to the Pauli matrices, have similar properties, in particular

$$
\begin{aligned}
{\left[\widehat{S}_{i}, \widehat{S}_{j}\right] } & =i \hbar \epsilon_{i j k} \widehat{S}_{k}, \\
\widehat{\mathbf{S}}^{2} & =\hbar^{2} s(s+1) \times \mathbf{1}=\frac{3 \hbar^{2}}{4} \mathbf{1} .
\end{aligned}
$$

Consider Coulomb electric and a constant magnetic fields. The electromagnetic potential can be taken in the forms $A_{0}=$ $\alpha / r$ and $\mathbf{A}=(1 / 2)[\mathbf{B} \times \mathbf{r}]$. Then evolution of an electron immersed in this fields is described by the equation

$$
\begin{aligned}
i \hbar & \frac{\partial \Psi}{\partial t}=\left(\frac{1}{2 m}\left(\widehat{\mathbf{p}}-\frac{e}{c} \mathbf{A}\right)^{2}-e A_{0}+\frac{e(g-1)}{2 m^{2} c^{2}} \widehat{\mathbf{S}}[\widehat{\mathbf{p}} \times \mathbf{E}]\right. \\
& \left.-\frac{e g}{2 m c} \mathbf{B} \widehat{\mathbf{S}}\right) \Psi .
\end{aligned}
$$

The first and second terms in the Hamiltonian correspond to the minimal interaction of a point particle with an electromagnetic potential, whereas the last two terms represent interaction of spin with electric and magnetic fields. A numeric factor $g$ is called gyromagnetic ratio of the electron (quantum electrodynamics gives $g=2.002322 \ldots$ due to radiative corrections). The vector $(e g / 2 m c) \widehat{\mathbf{S}}$ is known as magnetic moment of the particle.
The equation is written in the Schrödinger picture; that is, we ascribe time-dependence to the wave function, whereas in semiclassical models we deal with dynamical variables. We recall that the time-dependence can be ascribed to operators using the Heisenberg picture. Passing to the Heisenberg picture, we could write dynamical equations for basic operators of the theory. According to Ehrenfest theorem, expectation values of the operators approximately obey the classical Hamiltonian equations [58].

Equation (91) gives the structure and properties of the energy levels of hydrogen atom in a good agreement with experiment. The fine structure of hydrogen atom fixes the factor $g-1$ in the third term, while Zeeman effect requires the factor $g$ in the last term.

To formulate the problem that we wish to discuss, we recall that quantum mechanics of a spinless particle can be obtained applying the canonical quantization procedure to a classical-mechanics system with the Lagrangian $L=$ $(1 / 2) m x^{2}-U(x)$. To achieve this, we construct a Hamiltonian formulation for the system, then associate with the phasespace variables the operators with commutators resembling the Poisson brackets, and write on this base the Schrödinger equation $i \hbar \dot{\Psi}=\widehat{H} \Psi$.

It is natural to ask whether this ideology can be realized for the spinning particle. Since the quantum-mechanical description of a spin implies the use of three extra operators $\widehat{S}_{i}$, the problem can be formulated as follows. We look for a classical-mechanics system which, besides the position variables $x_{i}$, contains additional degrees of freedom, suitable for the description of a spin: in the Hamiltonian formulation the spin should be described, in the end, by three variables with fixed square (90) and with the classical brackets $\left\{S_{i}, S_{j}\right\}=$ $\epsilon_{i j k} S_{k}$. Then canonical quantization of these variables will yield spin operators with the desired properties (89) and (90). According to this, typical spinning particle model consists of a point on a world-line and some set of variables describing the spin degrees of freedom, which form an inner space attached to that point (there is an elegant formalism developed by Berezin and Marinov $[59,60]$ based on using of anticommuting (Grassmann) variables for the description of spin; we present here another formulation based on commuting variables, without appealing to a rather formal methods of the Grassmann mechanics). In fact, different spinning particles discussed in the literature differ by the choice of the inner space. An exceptional case is the rigid particle [61] which consists of only position variables, but with the action containing higher derivatives. The model yields the Dirac equation [62]; hence it also can be used for description of spin.

It should be noted that (91) is written in the laboratory system, so we do not state that our classical variable $S_{i}$ is a quantity defined in the instantaneous rest frame of the particle.

We intend to construct the spinning particle starting from a suitable variational problem. This is the first task we need to solve, as the formulation of a variational problem in closed form is known only for the case of a phase space equipped with canonical Poisson bracket, say $\left\{\omega_{i}, \pi_{j}\right\}=\delta_{i j}$. The number 
of variables and their algebra are different from the number of spin operators and their commutators, (89). Maybe the most natural way to arrive at the operator algebra (89) is to consider spin as a composite quantity:

$$
\begin{aligned}
S_{i} & =\epsilon_{i j k} \omega_{j} \pi_{k}, \\
\text { or } \mathbf{S} & =\boldsymbol{\omega} \times \boldsymbol{\pi},
\end{aligned}
$$

where $\boldsymbol{\omega}, \boldsymbol{\pi}$ are coordinates of a phase space equipped with canonical Poisson bracket. This immediately induces $\mathrm{SO}(3)$ algebra for $S_{i},\left\{S_{i}(\omega, \pi), S_{j}(\omega, \pi)\right\}_{P B}=\epsilon_{i j k} S_{k}$. Unfortunately, this is not the whole story. First, we need some mechanism which explains why $\mathbf{S}$, not $\boldsymbol{\omega}$ and $\boldsymbol{\pi}$, must be taken for the description of spin degrees of freedom. Second, the basic space is six-dimensional, while the spin manifold is twodimensional (we note that the square of spin operator has fixed value, (90)). To improve this, we look for a variational problem which, besides dynamical equations, implies the constraints

$$
\begin{aligned}
\boldsymbol{\omega} \pi & =0, \\
\pi^{2}-\frac{\alpha}{\omega^{2}} & =0, \quad \text { where } \alpha=\frac{3 \hbar^{2}}{4} .
\end{aligned}
$$

According to Dirac's terminology [63-68], they form the first-class set, so in the model with these constraints the spinsector contains $6-2 \times 2=2$ physical degrees of freedom. Geometrically, the constraints determine four-dimensional $\mathrm{SO}(3)$-invariant surface of the six-dimensional phase space. The constraints imply the fixed value of spin

$$
\mathbf{S}^{2}=\boldsymbol{\omega}^{2} \pi^{2}-(\boldsymbol{\omega} \pi)^{2}=\frac{3 \hbar^{2}}{4} .
$$

The same square of spin follows from the constraints

$$
\begin{aligned}
& \omega^{2}=\alpha^{2}, \\
& \pi^{2}=\beta^{2}, \\
& \omega \pi=0,
\end{aligned}
$$

if we put $\beta^{2}=3 \hbar^{2} / 4 \alpha^{2}$, any $\alpha$. The combination $\pi^{2}-\beta^{2}+$ $\left(\beta^{2} / \alpha^{2}\right)\left(\omega^{2}-\alpha^{2}\right)$ represents the first-class constraint of the set (95). Hence the model with these constraints also has the desired number of degrees of freedom, $6-2-1 \times 2=2$. The equalities (95) determine essentially unique $\mathrm{SO}(3)$-invariant three-dimensional surface of the phase space. The set (93) turns out to be more convenient for generalization to the case of a relativistic spin.

While $\mathbf{S}$ in (92) looks like an angular momentum, the crucial difference is due to the presence of first-class constraints and hence of a local symmetry which we refer as spin-plane symmetry. The latter acts on the basic variables $\boldsymbol{\omega}, \boldsymbol{\pi}$, while leaving invariant the spin variable $\mathbf{S}$. Using analogy with classical electrodynamics, $\boldsymbol{\omega}$ and $\pi$ are similar to four-potential $A^{\mu}$ while $\mathbf{S}$ plays the role of $F^{\mu \nu}$. The coordinates $\boldsymbol{\omega}$ of the "inner space particle" are not physical (observable) quantities. The only observable quantities are the gauge-invariant variables $\mathbf{S}$. So our construction realizes, in a systematic form, the oldest idea about spin as the "hidden angular momentum.”
5.1. Lagrangian and Hamiltonian for the Spin-Sector. As the Lagrangian which implies the constraints (93), we take the expression

$$
L_{\text {spin }}=\frac{\sqrt{\alpha}}{\sqrt{\omega^{2}}} \sqrt{\dot{\boldsymbol{\omega} N} \dot{\boldsymbol{\omega}}}, \quad \alpha=\frac{3 \hbar^{2}}{4},
$$

where $N_{i j}=\delta_{i j}-\left(\omega_{i} \omega_{j} / \omega^{2}\right)$ is the projector on the plane orthogonal to $\boldsymbol{\omega}: N_{i j} \omega^{j}=0, N^{2}=N$. The equivalent forms of the Lagrangian are

$$
L_{\text {spin }}=\frac{\sqrt{\alpha} \sqrt{\omega^{2}(\dot{\boldsymbol{\omega}})^{2}-(\boldsymbol{\omega} \dot{\boldsymbol{\omega}})^{2}}}{\boldsymbol{\omega}^{2}}=\frac{\sqrt{\alpha} \sqrt{\mathbf{S}^{2}}}{\boldsymbol{\omega}^{2}},
$$

where $S_{i}=\epsilon_{i j k} \omega_{j} \dot{\omega}_{k}$. The model is manifestly invariant under global rotations, $\omega_{i}^{\prime}=R_{i j} \omega_{j}$, where $R^{T}=R^{-1}$. There are also two (finite) local symmetries: reparametrizations $t \rightarrow t^{\prime}=$ $\sigma(t) \equiv t+\epsilon(t)$, and the scale transformations $\boldsymbol{\omega} \rightarrow \chi(t) \boldsymbol{\omega}$.

Let us construct the Hamiltonian formulation of the model. Equation for the conjugated momentum reads $\pi=$ $\partial L / \partial \dot{\boldsymbol{\omega}}=\left(\sqrt{\alpha} / \sqrt{\boldsymbol{\omega}^{2}}\right)(N \dot{\boldsymbol{\omega}} / \sqrt{\dot{\boldsymbol{\omega}} N \dot{\boldsymbol{\omega}}})$. This expression immediately implies (93) as the primary constraints. We also note the equality $\pi \dot{\boldsymbol{\omega}}=L$; that is, $H_{0}=\pi \dot{\boldsymbol{\omega}}-L=0$. So the complete Hamiltonian is composed of the primary constraints, $H=$ $v(\boldsymbol{\omega} \boldsymbol{\pi})+v_{1}\left(\boldsymbol{\pi}^{2}-\alpha / \boldsymbol{\omega}^{2}\right)$, and the Hamiltonian action reads

$$
S_{H}=\int d t \pi \dot{\boldsymbol{\omega}}-v(\boldsymbol{\omega} \pi)-v_{1}\left(\pi^{2}-\frac{\alpha}{\boldsymbol{\omega}^{2}}\right) .
$$

There are no of higher-stage constraints in the problem.

Let us write Hamiltonian counterparts of the Lagrangian local symmetries.

(1) Reparametrizations in extended phase space are

$$
\begin{aligned}
t^{\prime} & =\sigma(t), \\
\boldsymbol{\omega}^{\prime} & =\boldsymbol{\omega}, \\
\boldsymbol{\pi}^{\prime} & =\boldsymbol{\pi}, \\
v^{\prime} & =(\dot{\sigma})^{-1} v, \\
v_{1}^{\prime} & =(\dot{\sigma})^{-1} v_{1} .
\end{aligned}
$$

They induce the transformations of dynamical variables

$$
\begin{aligned}
& \boldsymbol{\omega}^{\prime}(\tau)=\boldsymbol{\omega}(\widetilde{\sigma}(\tau)), \\
& \boldsymbol{\pi}^{\prime}(\tau)=\boldsymbol{\pi}(\widetilde{\sigma}(\tau)), \\
& v^{\prime}(\tau)=(\dot{\sigma})^{-1} v(\widetilde{\sigma}(\tau)), \\
& v_{1}^{\prime}(\tau)=(\dot{\sigma})^{-1} v_{1}(\widetilde{\sigma}(\tau)) .
\end{aligned}
$$

Their infinitesimal form reads

$$
\begin{gathered}
\delta \boldsymbol{\omega}=-\epsilon \dot{\boldsymbol{\omega}}, \\
\delta \boldsymbol{\pi}=-\epsilon \dot{\boldsymbol{\pi}}, \\
\delta v=-(\epsilon v), \\
\delta v_{1}=-\left(\epsilon v_{1}\right) .
\end{gathered}
$$


(2) Scale transformations of coordinates are

$$
\begin{aligned}
\tau^{\prime} & =\tau, \\
\boldsymbol{\omega}^{\prime} & =\chi \omega, \\
\pi^{\prime} & =\frac{1}{\chi} \pi, \\
v^{\prime} & =v+\frac{\dot{\chi}}{\chi}, \\
v_{1}^{\prime} & =\chi^{2} v_{1} .
\end{aligned}
$$

Since $\tau$ is not involved, the induced transformations of dynamical variables are the same, for instance, $\boldsymbol{\omega}^{\prime}(\tau)=\chi \boldsymbol{\omega}(\tau)$. Presenting $\chi=1+\gamma$, infinitesimal transformations of dynamical variables read

$$
\begin{aligned}
\delta \boldsymbol{\omega} & =\gamma \boldsymbol{\omega}, \\
\delta \boldsymbol{\pi} & =-\gamma \boldsymbol{\pi}, \\
\delta v & =\dot{\gamma}, \\
\delta v_{1} & =2 \gamma v_{1} .
\end{aligned}
$$

Besides the constraints (93), the variational problem (98) implies the Hamiltonian equations

$$
\begin{aligned}
& \dot{\omega}=\rho \omega^{2} \pi+v \omega, \\
& \dot{\pi}=-\rho \pi^{2} \omega-v \pi .
\end{aligned}
$$

To make the system more symmetric, we have introduced the variable $\rho=2 v_{1} / \omega^{2}$ instead of $v_{1}$.

According to general formalism of constrained systems [63-66], neither the dynamical equations nor the constraints determine the variables $v$ and $\rho$. They enter as arbitrary functions of time into general solution for the variables $\boldsymbol{\omega}$ and $\boldsymbol{\pi}$, making completely undetermined their dynamics. Indeed, for any given functions $v(t)$ and $\rho(t)$, the equations represent a normal system for determining $\boldsymbol{\omega}$ and $\boldsymbol{\pi}$. Its general solution is

$$
\begin{aligned}
\boldsymbol{\omega} & =e^{\int_{0}^{t} v d \tau}\left[\mathbf{b} \cos \left(\sqrt{\alpha} \int_{0}^{t} \rho d \tau\right)\right. \\
& \left.+\mathbf{c} \sin \left(\sqrt{\alpha} \int_{0}^{t} \rho d \tau\right)\right], \\
\boldsymbol{\pi} & =e^{-\int_{0}^{t} v d \tau\left[-\mathbf{b} \sin \left(\sqrt{\alpha} \int_{0}^{t} \rho d \tau\right)\right.} \\
& \left.+\operatorname{cocos}\left(\sqrt{\alpha} \int_{0}^{t} \rho d \tau\right)\right],
\end{aligned}
$$

where the integration constants $\mathbf{b}$ and $\mathbf{c}$ are subject to the conditions

$$
\begin{aligned}
& \mathbf{b} \mathbf{c}=0, \\
& \mathbf{b}^{2}=\mathbf{c}^{2}=\sqrt{\alpha} .
\end{aligned}
$$

This implies $\boldsymbol{\omega}^{2}=\sqrt{\alpha} e^{2 \int_{0}^{t} v d \tau}$ and $\pi^{2}=\sqrt{\alpha} e^{-2 \int_{0}^{t} v d \tau}$. According to these expressions, the pair of orthogonal vectors $\boldsymbol{\omega}$ and $\pi$ rotate in their own plane (or, equivalently, in the plane determined by $\mathbf{b}$ and $\mathbf{c}$ ) with the variable angular velocity prescribed by the function $\rho(t)$. The function $v(\tau)$ determines the variation of their magnitudes. Choosing the functions $v$ and $\rho$ suitably, we can make the point with radius-vector $\boldsymbol{\omega}$ move along any prescribed line!

We point out that the two-parametric ambiguity is in correspondence with the invariance of the action (98) under the two local symmetries described above. Summing up, all the basic variables of our model are unobservable quantities.

The spin-vector (note that this coincides with $\mathbf{S}$ which appeared in (97)) $\mathbf{S}=\boldsymbol{\omega} \times \boldsymbol{\pi}$ has unambiguous evolution

$$
\dot{\mathbf{S}}=0 \text {. }
$$

Note also that it is invariant of the local symmetries. Hence the spin-vector is a candidate for an observable quantity. In interacting theory $\mathbf{S}$ will precess under the torque exercised by a magnetic field; see below. Due to (93), the coordinates $S_{i}$ obey (94).

5.2. Spin Fiber Bundle and Spin-Plane Local Symmetry. The passage from initial variables $\boldsymbol{\omega}$ and $\boldsymbol{\pi}$ to the observables $\boldsymbol{S}$ is not a change of variables and acquires a natural interpretation in the geometric terms. It should be noted that basic notions of the theory of constrained systems have their analogs in differential geometry. Second-class constraints imply that all true trajectories lie on a submanifold of the initial phasespace. The Dirac bracket, constructed on the base of secondclass constraints, induces canonical symplectic structure on the submanifold. If the first-class constraints (equivalently, the local symmetries) are presented in the model, a part of variables have ambiguous evolution. This also can be translated into the geometric language: due to the ambiguity, the submanifold should be endowed with a natural structure of a fiber bundle. Physical variables are (functions of) the coordinates which parameterize the base of the fiber bundle. Let us describe how all this looks like in our model.

Consider six-dimensional phase space equipped with canonical Poisson bracket

$$
\mathbb{R}^{6}=\left\{\omega_{i}, \pi_{j} ;\left\{\omega_{i}, \pi_{j}\right\}_{\mathrm{PB}}=\delta_{i j}\right\},
$$

and three-dimensional spin space $\mathbb{R}^{3}=\left\{S_{i}\right\}$ with the coordinates $S_{i}$. Define the map

$$
\begin{gathered}
f: \mathbb{R}^{6} \longrightarrow \mathbb{R}^{3}, \\
f:\left(\omega_{i}, \pi_{j}\right) \longrightarrow S_{i}=\epsilon_{i j k} \omega_{j} \pi_{k}, \\
\text { or } \mathbf{S}=\boldsymbol{\omega} \times \boldsymbol{\pi}, \\
\operatorname{rank} \frac{\partial\left(S_{i}\right)}{\partial\left(\omega_{j}, \pi_{k}\right)}=3 .
\end{gathered}
$$


Poisson bracket on $\mathbb{R}^{6}$ together with the map induces $\mathrm{SO}(3)$ Lie-Poisson bracket on $\mathbb{R}^{3}$

$$
\begin{aligned}
\left\{S_{i}, S_{j}\right\} & \equiv\left\{S_{i}(\omega, \pi), S_{j}(\omega, \pi)\right\}_{\mathrm{PB}}, \\
\text { then }\left\{S_{i}, S_{j}\right\} & =\epsilon_{i j k} S_{k} .
\end{aligned}
$$

As we saw above, all the trajectories $\boldsymbol{\omega}(t), \boldsymbol{\pi}(t)$ lie on $\mathrm{SO}(3)$ invariant surface of $\mathbb{R}^{6}$ determined by the constraints

$$
\mathbb{W}^{4}=\left\{\omega \pi=0, \pi^{2}-\frac{\alpha}{\omega^{2}}=0\right\}
$$

that is, $\boldsymbol{\omega}$ and $\boldsymbol{\pi}$ represent a pair of orthogonal vectors with their ends attached to the hyperbole $y=\alpha / x$.

When $(\boldsymbol{\omega}, \boldsymbol{\pi}) \in \mathbb{T}^{4}$, we have $\mathbf{S}^{2}=\boldsymbol{\omega}^{2} \boldsymbol{\pi}^{2}-(\boldsymbol{\omega} \pi)^{2}=\alpha$. So, $f$ maps the manifold $\mathbb{T}^{4}$ onto two-dimensional sphere (spinsurface) of the radius $\sqrt{\alpha}, f\left(\mathbb{T}^{3}\right)=\mathbb{S}^{2}$.

Denote $\mathbb{F}_{S}^{2} \in \mathbb{T}^{4}$ preimage of a point $\mathbf{S} \in \mathbb{S}^{2}, \mathbb{F}_{S}^{2}=$ $f^{-1}(\mathbf{S})$. Let $(\boldsymbol{\omega}, \boldsymbol{\pi}) \in \mathbb{F}_{S}^{2}$. Then the two-dimensional manifold $\mathbb{F}_{S}^{2}$ contains all pairs $(\chi \boldsymbol{\omega},(1 / \chi) \pi), \chi \in \mathbb{R}^{+}$, as well as the pairs obtained by rotation of these $(\chi \boldsymbol{\omega},(1 / \chi) \pi)$ in the plane of vectors $(\boldsymbol{\omega}, \boldsymbol{\pi})$. So elements of $\mathbb{F}_{S}^{2}$ are related by twoparametric transformations

$$
\begin{aligned}
& \boldsymbol{\omega}^{\prime}=\chi \omega, \\
& \pi^{\prime}=-\frac{1}{\chi} \pi, \\
& \omega^{\prime}=\omega \cos \phi+\pi \frac{|\omega|}{|\pi|} \sin \phi, \\
& \pi^{\prime}=-\omega \frac{|\pi|}{|\omega|} \sin \phi+\pi \cos \phi .
\end{aligned}
$$

In the result, the manifold $\mathbb{T}^{4}$ acquires a natural structure of fiber bundle

$$
\mathbb{T}^{4}=\left(\mathbb{S}^{2}, \mathbb{F}^{2}, f\right)
$$

with base $\mathbb{S}^{2}$, standard fiber $\mathbb{F}^{2}$, projection map $f$, and structure group given by the transformations (112) and (113). The adjusted with the structure of the fiber bundle local coordinates are $\chi, \phi$, and two coordinates of the vector $\mathbf{S}$. By construction, the structure group transformations leave inert points of the base, $\delta S_{i}=0$.

Let us discuss the relationship between the structure group and local symmetries of the Hamiltonian action (98). The structure transformation (112) can be identified with the scale transformation (102). Concerning the transformation (113), let us apply it to the action (98). Inserting $\boldsymbol{\omega}^{\prime}$ and $\boldsymbol{\pi}^{\prime}$ into the action and disregarding the total derivative, we obtain the expression

$$
\begin{aligned}
S_{H}\left[q^{\prime}\right]= & \int d \tau \pi \dot{\boldsymbol{\omega}}-\boldsymbol{\omega} \boldsymbol{\pi}\left[v^{\prime} \cos 2 \phi+B-v_{1}^{\prime} A\right] \\
& -\left(\boldsymbol{\pi}^{2}-\frac{\alpha}{\boldsymbol{\omega}^{2}}\right)\left[v_{1}^{\prime}-C\right],
\end{aligned}
$$

where

$$
\begin{aligned}
& A=\frac{|\boldsymbol{\pi}|}{|\boldsymbol{\omega}|}\left(1-\frac{\alpha}{\boldsymbol{\omega}^{2} \pi^{2}+(\boldsymbol{\omega} \pi)|\boldsymbol{\omega}||\boldsymbol{\pi}| \sin 2 \phi}\right) \sin 2 \phi \\
& B=\left(\frac{|\boldsymbol{\omega}|}{|\boldsymbol{\omega}|}-\frac{|\boldsymbol{\pi}|}{|\boldsymbol{\pi}|}\right) \sin ^{2} \phi \\
& C=\frac{\dot{\phi} \boldsymbol{\omega}^{2}}{|\boldsymbol{\omega}||\boldsymbol{\pi}|+\sqrt{\alpha}} .
\end{aligned}
$$

The action does not change, $S_{H}\left[q^{\prime}\right]=S_{H}[q]$, if we adopt the following transformation law for $v$ and $v_{1}$ :

$$
\begin{aligned}
& v^{\prime}=\frac{v-B+A\left(v_{1}+C\right)}{\cos 2 \phi}, \\
& v_{1}^{\prime}=v_{1}+C .
\end{aligned}
$$

Hence we have found one more local symmetry of the action. Its infinitesimal form reads

$$
\begin{aligned}
\delta \boldsymbol{\omega} & =\phi \frac{|\boldsymbol{\omega}|}{|\boldsymbol{\pi}|} \boldsymbol{\pi}, \\
\delta \boldsymbol{\pi} & =-\phi \frac{|\boldsymbol{\pi}|}{|\boldsymbol{\omega}|} \boldsymbol{\omega}, \\
\delta v & =\frac{2 \phi v_{1}}{|\boldsymbol{\omega}||\boldsymbol{\pi}|}\left(\boldsymbol{\pi}^{2}-\frac{\alpha}{\boldsymbol{\omega}^{2}}\right), \\
\delta v_{1} & =\frac{\dot{\phi} \boldsymbol{\omega}^{2}}{|\boldsymbol{\omega}||\boldsymbol{\pi}|+\sqrt{\alpha}} .
\end{aligned}
$$

The three infinitesimal symmetries (101), (103), and (118) are not independent of the subspace of solutions to equations of motion. To see this, we note that the following infinitesimal transformation:

$$
\begin{aligned}
\delta_{\epsilon}+\delta_{\gamma(\epsilon)}+\delta_{\phi(\epsilon)}, & \\
& \text { where } \gamma(\epsilon)=\epsilon \nu, \phi(\epsilon)=2 v_{1} \frac{|\boldsymbol{\omega}|}{|\boldsymbol{\pi}|} \epsilon,
\end{aligned}
$$

being applied to any variable, turns out to be proportional to equations of motion. For instance, $\left[\delta_{\epsilon}+\delta_{\gamma(\epsilon)}+\delta_{\phi(\epsilon)}\right] \pi=$ $-\epsilon\left(\delta S_{H} / \delta \pi\right)-\left(2 \epsilon v_{1} \boldsymbol{\omega} / \boldsymbol{\omega}^{2}\right)\left(\delta S_{H} / \delta v_{1}\right)$. The on-shell symmetries are considered as trivial symmetries; see $[69,70]$. Hence on the subspace of solutions the infinitesimal reparametrization can be identified with a special transformation of the structure group

$$
\delta_{\epsilon}=-\delta_{\gamma(\epsilon)}-\delta_{\phi(\epsilon)}
$$

In the result, the number of infinitesimal symmetries coincides with the number of primary first-class constraints. Summing up, in the passage from geometric to dynamical realization, the transformations of structure group of the spin fiber bundle act independently at each instance of time and turn into the local symmetries of Hamiltonian action.

Equivalent Formulations. Let us consider a slightly different Lagrangian

$$
L_{\text {spin }}=\frac{1}{2} \dot{\boldsymbol{\omega}} N \dot{\boldsymbol{\omega}}+\frac{\alpha}{2 \omega^{2}} .
$$


The conjugated momentum $\pi=N \dot{\boldsymbol{\omega}}$ implies only one primary constraint $\boldsymbol{\omega} \boldsymbol{\pi}=0$; then the complete Hamiltonian reads

$$
H=\frac{1}{2}\left(\pi^{2}-\frac{\alpha}{\omega^{2}}\right)+v(\boldsymbol{\omega} \pi)
$$

Computing $(d / d t)(\boldsymbol{\omega} \pi)=\{\boldsymbol{\omega} \boldsymbol{\pi}, H\}$, we obtain $\boldsymbol{\pi}^{2}-\left(\alpha / \boldsymbol{\omega}^{2}\right)=0$ as the secondary constraint. Hence the Lagrangian implies an equivalent formulation.

As the Lagrangian which implies the constraints (95), we could take the expression $L_{\text {spin }}=(1 / 2 g) \dot{\boldsymbol{\omega}}^{2}+(1 / 2) g \beta^{2}-$ $(1 / 2) \lambda\left(\omega^{2}-\alpha^{2}\right)$. We note that in this model $\beta^{2}=3 \hbar^{2} / 4 \alpha^{2}$, while $\alpha$ is any given number. Variation with respect to auxiliary variables $g(t)$ and $\lambda(t)$ gives the equations $\dot{\boldsymbol{\omega}}^{2}=g^{2} \beta^{2}$ and $\boldsymbol{\omega}^{2}=\alpha^{2}$; the latter implies $\dot{\boldsymbol{\omega}} \boldsymbol{\omega}=0$. In the Hamiltonian formulation these equations turn into the desired constraints. We can integrate out the variable $g$, presenting the Lagrangian in a more compact form

$$
L_{\text {spin }}=\beta \sqrt{\dot{\omega}^{2}}-\frac{1}{2} \lambda\left(\omega^{2}-\alpha^{2}\right)
$$

This also gives the desired constraints. The last term represents kinematic (velocity-independent) constraint. So, we might follow the known classical-mechanics prescription and exclude $\lambda$ as well. But this would lead to loss of the manifest rotational invariance of the formalism. The spin fiber bundle corresponding to this formulation turns out to be the group manifold $\mathrm{SO}(3)$; see [71] for details.

5.3. Canonical Quantization and Pauli Equation. To test our formulation, we show that our spinning particle yields the Pauli equation in a stationary magnetic field with the vector potential A. Consider the action

$$
\begin{aligned}
S & =\int d t\left[\frac{m}{2} \dot{\mathbf{x}}^{2}+\frac{e}{c} \mathbf{A} \dot{\mathbf{x}}+\frac{\sqrt{\alpha}}{\sqrt{\boldsymbol{\omega}^{2}}} \sqrt{D \boldsymbol{\omega} N D \boldsymbol{\omega}}\right], \\
D \omega_{i} & =\dot{\omega}_{i}-\frac{g e}{2 m c} \epsilon_{i j k} \omega_{j} B_{k} .
\end{aligned}
$$

The configuration-space variables are $x_{i}(t)$ and $\omega_{i}(t)$. Here $x_{i}$ represents the spatial coordinates of the particle with the mass $m$, charge $e$, and gyromagnetic ratio $g$. In our classical model $g$ appeared as a coupling constant of $\boldsymbol{\omega}$ with the magnetic field $\mathbf{B}=\boldsymbol{\nabla} \times \mathbf{A}$ in the last term of (125). At the end, it produces the Pauli term in the quantum-mechanical Hamiltonian.

Let us construct Hamiltonian formulation for the model. Equations for the conjugated momenta $p_{i}$ and $\pi_{i}$ read

$$
\begin{aligned}
& \mathbf{p}=m \dot{\mathbf{x}}+\frac{e}{c} \mathbf{A}, \Longrightarrow \\
& \dot{\mathbf{x}}=\frac{1}{m}\left(\mathbf{p}-\frac{e}{c} \mathbf{A}\right), \\
& \boldsymbol{\pi}=\frac{\sqrt{\alpha}}{\sqrt{\boldsymbol{\omega}^{2}}} \frac{N D \boldsymbol{\omega}}{\sqrt{D \boldsymbol{\omega N D \boldsymbol { \omega }}}} .
\end{aligned}
$$

Eq. (127) implies the primary constraints $\boldsymbol{\omega} \pi=0$ and $\boldsymbol{\pi}^{2}-$ $\alpha / \boldsymbol{\omega}^{2}=0$. The complete Hamiltonian, $H=P \dot{Q}-L+v_{a} \Phi_{a}, Q=$ $(\mathbf{x}, \boldsymbol{\omega}), P=(\mathbf{p}, \boldsymbol{\pi})$, reads

$$
\begin{aligned}
H= & \frac{1}{2 m}\left(\mathbf{p}-\frac{e}{c} \mathbf{A}\right)^{2}-e A_{0}-\frac{g e}{2 m c}(\mathbf{B S})+v(\boldsymbol{\omega} \boldsymbol{\pi}) \\
& +v_{1}\left(\boldsymbol{\pi}^{2}-\frac{\alpha}{\boldsymbol{\omega}^{2}}\right) .
\end{aligned}
$$

There are no of higher-stage constraints in the formulation. Besides the constraints, the Hamiltonian (128) implies the dynamical equations

$$
\begin{aligned}
& \dot{x}_{i}=\frac{1}{m}\left(p_{i}-\frac{e}{c} A_{i}\right) \\
& \dot{p}_{i}=\frac{e}{c} \dot{x}_{j} \partial_{i} A_{j}+\frac{g e}{2 m c} S_{j} \partial_{i} B_{j}, \\
& \dot{\omega}_{i}=v \omega_{i}+2 v_{1} \pi_{i}+\frac{g e}{2 m c} \epsilon_{i j k} \omega_{j} B_{k}, \\
& \dot{\pi}_{i}=-v \pi_{i}-2 v_{1} \frac{\pi^{2}}{\omega^{2}} \omega_{i}+\frac{g e}{2 m c} \epsilon_{i j k} \pi_{j} B_{k} .
\end{aligned}
$$

As a consequence of these equations, the spin-vector $S_{i}=$ $\epsilon_{i j k} \omega_{j} \pi_{k}$ has unambiguous evolution

$$
\dot{S}_{i}=\frac{g e}{2 m c} \epsilon_{i j k} S_{j} B_{k} .
$$

This is the classical equation for precession of spin in an external magnetic field. Due to (95), the coordinates $S_{i}$ obey (94). Equations (129) imply the second-order equation for $x_{i}$

$$
m \ddot{x}_{i}=\frac{e}{c} \epsilon_{i j k} \dot{x}_{j} B_{k}+\frac{g e}{2 m c} S_{k} \partial_{i} B_{k}
$$

Note that, in the absence of interaction, the spinning particle does not experience a self-acceleration. The last term gives nonvanishing contribution into the trajectory in inhomogeneous field and can be used for semiclassical description of Stern-Gerlach experiment. Since $S^{2} \sim \hbar^{2}$, the $S$-term disappears from (132) at the classical limit $\hbar \rightarrow 0$. Then (132) reproduces the classical motion of a charged particle subject to the Lorentz force.

5.3.1. Precession of Spin. Let us denote $-(g e / 2 m c) \mathbf{B}=\boldsymbol{\omega}_{p}$; then (131) reads

$$
\dot{\mathbf{S}}=\boldsymbol{\omega}_{p} \times \mathbf{S}
$$

The vector $\dot{\mathbf{S}}$ is orthogonal to the plane of $\boldsymbol{\omega}_{p}$ and $\mathbf{S}$ at any instant. Besides, contracting (133) with $\mathbf{S}$ we see that magnitude of spin does not change, $S^{2}=$ const. In the result, the end point of $\mathbf{S}$ rotates around the axis $\boldsymbol{\omega}_{p}$. Let $\mathbf{S}(0)=\mathbf{S}_{0}$ be the initial position of spin. We present this vector as a sum of longitudinal and transversal parts with respect to $\omega_{p}$, $\mathbf{S}_{0}=\mathbf{S}_{0 \perp}+\mathbf{S}_{0 \|}$. Then for the constant vector $\boldsymbol{\omega}_{p}$, the general solution to (133) is

$$
\mathbf{S}=\mathbf{S}_{0 \|}+\left|\mathbf{S}_{0 \perp}\right|\left(\mathbf{e}_{1} \cos \left|\boldsymbol{\omega}_{p}\right| t+\mathbf{e}_{2} \sin \left|\boldsymbol{\omega}_{p}\right| t\right) .
$$


Hence the magnitude of vector $\omega_{p}$ from (133) is just the frequency of precession. Equation of trajectory (132) in the constant magnetic field is $\dot{\mathbf{v}}=(2 / g)\left[\boldsymbol{\omega}_{p} \times \mathbf{v}\right]$; that is, particle's velocity precesses with the frequency $(2 / g) \omega_{p}$. For a particle with classical gyromagnetic ratio $g=2$, the two frequencies coincide and the angle between velocity and spin preserves during the evolution. For the anomalous magnetic moment, $g \neq 2$, the frequencies are different. The spin precession relative to the velocity is used in a cyclotron experiments for measurement of anomalous magnetic moment $[18,19]$.

5.3.2. Canonical Quantization. We quantize only the physical variables $x_{i}, p_{i}, S_{i}$. Their classical brackets are

$$
\begin{aligned}
& \left\{x_{i}, p_{j}\right\}=\delta_{i j}, \\
& \left\{S_{i}, S_{j}\right\}=\epsilon_{i j k} S_{k} .
\end{aligned}
$$

As the last two terms in (128) do not contribute into equations of motion for the physical variables, we omit them. This gives the physical Hamiltonian

$$
H=\frac{1}{2 m}\left(\mathbf{p}-\frac{e}{c} \mathbf{A}\right)^{2}-\frac{g e}{2 m c} \mathbf{B S} .
$$

The first equation from (135) implies the standard quantization of the variables $x$ and $p$; we take $\widehat{x}_{i}=x_{i}, \widehat{p}_{i}=-i \hbar \partial_{i}$. According to the second equation from (135), we look for the wave function space which is a representation of the group $\mathrm{SO}(3)$. Finite-dimensional irreducible representations of the group are numbered by spin $s$, which is related to the values of Casimir operator as follows: $S^{2} \sim s(s+1)$. Then (94) fixes the spin $s=1 / 2$, and $S_{i}$ must be quantized by $\widehat{S}_{i}=(\hbar / 2) \sigma_{i}$. The operators act on the spinor space of two-component complex columns $\Psi$. Quantum Hamiltonian is obtained from (136) replacing classical variables by the operators. This immediately yields the Pauli equation, that is, (91) with $\mathbf{E}=0$.

\section{Why Do We Need a Semiclassical Model of Relativistic Spin?}

6.1. Dirac Equation. We expect that a semiclassical relativistic model of spin should be closely related to the Dirac equation normally used to describe the relativistic spin in quantum theory. The consistent description of relativistic spin is achieved in quantum electrodynamics, where the Dirac equation is considered as a quantum field theory equation. But it also admits a quantum-mechanical interpretation and thus represents an example of relativistic quantum mechanics. This is of interest on various reasons. In particular, namely, being considered as a quantum-mechanical equation, the Dirac equation gives the correct energy levels of hydrogen atom. As we saw in Section 5.1, dynamical equations for expectation values of operators in quantum mechanics should resemble the Hamiltonian equations of the corresponding classical system. Let us discuss these equations in the Dirac theory.
Under the infinitesimal Lorentz transformation $\delta x^{\mu}=$ $\omega^{\mu}{ }_{\nu} x^{\nu}$, the Dirac spinor $\Psi=\left(\Psi_{1}, \Psi_{2}, \Psi_{3}, \Psi_{4}\right)$ transforms as follows:

$$
\delta \Psi=-\frac{i}{4} \omega_{\mu \nu} \gamma^{\mu \nu} \Psi
$$

where

$$
\gamma^{\mu \nu} \equiv\left[\gamma^{\mu}, \gamma^{\nu}\right]=\frac{i}{2}\left(\gamma^{\mu} \gamma^{\nu}-\gamma^{\nu} \gamma^{\mu}\right)
$$

and the $4 \times 4 \gamma$-matrices can be composed from $\sigma$-matrices of Pauli

$$
\begin{aligned}
\gamma^{0} & =\left(\begin{array}{cc}
1 & 0 \\
0 & -1
\end{array}\right), \\
\gamma^{i} & =\left(\begin{array}{cc}
0 & \sigma^{i} \\
-\sigma^{i} & 0
\end{array}\right) .
\end{aligned}
$$

We use the representation with Hermitian $\gamma^{0}$ and antiHermitian $\gamma^{i}$. The matrices do not commute with each other, and the basic formula for their permutation is as follows:

$$
\left[\gamma^{\mu}, \gamma^{\nu}\right]_{+} \equiv \gamma^{\mu} \gamma^{\nu}+\gamma^{\nu} \gamma^{\mu}=-2 \eta^{\mu \nu}
$$

The Dirac equation in an external four-potential $A_{\mu}$

$$
\left[\gamma^{\mu}\left(\widehat{p}_{\mu}-\frac{e}{c} A_{\mu}\right)+m c\right] \Psi=0, \quad \text { where } \widehat{p}_{\mu}=-i \hbar \partial_{\mu}
$$

turns out to be covariant under the transformation (137). Applying the operator $\gamma^{\mu}\left(\widehat{p}_{\mu}-(e / c) A_{\mu}\right)-m c$ to (141), we see that the Dirac equation implies the Klein-Gordon equation with nonminimal interaction

$$
\left[\left(\widehat{p}^{\mu}-\frac{e}{c} A^{\mu}\right)^{2}-\frac{e \hbar}{2 c} F_{\mu \nu} \gamma^{\mu \nu}+m^{2} c^{2}\right] \Psi=0,
$$

where $F_{\mu \nu}=\partial_{\mu} A_{\nu}-\partial_{\nu} A_{\mu}$.

For the latter use, let us analyze commutators of the matrices involved. The commutators of $\gamma$-matrices can not be presented through themselves, but produce $\gamma^{\mu \nu}$-matrices as they are written in (138). The set $\gamma^{\mu}, \gamma^{\mu \nu}$ forms a closed algebra

$$
\begin{aligned}
& {\left[\gamma^{\mu}, \gamma^{\nu}\right]=-2 i \gamma^{\mu \nu},} \\
& {\left[\gamma^{\mu \nu}, \gamma^{\alpha}\right]=2 i\left(\eta^{\mu \alpha} \gamma^{\nu}-\eta^{\nu \alpha} \gamma^{\mu}\right),} \\
& {\left[\gamma^{\mu \nu}, \gamma^{\alpha \beta}\right]} \\
& \quad=2 i\left(\eta^{\mu \alpha} \gamma^{\nu \beta}-\eta^{\mu \beta} \gamma^{\nu \alpha}-\eta^{\nu \alpha} \gamma^{\mu \beta}+\eta^{\nu \beta} \gamma^{\mu \alpha}\right) .
\end{aligned}
$$

As it was tacitly implied in (137), $\gamma^{\mu \nu}$-matrices obey $\mathrm{SO}(1,3)$ algebra of Lorentz generators. The complete algebra (143) can be identified with the five-dimensional Lorentz algebra $\mathrm{SO}(2,3)$ with generators $J^{A B}, A, B=(\mu, 5)=(0,1,2,3,5)$, and with the metric $\eta^{A B}=(-,+,+,+,-)$ :

$$
\begin{aligned}
& {\left[J^{A B}, J^{C D}\right]} \\
& \quad=2 i\left(\eta^{A C} J^{B D}-\eta^{A D} J^{B C}-\eta^{B C} J^{A D}+\eta^{B D} J^{A C}\right),
\end{aligned}
$$


assuming $\gamma^{\mu} \equiv J^{5 \mu}, \gamma^{\mu \nu} \equiv J^{\mu \nu}$. Vector model of spinning particle with $\mathrm{SO}(2,3)$ covariant spin space has been constructed in [72].

6.2. Observer-Independent Probability. $\Psi$ can be used to construct the adjoint spinor $\bar{\Psi}=\Psi^{\dagger} \gamma^{0}$ with the transformation law $\delta \bar{\Psi}=(i / 4) \bar{\Psi} \gamma^{\mu \nu} \omega_{\mu \nu}$. Then $\bar{\Psi} \Psi$ is a scalar and $\bar{\Psi} \gamma^{\mu} \Psi$ is a vector (with the factor -(i/4) in (137) and with the standard transformation law for a vector, $\delta v_{\mu}=\epsilon_{\mu}{ }^{v} v_{v}$, the function $v_{\mu} \bar{\Psi} \gamma^{\mu} \Psi$ is a scalar function) and so on. The vector turns out to be a conserved current, that is,

$$
\partial_{\mu}\left(\bar{\Psi} \gamma^{\mu} \Psi\right)=0
$$

on solutions to the Dirac equation. The time-component of the vector is $\Psi^{\dagger} \Psi$. Assuming that symbols $x^{i}$ represent the position of a particle, the quantity

$$
P(t)=\Psi^{\dagger} \Psi d^{3} x
$$

is identified with relativistic-invariant probability to find a particle in the infinitesimal volume $d^{3} x$ at the instant $t=$ $x^{0} / c$. To confirm this interpretation, we first note that the probability density $\Psi^{\dagger} \Psi$ is a positive function. Second, due to the continuity equation (145), integral of the density over all space does not depend on time: $\left(d / d x^{0}\right) \int_{V} d^{3} x \Psi^{\dagger} \Psi=$ $\int_{V} d^{3} x \partial_{0}\left(\bar{\Psi} \gamma^{0} \Psi\right)=-\int_{V} d^{3} x \partial_{i}\left(\bar{\Psi} \gamma^{i} \Psi\right)=-\int_{\partial V} d \Omega_{i}\left(\bar{\Psi} \gamma^{i} \Psi\right)=0$ for the solutions $\Psi$ that vanish on spatial infinity. Third, $P$ coincides with the manifestly Lorentz-invariant quantity

$$
-\frac{1}{6} \epsilon_{\mu \nu \alpha \beta}\left(\bar{\Psi} \gamma^{\mu} \Psi\right) d x^{\nu} d x^{\alpha} d x^{\beta}
$$

when it computed over equal-time surface $x^{0}=$ const of Minkowski space. This implies an observer-independence of the probability $P$ : all inertial observers, when they compute $P$ using their coordinates, will compute the same number (147).

However, it is well known that, adopting the quantummechanical interpretation, we arrive at a rather strange and controversial picture. We outline here the results of analysis on the applicability of quantum-mechanical treatment to the free Dirac equation made by Schrödinger (for an electron interacting with electromagnetic field this analysis has been repeated by Feynman in [73]) in [74]. We multiply the Dirac equation on $\gamma^{0}$, representing it in the Schrödinger-like form

$$
\begin{aligned}
i \hbar \partial_{t} \Psi & =\widehat{H} \Psi, \\
\widehat{H} & =c \alpha^{i} \widehat{p}_{i}+m c^{2} \beta,
\end{aligned}
$$

where $\alpha^{i}=\gamma^{0} \gamma^{i}$ and $\beta=\gamma^{0}$ are Dirac matrices. Then $\widehat{H}$ may be interpreted as the Hamiltonian. Passing from the Schrödinger to Heisenberg picture, the time derivative of an operator $a$ is $i \hbar \dot{a}=[a, H]$, and for the expectation values of basic operators of the Dirac theory we obtain the equations

$$
\begin{aligned}
\dot{x}_{i} & =c \alpha_{i}, \\
\dot{p}_{i} & =0, \\
i \hbar \dot{\alpha}_{i} & =2\left(c p_{i}-H \alpha_{i}\right), \\
i \hbar \dot{\beta} & =-2 c \alpha_{i} p_{i} \beta+m c^{2} .
\end{aligned}
$$

Some properties of the equations are in order.

(1) The Wrong Balance of the Number of Degrees of Freedom. The first equation in (149) implies that the operator $c \alpha^{i}$ represents the velocity of the particle. Then physical meaning of the operator $p^{i}$ becomes rather obscure in the classical limit.

(2) Zitterbewegung. Equations (149) can be solved, with the result for $x^{i}(t)$ being $x^{i}=a^{i}+d p^{i} t+c^{i} \exp (-(2 i H / \hbar) t)$. The first and second terms are expected and describe a motion along the straight line. The last term on the r.h.s. of this equation states that the free electron experiences rapid oscillations with higher frequency $2 H / \hbar \sim 2 m c^{2} / \hbar$.

(3) Velocity of an Electron. Since the velocity operator $c \alpha^{i}$ has eigenvalues $\pm c$, we conclude that a measurement of a component of the velocity of a free electron is certain to lead to the result $\pm c$.

(4) Operator of Relativistic Spin. We expect that in the Dirac theory can be constructed the relativistic generalization of the spin operator (84). The question on the definition of a conventional spin operator has been raised a long time ago $[75,76]$ and is under discussion up to date [77-79].

Many people noticed that in the Dirac theory it is possible to construct other operators that obey reasonable equations; see $[16,80]$. Presenting these equations, Feynman accompanied them with the following comment (see p. 48 in [73]): "the following relations may be verified as true but their meaning is not yet completely understood, if at all..."

In view of all this, it seem desirable to construct a semiclassical model of spin that will be as close as possible to the Dirac equation. By this we mean the model which, being quantized, yields the Dirac equation. In the following sections, we will see how the vector model clarifies the issues discussed above. In a few words, this can be resumed as follows. As we already saw above, the vector model is necessarily invariant under the spin-plane local symmetry which determines its physical sector formed by observables. We show that observables of the vector model have an expected behavior on both classical and quantum level. Comparing quantum mechanics of the vector model with that of Dirac equation in Section 15.4, we obtain the rules for computation of probabilities and mean values of the vector model observables using the Dirac equation. The time evolution implied by the rules (414) turns out to be different from the ingenuous prescription (149). Hence the vector model of spin supports the point of view that the operators of the Dirac equation do not represent directly measurable quantities. 


\section{Spin-Tensor of Frenkel}

To construct the relativistic spinning particle, we need a Lorentz-covariant description of the spin fiber bundle (239). We note that our construction involves basic and target spaces as well as the map $f: \mathbb{R}^{6}(\boldsymbol{\omega}, \boldsymbol{\pi}) \rightarrow \mathbb{R}^{3}(\mathbf{S})$; see (108)-(110). We embed this $\mathrm{SO}(3)$-covariant construction into its suitably chosen $\mathrm{SO}(1,3)$-covariant extension. Let us start from the three-vector $\boldsymbol{\omega}$. We assume that relativistic spin can be described by a vector $\omega^{\mu}$ of Minkowski space such that $\omega^{\mu}=$ $(0, \boldsymbol{\omega})$ for the particle at rest in the laboratory frame. This condition expresses the Correspondence Principle: relativistic physics should approximate to the Newton physics in the limit of small velocities. To represent this condition in a covariant form in an arbitrary frame, we assume that in our model there exists a four-vector $p_{\mu}$ which for the particle at rest has the components $\left(p_{0}, \mathbf{0}\right)$. For the case of a free particle, the natural candidate is a vector proportional to the particle's four-velocity. For the particle in external field, the form of this vector is dictated by the structure of interaction; see below. With this $p^{\mu}$, the Lorentz-invariant statement $p \omega=0$ is equivalent to the condition that $\omega^{\mu}=(0, \boldsymbol{\omega})$ for the particle at rest. Following the same lines, we also assume the condition $p \pi=0$ for the conjugated momentum $\pi^{\mu}$ for $\omega^{\mu}$. Hence we replace the basic space $\mathbb{R}^{6}(\boldsymbol{\omega}, \boldsymbol{\pi})$ by direct product of two Minkowski spaces with the following natural action of the Lorentz group on it:

$$
\operatorname{SO}(1,3):\left(\begin{array}{l}
\omega \\
\pi
\end{array}\right) \longrightarrow\left(\begin{array}{l}
\omega^{\prime} \\
\pi^{\prime}
\end{array}\right)=\left(\begin{array}{ll}
\Lambda & 0 \\
0 & \Lambda
\end{array}\right)\left(\begin{array}{l}
\omega \\
\pi
\end{array}\right)
$$

The relativistic generalization of the surface (110) is given by the following $\mathrm{SO}(1,3)$-invariant surface of the phase space $\mathbb{M} \times \mathbb{M}$ :

$$
\mathbb{T}^{4}=\left\{\omega \pi=0, \pi^{2}-\frac{\alpha}{\omega^{2}}=0, \quad p \omega=0, \quad p \pi=0\right\} .
$$

Below we denote these constraints $T_{2}, T_{5}, T_{3}$ and $T_{4}$. As in nonrelativistic case, we have two first-class constraints $\omega \pi=0$ and $\pi^{2}-\alpha / \omega^{2}=0$. The constraints $p \omega=0$ and $p \pi=0$ are of second class, so we expect $8-2 \times 2-2=2$ physical degrees of freedom in the spin-sector.

It should be noted that $\omega^{\mu}$ and $\pi^{\mu}$ turn out to be spacelike vectors. Indeed, in the frame where $p^{\mu}=\left(p^{0}, \mathbf{0}\right)$ the constraints $p \omega=p \pi=0$ imply $\omega^{0}=\pi^{0}=0$. This implies $\omega^{2} \geq 0$ and $\pi^{2} \geq 0$. Then from the constraint $\pi^{2}-\alpha / \omega^{2}=0$ we conclude $\omega^{2}>0$ and $\pi^{2}>0$.

Let us consider the target space. To generalize the map $S^{i}=\epsilon^{i j k} \omega^{j} \pi^{k}$ to the case of four-dimensional quantities, we rewrite it in an equivalent form, using the known isomorphism among three-vector and antisymmetric $3 \times 3$-matrices

$$
\begin{aligned}
S^{i} & =\frac{1}{4} \epsilon^{i j k} S^{j k}, \\
\text { then } S^{i j} & =2 \epsilon^{i j k} S^{k} .
\end{aligned}
$$

Then

$$
\begin{gathered}
\qquad S^{i}=\epsilon^{i j k} \omega^{j} \pi^{k}, \\
\text { is equivalent to } S^{i j}=2\left(\omega^{i} \pi^{j}-\omega^{j} \pi^{i}\right) .
\end{gathered}
$$

The last equality has an evident generalization to the fourdimensional case: $S^{\mu \nu}=2\left(\omega^{\mu} \pi^{\nu}-\omega^{\nu} \pi^{\mu}\right)$. Hence the target space $\mathbb{R}^{3}(\mathbf{S})$ should be extended to the six-dimensional space $\mathbb{R}^{6}(\mathbf{D}, \mathbf{S})$ of antisymmetric $4 \times 4$ matrices. We present them as follows:

$$
S^{\mu \nu}(\mathbf{D}, \mathbf{S})=\left(\begin{array}{cccc}
0 & -D_{1} & -D_{2} & -D_{3} \\
D_{1} & 0 & 2 S_{3} & -2 S_{2} \\
D_{2} & -2 S_{3} & 0 & 2 S_{1} \\
D_{3} & 2 S_{2} & -2 S_{1} & 0
\end{array}\right),
$$

or, equivalently,

$$
S^{\mu \nu}=\left(S^{i 0}=D^{i}, S^{i j}=2 \epsilon^{i j k} S^{k}\right) .
$$

Lorentz group naturally acts on this space

$$
\begin{aligned}
\mathrm{SO}(1,3): S^{\mu \nu}(\mathbf{D}, \mathbf{S}) & \longrightarrow \\
S^{\mu \nu}\left(\mathbf{D}^{\prime}, \mathbf{S}^{\prime}\right) & =\Lambda^{\mu}{ }_{\alpha} \Lambda^{\nu}{ }_{\beta} S^{\alpha \beta}(\mathbf{D}, \mathbf{S}) .
\end{aligned}
$$

This equation determines transformation rules of the columns $\mathbf{D}$ and $\mathbf{S}$. They transform as three-vectors under the subgroup of rotations of the Lorentz group. The embedding (155) of three-dimensional spin-vector $\mathbf{S}$ into the fourdimensional spin-tensor has been suggested by Frenkel [13]. So we call $S^{\mu \nu}$ the Frenkel spin-tensor. The vector $\mathbf{D}$ is called dipole electric moment of the particle [17].

Now we are ready to define the covariant version of the map (109)

$$
\begin{aligned}
f: \mathbb{M}\left(\omega^{\mu}\right) \times \mathbb{M}\left(\pi^{\nu}\right) & \longrightarrow \mathbb{R}^{6}\left(S^{\mu \nu}\right) ; \\
\left(\omega^{\mu}, \pi^{\nu}\right) & \longrightarrow S^{\mu \nu}=2\left(\omega^{\mu} \pi^{\nu}-\omega^{\nu} \pi^{\mu}\right) .
\end{aligned}
$$

It has rank which equals 5 and maps a point of $\mathbb{M} \times \mathbb{M}$ to a pair of orthogonal three-dimensional vectors, $\mathbf{D S}=0$. By construction, $f$ is compatible with the transformations (150) and $(156)$ of $S O(1,3)$ : if $S^{\prime \mu \nu}(\mathbf{D}, \mathbf{S})=2\left(\omega^{\prime \mu} \pi^{\prime \nu}-\omega^{\prime \nu} \pi^{\prime \mu}\right)$, then $S^{\mu \nu}(\mathbf{D}, \mathbf{S})=2\left(\omega^{\mu} \pi^{\nu}-\omega^{\nu} \pi^{\mu}\right)$.

If $\mathbb{M} \times \mathbb{M}$ is considered as a symplectic space with canonical Poisson bracket, $\left\{\omega^{\mu}, \pi^{\nu}\right\}=\eta^{\mu \nu}$, the map $f$ induces $\mathrm{SO}(1,3)$ Lie-Poisson bracket on $\mathbb{R}^{6}$ :

$$
\begin{aligned}
& \left\{S^{\mu \nu}(\omega, \pi), S^{\alpha \beta}(\omega, \pi)\right\} \\
& \quad=2\left(\eta^{\mu \alpha} S^{\nu \beta}-\eta^{\mu \beta} S^{\nu \alpha}-\eta^{\nu \alpha} S^{\mu \beta}+\eta^{\nu \beta} S^{\mu \alpha}\right) .
\end{aligned}
$$

Consider the image $S^{\mu v}(\omega, \pi)$ of a point of the surface (151). Using the identity $S^{\mu \nu} S_{\mu \nu}=8\left(\omega^{2} \pi^{2}-(\omega \pi)^{2}\right)$ together with (151), we obtain five covariant equations which determine the spin-surface $\mathbb{S}^{2}$ in an arbitrary Lorentz frame

$$
\begin{aligned}
& S^{\mu \nu} S_{\mu \nu}=8 \alpha=6 \hbar^{2}, \\
& S^{\mu \nu} p_{\nu}=0 .
\end{aligned}
$$




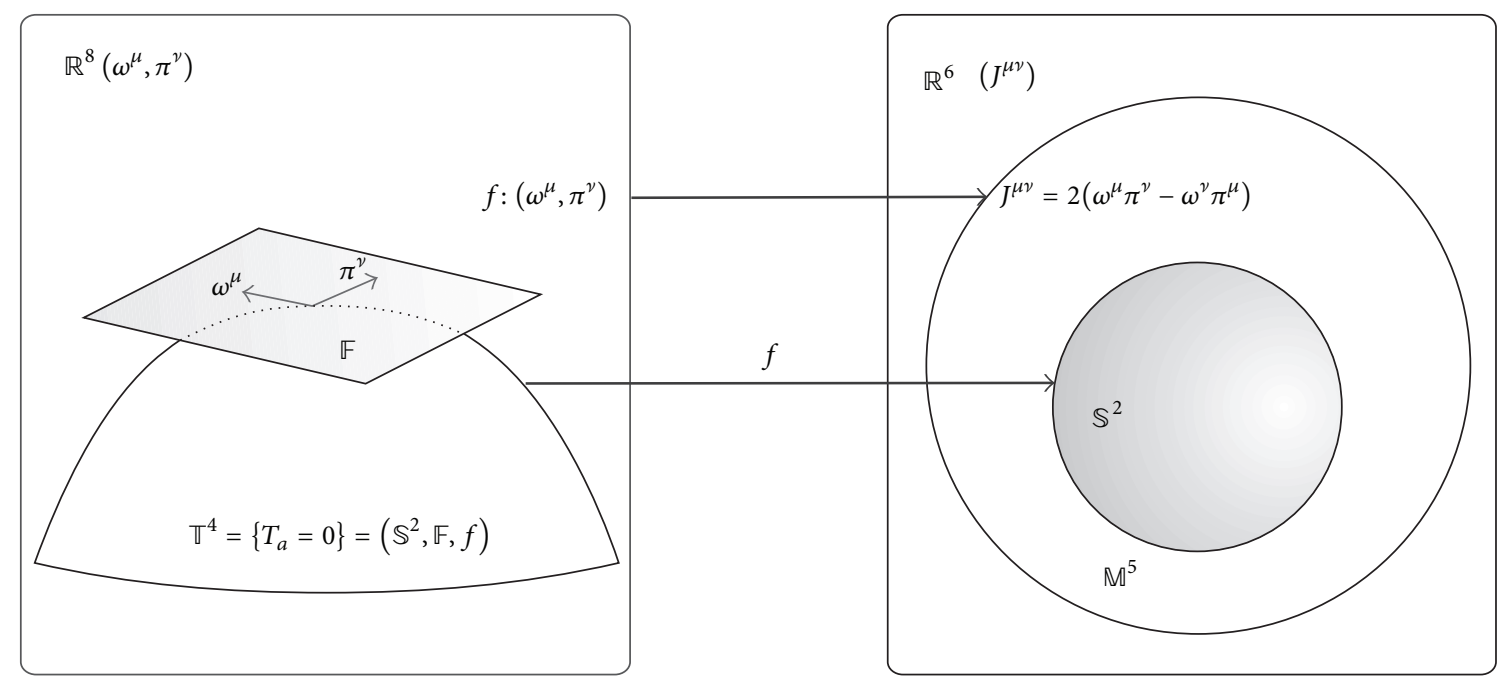

FIgURE 3: Fiber bundle $\mathbb{T}^{4}$ associated with relativistic spin.

As $\left(S^{\mu \nu} p_{\nu}\right) p_{\mu} \equiv 0$, we have only four independent equations imposed on six variables; therefore the spin-surface has dimension 2, as it should be. Denote $\mathbb{F}_{S} \in \mathbb{T}^{4}$ preimage of a point $S^{\mu \nu}$ of the base, $\mathbb{F}_{S}=f^{-1}\left(S^{\mu \nu}\right)$, that is, the standard fiber; see Figure 3. Its points are related by the structure group transformations (112) and (113).

Consider the rest frame of the vector $p^{\mu}$, that is, $p^{\mu}=$ $\left(p^{0}, \mathbf{0}\right)$, in this frame. The surface (151) acquires the form

$$
\begin{aligned}
\omega \pi & =0, \\
\pi^{2}-\frac{\alpha}{\omega^{2}} & =0, \\
\pi^{0} & =0, \\
\omega^{0} & =0
\end{aligned}
$$

and can be identified with the nonrelativistic spin-surface (239). Being restricted to this surface, the map (157) reads

$$
\begin{aligned}
\left.S^{\mu \nu}\right|_{\mathbb{T}^{4}} & =\left(\begin{array}{cccc}
0 & 0 & 0 & 0 \\
0 & 0 & 2 S_{3} & -2 S_{2} \\
0 & -2 S_{3} & 0 & 2 S_{1} \\
0 & 2 S_{2} & -2 S_{1} & 0
\end{array}\right), \\
\mathbf{S} & =\boldsymbol{\omega} \times \boldsymbol{\pi} .
\end{aligned}
$$

Hence in the rest frame the dipole electric moment vanishes, while the spatial part of spin-tensor coincides with the nonrelativistic spin. We conclude that $\mathrm{SO}(3)$-construction (108)-(239) is embedded into $\mathrm{SO}(1,3)$-covariant scheme. As in nonrelativistic case, the basic variables $\omega^{\mu}$ and $\pi_{v}$ do not represent observable quantities, only $S^{\mu \nu}$ does. This may be contrasted with $[34,81,82]$, where the equation $S^{\mu \nu}=$ 0 is assumed to be the first-class constraint of the Dirac formalism. In the result, $S^{\mu \nu}$ turns out to be unobservable quantity.

\section{Four-Dimensional Spin-Vector, Pauli- Lubanski Vector, and Bargmann-Michel- Telegdi Vector}

On the pure algebraic grounds, spin-tensor of Frenkel turns out to be equivalent to a four-dimensional vector. So the latter could also be used for the description of relativistic spin. Here we discuss the relevant formalism.

Levi-Civita symbol with $\epsilon^{0123}=1$ obeys the identities

$$
\begin{aligned}
& \epsilon^{a b c d} \epsilon_{a b \mu \nu}=-2\left(\delta_{\mu}^{c} \delta^{d}{ }_{\nu}-\delta^{c}{ }_{\nu} \delta^{d}{ }_{\mu}\right) \\
& \epsilon^{\mu a b c} \epsilon_{\mu i j k}=-\left[\delta^{a}{ }_{i}\left(\delta^{b}{ }_{j} \delta^{c}{ }_{k}-\delta^{b}{ }_{k} \delta^{c}{ }_{j}\right)\right. \\
& \left.\quad-\delta^{a}{ }_{j}\left(\delta^{b}{ }_{i} \delta^{c}{ }_{k}-\delta^{b}{ }_{k} \delta^{c}{ }_{i}\right)+\delta^{a}{ }_{k}\left(\delta^{b}{ }_{i} \delta^{c}{ }_{j}-\delta^{b}{ }_{j} \delta^{c}{ }_{i}\right)\right] .
\end{aligned}
$$

Given an antisymmetric matrix $J^{\mu \nu}=-J^{\nu \mu}$ and a vector $p^{\mu}$, we define the vectors

$$
s^{\mu}=\frac{1}{4 \sqrt{-p^{2}}} \epsilon^{\mu \nu \alpha \beta} p_{\nu} J_{\alpha \beta},
$$

then $s^{\mu} p_{\mu}=0$;

$$
\Phi^{\mu}=J^{\mu \nu} p_{\nu}
$$

then $\Phi^{\mu} p_{\mu}=0$.

When $p$ and $J$ represent generators of the Poincaré group, the vector (165) is called Pauli-Lubanski vector. It turns out to be useful for the classification of irreducible representations of the Poincaré group [83-85]. 
The tensor $J^{\mu \nu}$ and its dual, ${ }^{*} J^{\mu \nu} \equiv(1 / 2) \epsilon^{\mu \nu a b} J_{a b}$, can be decomposed on these vectors as follows:

$$
\begin{aligned}
J^{\mu \nu} & =\frac{\Phi^{\mu} p^{\nu}-\Phi^{\nu} p^{\mu}}{p^{2}}-\frac{2}{\sqrt{-p^{2}}} \epsilon^{\mu \nu a b} p_{a} s_{b} \\
\epsilon^{\mu \nu a b} J_{a b} & =4 \frac{p^{\mu} s^{\nu}-p^{\nu} s^{\mu}}{\sqrt{-p^{2}}}-\frac{2}{p^{2}} \epsilon^{\mu \nu a b} p_{a} \Phi_{b} .
\end{aligned}
$$

To prove (167), we contract (164) with $p_{a} p^{i} J^{j k}$. Eq. (168) follows from (167) contracted with $\epsilon_{a b \mu v}$. The definitions imply the identity relating the square of $s^{\mu}$ with a "square" of $J^{\mu \nu}$

$$
s^{\mu} s_{\mu}=\frac{1}{8} J^{\mu \nu} J_{\mu \nu}-\frac{1}{4 p^{2}}\left(J^{\mu \nu} p_{\nu}\right)^{2}
$$

Frenkel spin-tensor obeys $S^{\mu \nu} p_{v}=0$, that is, $\Phi^{\mu}=0$, and can be used to construct four-vector of spin (below we also call it Pauli-Lubanski vector)

$$
s^{\mu}(\tau) \equiv \frac{1}{4 \sqrt{-p^{2}}} \epsilon^{\mu \nu \alpha \beta} p_{\gamma} S_{\alpha \beta} .
$$

In the free theory $p^{\mu}$ is independent of $S^{\mu \nu}$, so this equation is linear on $S^{\mu \nu}$ and can be inverted. According to (167) we have

$$
S^{\mu \nu}=-\frac{2}{\sqrt{-p^{2}}} \epsilon^{\mu \nu \alpha \beta} p_{\alpha} s_{\beta} ;
$$

that is, the two quantities are mathematically equivalent, and we could work with $s^{\mu}$ instead of $S^{\mu \nu}$. Equation (169) implies proportionality of their magnitudes. In the interacting theory $p^{\mu}$ contains $S^{\mu \nu}$, so (170) becomes a nonlinear equation.

Let us compare spatial components of $s^{\mu}$ with the nonrelativistic spin-vector $\mathbf{S}$. In the rest system of $p^{\mu}, p^{\mu}=\left(p^{0}, \mathbf{0}\right)$, $\sqrt{-p^{2}}=\left|p^{0}\right|$, we have $s^{0}=0$ and

$$
s^{i}=\frac{p^{0}}{4\left|p^{0}\right|} \epsilon^{i j k} S_{j k}=\frac{p^{0}}{\left|p^{0}\right|} S^{i} ;
$$

that is, the two vectors coincide. This explains our normalization for $s^{\mu}$, (165). Under the Lorentz boost, $\mathbf{S}$ transforms as the spatial part of a tensor whereas $s^{\mu}$ transforms as a four-vector. So the two spins are different in all Lorentz frames except the rest frame. The relation between them in an arbitrary frame follows from (171):

$$
S^{i}=\frac{p^{0}}{\sqrt{-p^{2}}}\left(\delta_{i j}-\frac{p_{i} p_{j}}{\left(p^{0}\right)^{2}}\right) s^{j} .
$$

A four-dimensional vector $s_{b m t}^{\mu}$ with the property $u_{\mu} s_{b m t}^{\mu}=0$, where $u_{\mu}$ represents a four-velocity of the particle, has been successively used by Bargmann Michel and Telegdi to analyze the spin precession in uniform magnetic field; see [86] for details. In our vector model, even in the case of interaction, the condition $p s=0$ implies $u s=0$. So we expect that our equations of motion for $s^{\mu}$ should represent a generalization of the Bargmann-Michel-Telegdi equations to the case of an arbitrary electromagnetic field.

In summary, the relativistic spin can be described by the Frenkel spin-tensor (154) composed by the dipole electric moment $\mathbf{D}$ and the spin $\mathbf{S}$. In our vector model the Frenkel tensor is a composite quantity; see (157). In the rest frame of the vector $p^{\mu}$ we have $\mathbf{D}=0$, while $\mathbf{S}$ coincides with the vector of nonrelativistic spin. Intuitively, the Frenkel tensor shows how the nonrelativistic spin looks like in an arbitrary Lorentz frame.

\section{Lagrangian of Relativistic Spinning Particle}

9.1. Variational Problem for the Prescribed Dirac's Constraints. In the previous section we have discussed only the spin-sector of a spinning particle. To construct a complete theory, we add the position $x^{\mu}(\tau)$ and its conjugated momentum $p^{\mu}(\tau)$ taken in an arbitrary parametrization $\tau$. This implies that we deal with the reparametrization-invariant theory. So besides the spin-sector constraints (151) we expect also the massshell condition $T_{1}=p^{2}+(m c)^{2}=0$. Let us look for the Hamiltonian action which could produce these constraints. According to general theory [63-65, 67], it has the form $\int d \tau p \dot{x}+\pi \dot{\omega}-\left(H_{0}+\lambda_{i} T_{i}\right)$ where $T_{i}$ are the primary constraints of the theory. We expect $H_{0}=0$ due to the reparametrization invariance. As the suitable primary constraints, let us take $p^{2}+(m c)^{2}+\pi^{2}-a / \omega^{2}, T_{2}, T_{3}$, and $T_{4}$. Thus we consider the Hamiltonian variational problem

$$
\begin{aligned}
S_{H} & =\int d \tau p \dot{x}+\pi \dot{\omega}-\left[\frac{\lambda_{1}}{2}\left(p^{2}+(m c)^{2}+\pi^{2}-\frac{\alpha}{\omega^{2}}\right)\right. \\
& \left.+\lambda_{2}(\omega \pi)+\lambda_{3}(p \omega)+\lambda_{4}(p \pi)\right] .
\end{aligned}
$$

Due to the Poisson bracket $\left\{T_{2}, T_{5}\right\}=2 T_{5}$, in this formulation $T_{5}=0$ appears as the secondary constraint. To arrive at the Lagrangian action, we could follow the standard prescription. Excluding the conjugate momenta from $S_{H}$ according to their equations of motion, we obtain an action with the auxiliary variables $\lambda_{i}$. Excluding them, one after another, we obtain various equivalent forms of the Lagrangian action. To simplify these computations, we proceed as follows. First, we note that the constraints $\omega \pi=0$ and $p \omega=0$ always appear from the Lagrangian which involves the projector $N$; that is, we use $N \dot{x}$ and $N \dot{\omega}$ instead of $\dot{x}$ and $\dot{\omega}$. So we set $\lambda_{2}=\lambda_{3}=0$ in (174). Second, we present the remaining terms in (174) in the matrix form

$$
\begin{aligned}
S= & \int d \tau(p, \pi)\left(\begin{array}{c}
\dot{x} \\
\dot{\omega}
\end{array}\right)-\frac{\lambda_{1}}{2}(p, \pi)\left(\begin{array}{cc}
\eta & \lambda \eta \\
\lambda \eta & \eta
\end{array}\right)\left(\begin{array}{l}
p \\
\pi
\end{array}\right) \\
& -\frac{\lambda_{1}}{2}\left[(m c)^{2}-\frac{\alpha}{\omega^{2}}\right],
\end{aligned}
$$


where $\lambda=\lambda_{4} / \lambda_{1}$. The matrix which appeared in (175) is invertible; the inverse matrix is

$$
\frac{1}{1-\lambda^{2}}\left(\begin{array}{cc}
\eta & -\lambda \eta \\
-\lambda \eta & \eta
\end{array}\right)
$$

Eq. (175) is the Hamiltonian variational problem of the form $p \dot{q}-\left(\lambda_{1} / 2\right)\left(p A p+M^{2}\right)$; the latter follows from the Lagrangian $-M \sqrt{-\dot{q} A^{-1} \dot{q}}$. This allows us to exclude the variable $\lambda_{1}$. As it was combined above, we then replace $\dot{x}, \dot{\omega}$ by $N \dot{x}, N \dot{\omega}$ and obtain

$$
\begin{aligned}
S & =-\int d \tau \sqrt{(m c)^{2}-\frac{\alpha}{\omega^{2}}} \sqrt{-(N \dot{x}, N \dot{\omega})\left(\begin{array}{cc}
\frac{\eta}{1-\lambda^{2}} & \frac{-\lambda \eta}{1-\lambda^{2}} \\
\frac{-\lambda \eta}{1-\lambda^{2}} & \frac{\eta}{1-\lambda^{2}}
\end{array}\right)\left(\begin{array}{c}
N \dot{x} \\
N \dot{\omega}
\end{array}\right)} \\
& =-\int d \tau \sqrt{(m c)^{2}-\frac{\alpha}{\omega^{2}}} \sqrt{\left(1-\lambda^{2}\right)^{-1}[-\dot{x} N \dot{x}-\dot{\omega} N \dot{\omega}+2 \lambda \dot{x} N \dot{\omega}]} .
\end{aligned}
$$

To exclude the remaining auxiliary variable $\lambda$, we compute variation of (178) with respect to $\lambda$; this gives the equation

$$
(\dot{x} N \dot{\omega}) \lambda^{2}-(\dot{x} N \dot{x}+\dot{\omega} N \dot{\omega}) \lambda+(\dot{x} N \dot{\omega})=0,
$$

which determines $\lambda$

$$
=\frac{(\dot{x} N \dot{x}+\dot{\omega} N \dot{\omega}) \pm \sqrt{(\dot{x} N \dot{x}+\dot{\omega} N \dot{\omega})^{2}-4(\dot{x} N \dot{\omega})^{2}}}{2(\dot{x} N \dot{\omega})} .
$$

We substitute $\lambda_{+}$into (178) and use $\lambda_{+} \lambda_{-}=1$. Then (178) turns into the following action:

$$
\begin{aligned}
S= & -\frac{1}{\sqrt{2}} \int d \tau \sqrt{m^{2} c^{2}-\frac{\alpha}{\omega^{2}}} \\
& \times \sqrt{-\dot{x} N \dot{x}-\dot{\omega} N \dot{\omega}+\sqrt{[\dot{x} N \dot{x}+\dot{\omega} N \dot{\omega}]^{2}-4(\dot{x} N \dot{\omega})^{2}}} .
\end{aligned}
$$

The matrix $N_{\mu \nu}$ is the projector on the plane orthogonal to $\omega^{\nu}$ :

$$
\begin{aligned}
N_{\mu \nu} & =\eta_{\mu \nu}-\frac{\omega_{\mu} \omega_{\nu}}{\omega^{2}}, \\
\text { then } N_{\mu \alpha} N^{\alpha \nu} & =N_{\mu}{ }^{\nu} \\
N_{\mu \nu} \omega^{\nu} & =0 .
\end{aligned}
$$

In the spinless limit, $\alpha=0$ and $\omega^{\mu}=0$, the functional (181) reduces to the expected Lagrangian of spinless particle, $-m c \sqrt{-\dot{x}^{\mu} \dot{x}_{\mu}}$. It is well known that the latter can be written in equivalent form using the auxiliary variable $\lambda(\tau)$ as follows: $(1 / 2 \lambda) \dot{x}^{2}-(\lambda / 2) m^{2} c^{2}$. Similarly to this, (181) can be presented in the equivalent form

$$
\begin{aligned}
S & =\int d \tau \frac{1}{4 \lambda_{1}}[\dot{x} N \dot{x}+\dot{\omega} N \dot{\omega} \\
& \left.-\sqrt{[\dot{x} N \dot{x}+\dot{\omega} N \dot{\omega}]^{2}-4(\dot{x} N \dot{\omega})^{2}}\right]-\frac{\lambda_{1}}{2}\left[(m c)^{2}\right. \\
& \left.-\frac{\alpha}{\omega^{2}}\right]
\end{aligned}
$$

In summary, besides the "minimal" Lagrangian (181) we have obtained two of its equivalent formulations given by (178) and (183). The Lagrangians provide the appearance of equation $p \pi=0$ as the primary constraint. In turn, this seems crucial to introduce an interaction consistent with the constraints.

9.2. Interaction and the Problem of Covariant Formalism. In the formulation (181) without auxiliary variables, our model admits the minimal interaction with electromagnetic field and with gravity. As we have detailedly shown below, this does not spoil the number and algebraic structure of constraints presented in the free theory. Interaction with an electromagnetic potential is achieved by adding the standard term

$$
S_{\text {int }}=\frac{e}{c} \int d \tau A_{\mu} \dot{x}^{\mu}
$$

The minimal interaction with gravity is achieved $[25,33]$ by covariantization of (181). We replace $\eta_{\mu \nu} \rightarrow g_{\mu \nu}$ and usual derivative by the covariant one:

$$
\dot{\omega}^{\mu} \longrightarrow \nabla \omega^{\mu}=\dot{\omega}^{\mu}+\Gamma_{\alpha \beta}^{\mu} \dot{x}^{\alpha} \omega^{\beta} .
$$

Velocities $\dot{x}^{\mu}, \nabla \omega^{\mu}$ and projector $N_{\mu \nu}$ transform like contravariant vectors and covariant tensor, so the action is manifestly invariant under the general-coordinate transformations.

To introduce an interaction of spin with electromagnetic field, we use [24] the formulation (183) with the auxiliary variable $\lambda_{1}$. We add to the action (183) the term (184) and replace

$$
\dot{\omega}^{\mu} \longrightarrow D \omega^{\mu} \equiv \dot{\omega}^{\mu}-\lambda_{1} \frac{e \mu}{c} F^{\mu \nu} \omega_{v}
$$

We have denoted $\mu=g / 2$, where $g$ is gyromagnetic ratio; this agreement simplifies many of equations below. So we restore $g$ only in the final answers. $\lambda_{1}$ in this expression provides the homogeneous transformation law of $D \omega$ under the reparametrizations, $D_{\tau^{\prime}} \omega^{\prime}=\left(\partial \tau / \partial \tau^{\prime}\right) D_{\tau} \omega$.

The interaction of spin with gravity through the gravimagnetic moment will be achieved in the formulation (178); see below. 
Concerning the interaction of spin with electromagnetic field, let us briefly discuss an issue with nearly a century of history that is not completely clarified so far. While the complete relativistic Hamiltonian of the covariant formulation will be obtained below, its part, which is linear on spin, can be predicted from symmetry considerations. Indeed, the only Lorentz and $U(1)$-invariant term which involves $F$ and $S$ is $F_{\mu \nu} S^{\mu \nu}$. Using the covariant condition (160) we obtain

$$
H_{\text {relspin }} \sim-\frac{e}{4 m c} F_{\mu \nu} S^{\mu \nu}=\frac{e}{m c}\left[\frac{1}{m c} \mathbf{S}[\mathbf{p} \times \mathbf{E}]-\mathbf{B S}\right] .
$$

This can be compared with spin part of the Hamiltonian (91) with $g=2$

$$
H_{\text {spin }}=\frac{e}{m c}\left[\frac{1}{2 m c} \mathbf{S}[\mathbf{p} \times \mathbf{E}]-\mathbf{B S}\right]
$$

They differ by the famous and troublesome factor (in discussing this factor often refer to Thomas precession [15]; we will not touch this delicate and controversial issue [86-88] because of the covariant formalism automatically accounting for the Thomas precession [89]) of $1 / 2$. The same conclusion follows from comparison of equations of motion of the two formulations [10]. As we saw in Section 5.1, the expression (188) has very strong experimental support. The question why a covariant formalism does not lead directly to the correct result has been raised in 1926 [13] and remains under discussion to date $[10,36,90]$.

Following the work [31], in Section 11 we show that the vector model provides an answer to this question on a pure classical ground, without appeal to the Thomas precession, Dirac equation, or Foldy-Wouthuysen transformation. In a few words it can be described as follows. The relativistic vector model involves second-class constraints $\left(T_{3}\right.$ and $T_{4}$ of (151)), which we take into account by passing from the Poisson to Dirac bracket. So in the covariant formulation we arrive at the relativistic Hamiltonian (187) accompanied by noncanonical classical brackets. To construct the quantum mechanics, we could work with the relativistic Hamiltonian, but in this case we need to find quantum realization of the noncanonical brackets. Equivalently, we can find the variables with canonical brackets and quantize them in the standard way. The relativistic Hamiltonian (187), when written in the canonical variables, just gives (188).

9.3. Particle with the Fundamental Length Scale. Our basic model yields the fixed value of spin, as it should be for an elementary particle. Let us present the modification which leads to the theory with unfixed spin, and, similarly to Hanson-Regge approach [34], with a mass-spin trajectory constraint. Consider the following Lagrangian:

$$
\begin{aligned}
& L=-\frac{m c}{\sqrt{2}} \\
& \cdot \sqrt{-\dot{x} N \dot{x}-l^{2} \frac{\dot{\omega} N \dot{\omega}}{\omega^{2}}+\sqrt{\left[\dot{x} N \dot{x}+l^{2} \frac{\dot{\omega} N \dot{\omega}}{\omega^{2}}\right]^{2}-4 l^{2} \frac{(\dot{x} N \dot{\omega})^{2}}{\omega^{2}}}}
\end{aligned}
$$

where $l$ is a parameter with the dimension of length. The Dirac procedure yields the Hamiltonian

$$
\begin{aligned}
H= & \frac{\lambda_{1}}{2}\left(p^{2}+m^{2} c^{2}+\frac{\pi^{2} \omega^{2}}{l^{2}}\right)+\lambda_{2}(\omega \pi)+\lambda_{3}(p \omega) \\
& +\lambda_{4}(p \pi)
\end{aligned}
$$

which turns out to be combination of the first-class constraints $p^{2}+m^{2} c^{2}+\pi^{2} \omega^{2} / l^{2}=0, \omega \pi=0$ and the second-class constraints $p \omega=0, p \pi=0$. The Dirac procedure stops on the first stage; that is, there are no of secondary constraints. As compared with (181), the first-class constraint $\pi^{2}-\alpha / \omega^{2}=0$ does not appear in the present model. Due to this, square of spin is not fixed, $S^{2}=8\left(\omega^{2} \pi^{2}-\omega \pi\right) \approx 8 \omega^{2} \pi^{2}$. Using this equality, the mass-shell constraint acquires the form similar to the string theory

$$
p^{2}+m^{2} c^{2}+\frac{1}{8 l^{2}} S^{2}=0 .
$$

It has a clear meaning: the energy of the particle grows with its spin. The model has four physical degrees of freedom in the spin-sector. As the independent gauge-invariant degrees of freedom, we can take three components $S^{i j}$ of the spin-tensor together with any one product of conjugate coordinates, for instance, $\omega^{0} \pi^{0}$.

9.4. Classification of Vector Models. While we concentrate on the model specified by (151), it is instructive to discuss other sets of constraints that could be used for construction of a spinning particle. Equation (169) relating the Poincaré and Lorentz spins

$$
\begin{aligned}
s^{\mu} s_{\mu} & =\frac{1}{8} S^{\mu \nu} S_{\mu \nu}-\frac{1}{4 p^{2}}\left(S^{\mu \nu} p_{\nu}\right)^{2} \\
= & \omega^{2} \pi^{2}-(\omega \pi)^{2} \\
& -\frac{1}{p^{2}}\left[\omega^{2}(p \pi)^{2}+\pi^{2}(p \omega)^{2}-2(p \omega)(p \pi)(\omega \pi)\right]
\end{aligned}
$$

turns out to be useful in what follows.

(1) Our basic model (151) with two degrees of freedom implies $S^{\mu \nu} p_{\nu}=0$. Then (192) implies proportionality of the two spins, $8 s^{2}=S^{2}$, whereas their magnitudes are fixed due to the constraints $T_{2}$ and $T_{5}$. The variables $x^{\mu}, p^{\mu}$ and $S^{\mu \nu}$ have vanishing brackets with first-class constraints, so they are candidates for observables.

(2) The model with the constraints $\omega^{2}=\alpha^{2}$ and $\pi^{2}=$ $\beta^{2}$ instead of $\pi^{2}=\alpha / \omega^{2}$ is essentially equivalent to the basic model. The relationship between two models can be probably established using the conversion scheme [91, 92].

(3) Let us replace $T_{3} \equiv p \omega=0$ by $T_{3}^{\prime} \equiv p \omega-$ $\sqrt{\omega^{2}}=0$ in the set (151). These constraints appear in the model of rigid particle. $T_{4}$ and $T_{5}$ can be taken as the secondclass constraints, while $T_{2}$ and $T_{3}^{\prime}$ form the first-class subset. As a consequence, the model has two degrees of freedom. The Poincaré and Lorentz spins are proportional and have fixed magnitudes. The variables $x^{\mu}$ and $S^{\mu \nu}$ have nonvanishing brackets with first-class constraints. After canonical 
quantization, the constraint $T_{3}^{\prime}=0$ turns into the Dirac equation. Hence this semiclassical model can be used to study the relation among classical observables and operators of the Dirac theory.

(4) Hanson and Regge developed their model of a relativistic top [34] on the base of antisymmetric tensor $S^{\mu \nu}$ without making any special assumptions on its inner structure. The tensor is subject to first-class constraints $S^{\mu \nu} p_{\nu}=0$. This implies phase space with $2 \times 6-2 \times 3=6$ degrees of freedom as well as proportional spins with unfixed magnitude. A similar vector model could be constructed starting from the Hamiltonian action

$$
\begin{aligned}
S_{H}= & \int d \tau p \dot{x}+\pi \dot{\omega}-\frac{\lambda_{1}}{2}\left[p^{2}+m^{2} c^{2}+f\left(S^{2}\right)\right] \\
& -\lambda_{\mu} S^{\mu \nu} p_{\nu},
\end{aligned}
$$

where $S^{\mu \nu}=2\left(\omega^{\mu} \pi^{\nu}-\omega^{\nu} \pi^{\mu}\right)$. The variables $x^{\mu}$ and $S^{\mu \nu}$ are not observable in this model.

(5) To avoid the unobservable character of original variables in the model (193), we could replace $S^{\mu \nu} p_{v}=0$ by the pair of second-class constraints $p \omega=p \pi=0$. They provide $S^{\mu \nu} p_{v}=0$ and $8-2=6$ degrees of freedom.

(6) Adding the first-class constraint $\omega \pi=0$ to the model of Item 5 we arrive at the Lagrangian (189) with four degrees of freedom.

(7) There are models based on the light-like vector $\omega^{\mu}[93$, 94]. Consider the first-class constraints

$$
\begin{aligned}
\omega^{2} & =0, \\
\omega \pi & =0, \\
\pi^{2}(p \omega)^{2} & =\text { const, } \\
\text { then } s^{2} & =\text { const, } \\
s^{2} & =0 .
\end{aligned}
$$

This implies two degrees of freedom. The Poincaré and Lorentz spins, while fixed, do not correlate with one another. The variables $x^{\mu}, S^{\mu \nu}$, and $s^{\mu}$ are not observable quantities. We note also that $S^{\mu \nu} p_{v} \neq 0$; this complicates the analysis of nonrelativistic limit.

(8) Let us replace $\pi^{2}(p \omega)^{2}=$ const by $\sqrt{\pi^{2}}(p \omega)=$ const in the set (194). Similarly to Item (3), this constraint may be classical analog of the Dirac equation. This model still has not been studied.

Common for the models (5-8) is the problem whether they admit an interaction with external fields. Concerning the Hanson-Regge model, in their work [34] they analyzed whether the spin-tensor interacts directly with an electromagnetic field and concluded on impossibility to construct the interaction in a closed form. In our vector model an electromagnetic field interacts with the part $\omega^{\mu}$ of the spin-tensor.

\section{Interaction with Electromagnetic Field}

In this rather technical section we demonstrate that our variational problem yields a model of spinning particle with expected properties. In particular, our equations of motion generalize approximate equations of Frenkel and BargmannMichel-Telegdi to the case of an arbitrary electromagnetic field.

10.1. Manifestly Covariant Hamiltonian Formulation. As we saw in previous section, interaction with an arbitrary electromagnetic field can be described within the action

$$
\begin{aligned}
S & =\int d \tau \frac{1}{4 \lambda}[\dot{x} N \dot{x}+D \omega N D \omega \\
& \left.-\sqrt{[\dot{x} N \dot{x}+D \omega N D \omega]^{2}-4(\dot{x} N D \omega)^{2}}\right] \\
& -\frac{\lambda}{2}\left(m^{2} c^{2}-\frac{\alpha}{\omega^{2}}\right)+\frac{e}{c} A \dot{x},
\end{aligned}
$$

where the term

$$
D \omega^{\mu} \equiv \dot{\omega}^{\mu}-\lambda \frac{e \mu}{c} F^{\mu \nu} \omega_{v}
$$

accounts the spin-field interaction.

Let us construct Hamiltonian formulation of the model. Conjugate momenta for $x^{\mu}, \omega^{\mu}$, and $\lambda$ are denoted as $p^{\mu}$, $\pi^{\mu}$ and $p_{\lambda}$. We use also the canonical momentum $\mathscr{P}^{\mu} \equiv$ $p^{\mu}-(e / c) A^{\mu}$. Contrary to $p^{\mu}$, the canonical momentum is $U(1)$ gauge-invariant quantity. Since $p_{\lambda}=\partial L / \partial \dot{\lambda}=0$, the momentum $p_{\lambda}$ represents the primary constraint, $p_{\lambda}=0$. Expressions for the remaining momenta, $p^{\mu}=\partial L / \partial \dot{x}_{\mu}$ and $\pi^{\mu}=\partial L / \partial \dot{\omega}_{\mu}$, can be written in the form

$$
\begin{aligned}
\mathscr{P}^{\mu} & =\frac{1}{2 \lambda}\left(N \dot{x}^{\mu}-K^{\mu}\right), \\
K^{\mu} & \equiv T^{-(1 / 2)}\left[(\dot{x} N \dot{x}+D \omega N D \omega)(N \dot{x})^{\mu}\right. \\
& \left.-2(\dot{x} N D \omega)(N D \omega)^{\mu}\right], \\
\pi^{\mu} & =\frac{1}{2 \lambda}\left(N D \omega^{\mu}-R^{\mu}\right), \\
R^{\mu} & \equiv T^{-(1 / 2)}\left[(\dot{x} N \dot{x}+D \omega N D \omega)(N D \omega)^{\mu}\right. \\
& \left.-2(\dot{x} N D \omega)(N \dot{x})^{\mu}\right],
\end{aligned}
$$

where $T=[\dot{x} N \dot{x}+D \omega N D \omega]^{2}-4(\dot{x} N D \omega)^{2}$. The functions $K^{\mu}$ and $R^{\mu}$ obey the following remarkable identities:

$$
\begin{aligned}
K^{2} & =\dot{x} N \dot{x}, \\
R^{2} & =D \omega N D \omega, \\
K R & =-\dot{x} N D \omega, \\
\dot{x} R+D \omega K & =0, \\
\dot{x} K+D \omega R & =T^{1 / 2} .
\end{aligned}
$$

Due to (182), contractions of the momenta with $\omega^{\mu}$ vanish; that is, we have the primary constraints $\omega \pi=0$ and $\mathscr{P} \omega=0$. One more primary constraint, $\mathscr{P}_{\pi}=0$, is implied by (198). 
Hence we deal with a theory with four primary constraints. Hamiltonian is obtained excluding velocities from the expression

$$
H=p \dot{x}+\pi \dot{\omega}-L+\lambda_{i} T_{i}
$$

where $\lambda_{i}$ are the Lagrangian multipliers for the primary constraints $T_{i}$. To obtain its manifest form, we note the equalities $\mathscr{P}^{2}=\left(1 / 2 \lambda^{2}\right)[\dot{x} N \dot{x}-\dot{x} K], \pi^{2}=\left(1 / 2 \lambda^{2}\right)[D \omega N D \omega-$ $D \omega R]$, and $\mathscr{P} \dot{x}+\pi D \omega=2 L_{1}$, where $L_{1}$ is the first line in (195). Then, using (198) we obtain

$$
\left(\mathscr{P}^{2}+\pi^{2}\right)=\frac{2}{\lambda} L_{1}
$$

Further, using (198) we have

$$
\begin{aligned}
p \dot{x}+\pi \dot{\omega} & \equiv \mathscr{P} \dot{x}+\frac{e}{c} A \dot{x}+\pi D \omega+\lambda \frac{e \mu}{c}(\pi F \omega) \\
& =2 L_{1}+\frac{e}{c} A \dot{x}-\lambda \frac{e \mu}{4 c}(F S)
\end{aligned}
$$

where the Frenkel spin-tensor $S^{\mu \nu}$ appeared. Using (201) and (200) in (199), the Hamiltonian reads

$$
\begin{aligned}
H= & \frac{\lambda}{2}\left(\mathscr{P}^{2}-\frac{e \mu}{2 c}(F S)+m^{2} c^{2}+\pi^{2}-\frac{\alpha}{\omega^{2}}\right) \\
& +\lambda_{2}(\omega \pi)+\lambda_{3}(\mathscr{P} \omega)+\lambda_{4}\left(\mathscr{P}_{\pi}\right)+\lambda_{0} p_{\lambda} .
\end{aligned}
$$

The fundamental Poisson brackets $\left\{x^{\mu}, p^{\nu}\right\}=\eta^{\mu \nu}$ and $\left\{\omega^{\mu}, \pi^{\nu}\right\}=\eta^{\mu \nu}$ imply

$$
\begin{aligned}
\left\{x^{\mu}, \mathscr{P}^{\nu}\right\} & =\eta^{\mu \nu}, \\
\left\{\mathscr{P}^{\mu}, \mathscr{P}^{\nu}\right\} & =\frac{e}{c} F^{\mu \nu}, \\
\left\{S^{\mu \nu}, S^{\alpha \beta}\right\} & =2\left(\eta^{\mu \alpha} S^{\nu \beta}-\eta^{\mu \beta} S^{\nu \alpha}-\eta^{\nu \alpha} S^{\mu \beta}+\eta^{\nu \beta} S^{\mu \alpha}\right), \\
\left\{S^{\alpha \beta}, \omega^{\mu}\right\} & =2 \eta^{\mu[\alpha} \omega^{\beta]}, \\
\left\{S^{\alpha \beta}, \pi^{\mu}\right\} & =2 \eta^{\mu[\alpha} \pi^{\beta]} .
\end{aligned}
$$

According to (204), the spin-tensor is generator of Lorentz algebra $\mathrm{SO}(1,3)$. As $\omega \pi, \omega^{2}$, and $\pi^{2}$ are Lorentz-invariants, they have vanishing Poisson brackets with $S^{\mu \nu}$. To reveal the higher-stage constraints we write $\dot{T}_{i}=\left\{T_{i}, H\right\}=0$. The Dirac procedure stops on third stage with the following equations:

$$
\begin{aligned}
& p_{\lambda}=0 \Longrightarrow \\
& T_{1} \equiv \mathscr{P}^{2}-\frac{e \mu}{2 c}(F S)+m^{2} c^{2}+\pi^{2}-\frac{\alpha}{\omega^{2}}=0 \Longrightarrow \\
& \lambda_{3} C+\lambda_{4} D=0 \\
& T_{2} \equiv(\omega \pi)=0 \Longrightarrow \\
& T_{5} \equiv \pi^{2}-\frac{\alpha}{\omega^{2}}=0, \\
& T_{3} \equiv(\mathscr{P} \omega)=0 \Longrightarrow \\
& \lambda_{4}=-\frac{2 \lambda c}{e} a C,
\end{aligned}
$$

$$
\begin{aligned}
& T_{4} \equiv(\mathscr{P} \pi)=0 \Longrightarrow \\
& \lambda_{3}=\frac{2 \lambda c}{e} a D .
\end{aligned}
$$

We have denoted

$$
\begin{aligned}
& C=-\frac{e(\mu-1)}{c}(\omega F \mathscr{P})+\frac{e \mu}{4 c}(\omega \partial)(F S), \\
& D=-\frac{e(\mu-1)}{c}(\pi F \mathscr{P})+\frac{e \mu}{4 c}(\pi \partial)(F S),
\end{aligned}
$$

and the function $a$ is written in (212). The last equation from (206) turns out to be a consequence of (208) and (209) and can be omitted. Due to the secondary constraint $T_{5}$ which appeared in (207) we can replace the constraint $T_{1}$ on the equivalent one

$$
T_{1} \equiv \mathscr{P}^{2}-\frac{e \mu}{2 c}(F S)+m^{2} c^{2}=0
$$

This can be compared with (142). The Dirac procedure revealed two secondary constraints written in (211) and (207) and fixed the Lagrangian multipliers $\lambda_{3}$ and $\lambda_{4}$; the latter can be substituted into the Hamiltonian. The multipliers $\lambda_{0}, \lambda_{2}$ and the auxiliary variable $\lambda$ have not been determined. $H$ vanishes on the complete constraint surface, as it should be in a reparametrization-invariant theory.

We summarized the algebra of Poisson brackets between constraints in Table 1 . The constraints $p_{\lambda}, T_{1}, T_{2}$, and $T_{5}$ form the first-class subset, while $T_{3}$ and $T_{4}$ represent a pair of second class. The presence of two primary first-class constraints $p_{\lambda}$ and $T_{2}$ is in correspondence with the fact that two Lagrangian multipliers remain undetermined within the Dirac procedure.

Below we will use the following notation. In the equation which relates velocity and canonical momentum will appear the matrix $T$ :

$$
\begin{aligned}
& T^{\mu \nu}=\eta^{\mu \nu}-(\mu-1) a(S F)^{\mu \nu} \\
& a=\frac{-2 e}{4 m^{2} c^{3}-e(2 \mu+1)(S F)} .
\end{aligned}
$$

Using the identity $S^{\mu \alpha} F_{\alpha \beta} S^{\beta \nu}=-(1 / 2)\left(S^{\alpha \beta} F_{\alpha \beta}\right) S^{\mu \nu}$ we find the inverse matrix

$$
\begin{aligned}
& \widetilde{T}^{\mu \nu}=\eta^{\mu \nu}+(\mu-1) b(S F)^{\mu \nu}, \\
& b=\frac{-2 e}{4 m^{2} c^{3}-3 e \mu(S F)} .
\end{aligned}
$$

The two functions are related as follows: $b=2 a[2+(\mu-$ 1) $a(S F)]^{-1}$. The vector $Z^{\mu}$ is defined by

$$
Z^{\mu}=\frac{b}{4 c} S^{\mu \sigma}\left(\partial_{\sigma} F_{\alpha \beta}\right) S^{\alpha \beta} \equiv \frac{b}{4 c} S^{\mu \sigma} \partial_{\sigma}(F S)
$$


TABLE 1: Algebra of constraints.

\begin{tabular}{lccccc}
\hline & $T_{1}$ & $T_{5}$ & $T_{2}$ & $T_{3}$ & $T_{4}$ \\
\hline$T_{1}=\mathscr{P}^{2}-\frac{\mu e}{2 c}(F S)+m^{2} c^{2}$ & 0 & 0 & 0 & $-2 C$ & $-2 D$ \\
$T_{5}=\pi^{2}-\frac{\alpha}{\omega^{2}}$ & 0 & 0 & $-2 T_{5} \approx 0$ & $-2 T_{4} \approx 0$ & $\frac{2 \alpha}{\left(\omega^{2}\right)^{2}} T_{3} \approx 0$ \\
$T_{2}=\omega \pi$ & 0 & $2 T_{5} \approx 0$ & 0 & $-T_{3} \approx 0$ & $T_{4} \approx 0$ \\
$T_{3}=\mathscr{P} \omega$ & $2 C$ & $2 T_{4} \approx 0$ & $T_{3} \approx 0$ & 0 & $T_{1}+\frac{e}{2 c a} \approx \frac{e}{2 c a}$ \\
$T_{4}=\mathscr{P} \pi$ & $2 D$ & $-\frac{2 \alpha}{\left(\omega^{2}\right)^{2}} T_{3} \approx 0$ & $-T_{4} \approx 0$ & $-T_{1}-\frac{e}{2 c a} \approx-\frac{e}{2 c a}$ & 0 \\
\hline
\end{tabular}

This vanishes for homogeneous field, $\partial F=0$. The evolution of the basic variables was obtained according the standard rule $\dot{z}=\{z, H\}$. The equations read

$$
\begin{aligned}
\dot{x}^{\mu} & =\lambda\left(T^{\mu}{ }_{\nu} \mathscr{P}^{\nu}+\frac{\mu c a}{b} Z^{\mu}\right), \\
\dot{\mathscr{P}}^{\mu} & =\frac{e}{c}(F \dot{x})^{\mu}+\lambda \frac{\mu e}{4 c} \partial^{\mu}(F S), \\
\dot{\omega}^{\mu} & =\lambda \frac{e \mu}{c}(F \omega)^{\mu}-\lambda \frac{2 c a C}{e} \mathscr{P}^{\mu}+\pi^{\mu}+\lambda_{2} \omega^{\mu}, \\
\dot{\pi}^{\mu} & =\lambda \frac{e \mu}{c}(F \pi)^{\mu}-\lambda \frac{2 c a D}{e} \mathscr{P}^{\mu}-\frac{\alpha}{\left(\omega^{2}\right)^{2}} \omega^{\mu}-\lambda_{5} 2 \pi^{\mu} .
\end{aligned}
$$

Neither constraints nor equations of motion determine the variables $\lambda$ and $\lambda_{2}$; that is, the interacting theory preserves both reparametrization and spin-plane symmetries of the free theory. As a consequence, all the basic variables have ambiguous evolution. $x^{\mu}$ and $\mathscr{P}^{\mu}$ have one-parametric ambiguity due to $\lambda$ while $\omega$ and $\pi$ have two-parametric ambiguity due to $\lambda$ and $\lambda_{2}$. The variables with ambiguous dynamics do not represent observable quantities, so we need to search for the variables that can be candidates for observables. We note that (216) imply an equation for $S^{\mu \nu}$ which does not contain $\lambda_{2}$ :

$$
\dot{S}^{\mu \nu}=\lambda \frac{e \mu}{c}(F S)^{[\mu \nu]}+2 \mathscr{P}^{[\mu} \dot{x}^{\nu]} .
$$

This proves that the spin-tensor is invariant under local spinplane symmetry. The remaining ambiguity due to $\lambda$ contained in (215) and (217) is related to reparametrization invariance and disappears when we work with physical dynamical variables $x^{i}(t)$. So we will work with $x^{\mu}, \mathscr{P}^{\mu}$, and $S^{\mu \nu}$. We note that our constraints imply the algebraic restrictions on spintensor

$$
\begin{aligned}
& S^{\mu \nu} \mathscr{P}_{\nu}=0, \\
& S^{\mu \nu} S_{\mu \nu}=8 \alpha .
\end{aligned}
$$

Equations (215) and (217), together with (218) form a closed system which determines evolution of a spinning particle.

The quantities $x^{\mu}, P^{\mu}$, and $S^{\mu \nu}$, being invariant under spin-plane symmetry, have vanishing brackets with the corresponding first-class constraints $T_{2}$ and $T_{5}$. So, obtaining equations for these quantities, we can omit the corresponding terms in the Hamiltonian (202). Further, we can construct the
Dirac bracket for the second-class pair $T_{3}$ and $T_{4}$. Since the Dirac bracket of a second-class constraint with any quantity vanishes, we can now omit $T_{3}$ and $T_{4}$ from (202). Then the relativistic Hamiltonian acquires an expected form (compare it with the square of Dirac equation (142))

$$
H=\frac{\lambda}{2}\left(\mathscr{P}^{2}-\frac{e \mu}{2 c}(F S)+m^{2} c^{2}\right) .
$$

Equations (215) and (217) follow from this $H$ with use of Dirac bracket, $\dot{z}=\{z, H\}_{D B}$. The Dirac brackets in physical time parametrization will be computed in Section 10.3. The brackets in arbitrary parametrization can be found in [30].

We could also use the constraint $S^{\mu \nu} \mathscr{P}_{\nu}=0$ to represent $S^{0 i}$ through $S^{i j}$; then

$$
H=\frac{\lambda}{2}\left(\mathscr{P}^{2}+\frac{e g}{c}\left[\frac{1}{\mathscr{P} 0} \mathbf{S}[\mathbf{p} \times \mathbf{E}]-\mathbf{B S}\right]+m^{2} c^{2}\right) .
$$

\subsection{Comparison with Approximate Equations of Frenkel and Bargmann-Michel-Telegdi}

10.2.1. Lagrangian Equations. We can exclude the momenta $\mathscr{P}$ and the auxiliary variable $\lambda$ from the equations of motion. This yields second-order equation for the particle's position. To achieve this, we solve the first equation from (215) with respect to $\mathscr{P}$ and use the identities $(S F Z)^{\mu}=-(1 / 2)(S F) Z^{\mu}$, $\widetilde{T}^{\mu}{ }_{\nu} Z^{\nu}=(b / a) Z^{\mu}$; this gives $\mathscr{P}^{\mu}=(1 / \lambda) \widetilde{T}^{\mu}{ }_{\nu} \dot{x}^{\nu}-\mu c Z^{\mu}$. Then the condition $S^{\mu \nu} \mathscr{P}_{\nu}=0$ reads $(1 / \lambda)(S \widetilde{T} \dot{x})^{\mu}=\mu c(S Z)^{\mu}$. Using this equality, $\mathscr{P}^{2}$ can be presented as $\mathscr{P}^{2}=\left(1 / \lambda^{2}\right)(\dot{x} G \dot{x})+$ $\mu^{2} c^{2} Z^{2}$, where the symmetric matrix appeared:

$$
\begin{aligned}
G_{\mu \nu} & =\left(\widetilde{T}^{T} \widetilde{T}\right)_{\mu \nu} \\
& =\left[\eta+b(\mu-1)(S F+F S)+b^{2}(\mu-1)^{2} F S S F\right]_{\mu \nu} .
\end{aligned}
$$

The matrix $G$ is composed of the Minkowski metric $\eta_{\mu \nu}$ plus spin and field-dependent contribution, $G_{\mu \nu}=\eta_{\mu \nu}+h_{\mu \nu}(S)$. So we call $G$ the effective metric induced along the world-line by interaction of spin with electromagnetic field. We substitute $\mathscr{P}^{2}$ into the constraint (211); this gives $\lambda$ :

$$
\begin{aligned}
\lambda & =\frac{\sqrt{-\dot{x} G \dot{x}}}{m_{r} c}, \\
m_{r}^{2} & =m^{2}-\frac{\mu e}{2 c^{3}}(F S)-\mu^{2} Z^{2} .
\end{aligned}
$$


This shows that the presence of $\lambda$ in (196) implies highly nonlinear interaction of spinning particle with electromagnetic field. The final expression of canonical momentum through velocity is

$$
\mathscr{P}^{\mu}=\frac{m_{r} c}{\sqrt{-\dot{x} G \dot{x}}}\left[\delta^{\mu}{ }_{\nu}+(\mu-1) b(S F)^{\mu}{ }_{\nu}\right] \dot{x}^{\nu}-\mu c Z^{\mu} .
$$

Using (222) and (223), we exclude $\mathscr{P}^{\mu}$ and $\lambda$ from the Hamiltonian equations (215), (217), and (218). This gives closed system of Lagrangian equations for the set $x, S$. It is convenient to work with reparametrization-invariant derivative

$$
D=\frac{1}{\sqrt{-\dot{x} G \dot{x}}} \frac{d}{d \tau} .
$$

Then we have the dynamical equations

$$
\begin{aligned}
D\left[m_{r}(\widetilde{T} D x)^{\mu}\right]= & \frac{e}{c^{2}}(F D x)^{\mu}+\frac{\mu e}{4 m_{r} c^{3}} \partial^{\mu}(S F) \\
& +\mu D Z^{\mu}, \\
D S^{\mu \nu}= & \frac{e \mu}{m_{r} c^{2}} F^{[\mu \alpha} S_{\alpha}^{\nu]} \\
& -2 b m_{r} c(\mu-1) D x^{[\mu}(S F D x)^{\nu]} \\
& +2 \mu c D x^{[\mu} Z^{\nu]},
\end{aligned}
$$

the Lagrangian counterpart of the condition $S^{\mu \nu} \mathscr{P}_{\nu}=0$,

$$
S^{\mu \nu}\left[\dot{x}^{\nu}+(\mu-1) b(S F \dot{x})_{v}-\frac{\mu \sqrt{-\dot{x} G \dot{x}}}{m_{r}} Z_{v}\right]=0,
$$

as well as the value of spin condition, $S^{\mu \nu} S_{\mu \nu}=8 \alpha$. The equations contains the effective (spin and positiondependent) mass $m_{r}$; this can lead to certain geometric effects [95].

In the absence of interaction we obtain an expected dynamics

$$
\begin{aligned}
\frac{d}{d \tau} \frac{\dot{x}^{\mu}}{\sqrt{-\dot{x}^{2}}} & =0, \\
\dot{S}^{\mu \nu} & =0, \\
S^{\mu \nu} \dot{x}_{v} & =0 .
\end{aligned}
$$

The trajectory is a straight line, while $S^{\mu \nu}$ is a constant tensor.

10.2.2. Discussion. Eq. (225) and (227) show how spin modifies the classical equation of a point particle subject to Lorentz force

$$
m \frac{d}{d \tau}\left(\frac{\dot{x}^{\mu}}{\sqrt{-\dot{x}^{2}}}\right)=\frac{e}{c^{2}}(F \dot{x})^{\mu} .
$$

Let us discuss qualitatively the corresponding contributions. Canonical momentum $\mathscr{P}^{\mu}=p^{\mu}-(e / c) A^{\mu}$ of a spinless particle is proportional to its velocity, $\mathscr{P}^{\mu}=\left(m c / \sqrt{-\dot{x}^{2}}\right) \dot{x}^{\mu}$.
Interaction of spin with electromagnetic field modifies the relation between the two quantities; see (223). Contribution of anomalous magnetic moment $\mu \neq 1$ to the difference between $\dot{x}^{\mu}$ and $\mathscr{P}^{\mu}$ is proportional to $J / c^{3} \sim \hbar / c^{3}$, while the term with a gradient of field is proportional to $J^{2} / c^{3} \sim \hbar^{2} / c^{3}$. The interaction also modifies the constraints. In particular, the condition $S^{\mu \nu} \dot{x}_{v}=0$ of a free theory turns into $S^{\mu \nu} \mathscr{P}_{\nu}=$ 0 with $\mathscr{P}_{v} \neq \dot{x}_{v}$. This has an important consequence. If we adopt the standard special relativity notions of time and distance, the components $S^{0 i}$ vanish in the frame $\mathscr{P}^{\mu}=$ $\left(\mathscr{P}^{0}, \overrightarrow{0}\right)$ instead of the rest frame. Hence our model predicts small dipole electric moment of the particle immersed in an external field (for experimental estimations, see [96]).

Another important point is the emergence of an effective metric (221) for the particle in flat space. As we saw above, the incorporation of the constraints (218) into a variational problem and the search for an interaction consistent with them turn out to be rather nontrivial tasks, and the action (195) is probably the only solution of the problem. So, the appearance of effective metric (221) in equations of motion seems to be unavoidable in a systematically constructed model of spinning particle. An important consequences will be discussed in Section 12.

Summing up, in general case the Lorentz force is modified due to the presence of (time-dependent) radiation mass $m_{r}$ (222), the tetrad field $\widetilde{T}$, and the effective metric $G$ and due to two extra terms on right hand side of (225).

Consider the "classical" value of magnetic moment $\mu=1$. Then $\widetilde{T}=\eta$ and $G=\eta$. The Lorentz force is modified due to the presence of time-dependent radiation mass $m_{r}$, and two extra terms on right hand side of (225).

10.2.3. Homogeneous Field. The structure of our equations simplifies significantly for the homogeneous field $\partial_{\alpha} F^{\mu \nu}=0$, then $Z^{\mu}=0$. Contraction of (227) with $F_{\mu \nu}$ yields $(S F)^{\cdot}=0$; that is, $S^{\mu \nu} F_{\mu \nu}$ turns out to be the conserved quantity. This implies $\dot{m}_{r}=\dot{a}=\dot{b}=0$. Hence the Lorentz force is modified due to the presence of time-independent radiation mass $m_{r}$, the tetrad field $\widetilde{T}$, and the effective metric $G$. Equations (225) and (227) read

$$
\begin{aligned}
& \frac{d}{d \tau} \frac{\dot{x}^{\mu}}{\sqrt{-\dot{x} G \dot{x}}}=\frac{e}{m_{r} c^{2}}(T F \dot{x})^{\mu}-(T \dot{\widetilde{T}} \dot{x})^{\mu}, \\
& \dot{S}^{\mu \nu}=\frac{e \mu \sqrt{-\dot{x} G \dot{x}}}{m_{r} c^{2}} F_{\alpha}^{[\mu} S^{\alpha \nu]} \\
& -\frac{2 b m_{r} c(\mu-1)}{\sqrt{-\dot{x} G \dot{x}}} \dot{x}^{[\mu}(S F \dot{x})^{\nu]} \text {. }
\end{aligned}
$$

They simplify more in the parametrization which implies

$$
G_{\mu \nu} \dot{x}^{\mu} \dot{x}^{\nu}=-c^{2}
$$

Since $G \dot{x} \dot{x}=\dot{x}^{2}+O\left(S^{2}\right)$, in the linear approximation on $S$ this is just the proper-time parametrization.

The equations become even more simple when $\mu=g / 2=$ 1. Let us specify the equation of precession of spin to this 
case, taking physical time as the parameter, $\tau=t$. Then (227) reduces to the Frenkel condition, $S^{\mu \nu} \dot{x}_{v}=0$, while (226) reads $\dot{S}^{\mu \nu}=\left(e \sqrt{-\dot{x}^{2}} / m_{r} c^{2}\right)(F S)^{[\mu \nu]}$. We decompose spintensor on electric dipole moment $\mathbf{D}$ and Frenkel spin-vector $\mathbf{S}$ according to (155), then $\mathbf{D}=-(2 / \boldsymbol{c}) \mathbf{S} \times \mathbf{v}$, while variation rate of $\mathbf{S}$ is given by

$$
\frac{d \mathbf{S}}{d t}=\frac{e \sqrt{c^{2}-\mathbf{v}^{2}}}{m_{r} c^{3}}\{c \mathbf{S} \times \mathbf{B}-[\mathbf{E} \times[\mathbf{v} \times \mathbf{S}]]\} .
$$

Interaction with magnetic field yields precession of $\mathbf{S}$ around the vector $\mathbf{B}$, while interaction with electric field leads to an extra variation rate of $\mathbf{S}$ in the plane of vectors $\mathbf{v}$ and $\mathbf{S}$.

10.2.4. Comparison with Frenkel Equations. Frenkel found equations of motion consistent with the condition $S^{\mu \nu} \dot{x}_{v}=$ 0 up to order $O^{3}(S, F, \partial F)$. Besides, he considered the case $\mu=1$. Taking these approximations in our equations in the proper-time parametrization $\sqrt{-\dot{x}^{2}}=c$, we arrive at those of Frenkel (our $S$ is $2 m c / e$ of Frenkel $S$ ):

$$
\begin{aligned}
& \frac{d}{d \tau}\left[\left(m-\frac{e}{4 m c^{3}}(S F)\right) \dot{x}^{\mu}+\frac{e}{8 m^{2} c^{3}} S^{\mu \alpha} \partial_{\alpha}(S F)\right] \\
& =\frac{e}{c}(F \dot{x})^{\mu}+\frac{e}{4 m c} \partial^{\mu}(S F), \\
& \dot{S}^{\mu \nu}=\frac{e}{m c}\left[F_{\alpha}^{[\mu} S^{\alpha \nu]}-\frac{1}{4 m c^{2}} \dot{x}^{[\mu} S^{\nu] \alpha} \partial_{\alpha}(S F)\right], \\
& S^{\mu \nu} \dot{x}_{v}=0 .
\end{aligned}
$$

10.2.5. Comparison with Bargmann-Michel-Telegdi Equations. BMT-equations are

$$
\begin{aligned}
\ddot{x}^{\mu} & =\frac{e}{m c}(F \dot{x})^{\mu}, \\
\dot{s}^{\mu} & =\frac{e \mu}{m c}(F s)^{\mu}-\frac{e}{m c^{3}}(\mu-1)(s F \dot{x}) \dot{x}^{\mu}, \\
s^{\mu} \dot{x}_{\mu} & =0 .
\end{aligned}
$$

Obtaining their equations in homogeneous field, Bargmann, Michel, and Telegdi supposed that the motion of a particle is independent from the motion of spin. Besides they looked for the equation linear on $s^{\mu}$ and $F^{\mu \nu}$. It is convenient to introduce BMT-tensor dual to $s^{\mu}$ :

$$
S_{\mathrm{BMT}}^{\mu \nu}=\frac{2}{c} \epsilon^{\mu \nu \alpha \beta} s_{\alpha} \dot{x}_{\beta}
$$

Due to (236) this obeys the equation

$$
\dot{S}_{\mathrm{BMT}}^{\mu \nu}=\frac{e}{m c} F_{\alpha}^{[\mu} S_{\mathrm{BMT}}^{\alpha \nu]}+\frac{\mu-1}{c^{2}} \dot{x}^{[\mu}\left(S_{\mathrm{BMT}} F \dot{x}\right)^{\nu]} .
$$

Taking the proper-time parametrization and neglecting nonlinear terms on $F$ and $S$ in (230) and (231), we obtain (235) and (238).
10.2.6. Exact Solution to Equations of Motion in a Constant Magnetic Field. Comparing (230) and (235) we conclude that spin-field interaction modifies the Lorentz force equation even for the homogeneous magnetic field. To estimate the influence, it is convenient to work with four-dimensional spin-vector (170) instead of spin-tensor. The constraint $S^{\mu \nu} \mathscr{P}_{\nu}=0$ implies $s^{\mu} \dot{x}_{\mu}=0$, so $s^{\mu}$ can be identified with BMT-vector of spin. As a consequence of (215) and (217), it obeys the equation

$$
\dot{s}^{\mu}=\lambda \frac{e \mu}{c}\left[(F s)^{\mu}+\frac{1}{\mathscr{P}^{2}}(s F \mathscr{P}) \mathscr{P}^{\mu}\right]-\frac{1}{\mathscr{P}^{2}}(\dot{\mathscr{P}}) \mathscr{P}^{\mu}
$$

For the homogeneous magnetic field (215) and (239) have been solved exactly [30]; a qualitative picture of motion for $\mu \neq 1$ can be described as follows. Besides oscillations of spin first calculated by Bargmann, Michel, and Telegdi, the particle with anomalous magnetic moment experiences an effect of magnetic Zitterbewegung of the trajectory. Usual circular motion in the plane orthogonal to $\mathbf{B}$ is perturbed by slow oscillations along $\mathbf{B}$ with the amplitude of order of Compton wavelength, $\left(\overrightarrow{\mathscr{P}} / \mathscr{P}^{0}\right) \lambda_{C}$. The Larmor frequency and the frequency of spin oscillations are also shifted by small corrections.

10.3. Parametrization of Physical Time and Physical Hamiltonian. Equations for physical variables $x^{i}(t), \mathscr{P}^{i}(t)$, and $S^{\mu \nu}(t)$ follow from the formula of derivative of parametric function, $d z / d t=c\left(\dot{z} / \dot{x}^{0}\right)$, after the substitution of (215) and (217) on the right hand side. Our task here is to find a conventional Hamiltonian for these equations. Consider the Hamiltonian action associated with the Hamiltonian (202), $\int d \tau p \dot{x}+\pi \dot{\omega}-\lambda_{i} T_{i}$. The variational problem provides both equations of motion and constraints of the vector model in arbitrary parametrization. Using the reparametrization invariance of the functional, we take physical time as the evolution parameter, $\tau=x^{0} / c=t$; then the functional reads

$$
\begin{aligned}
S_{H} & =\int d t c \widetilde{\mathscr{P}}_{0}-e A^{0}+p_{i} \dot{x}^{i}+\pi_{\mu} \dot{\omega}^{\mu} \\
& -\frac{\lambda}{2}\left(-\widetilde{\mathscr{P}}_{0}^{2}+\mathscr{P}_{i}^{2}-\frac{e \mu}{2 c}(F S)+m^{2} c^{2}+\pi^{2}-\frac{\alpha}{\omega^{2}}\right) \\
& -\lambda_{i} T_{i},
\end{aligned}
$$

where it is convenient to denote $\widetilde{\mathscr{P}}_{0}=p_{0}-(e / c) A_{0}$. We can treat the term associated with $\lambda$ as a kinematic (that is velocity-independent) constraint of the problem. According to the standard classical-mechanics prescription [66], we solve the constraint

$$
\widetilde{\mathscr{P}}_{0}=-\widetilde{\mathscr{P}}^{0}=-\sqrt{\mathscr{P}_{i}^{2}-\frac{e \mu}{2 c}(F S)+m^{2} c^{2}+\pi^{2}-\frac{\alpha}{\omega^{2}}}
$$


TABle 2: Auxiliary Poisson brackets.

\begin{tabular}{|c|c|c|c|}
\hline & $\left\{\mathscr{P}^{0}, *\right\}$ & $\left\{T_{3}, *\right\}$ & $\left\{T_{4}, *\right\}$ \\
\hline$x^{i}$ & $-\frac{\mathscr{P}^{i}}{\mathscr{P}^{0}}$ & $-\omega^{i}+\frac{\omega^{0} \mathscr{P}^{i}}{\mathscr{P}^{0}}$ & $-\pi^{i}+\frac{\pi^{0} \mathscr{P}^{i}}{\mathscr{P}^{i}}$ \\
\hline $\mathscr{P}^{i}$ & $-\frac{e}{\mathscr{P}^{0} c}\left[(F \overrightarrow{\mathscr{P}})^{i}+\frac{\mu}{4} \partial^{i}(S F)\right.$ & $\frac{e \omega^{0}}{\mathscr{P}^{0} c}\left[(F \overrightarrow{\mathscr{P}})^{i}+\frac{\mu}{4} \partial^{i}(S F)\right]-\frac{e}{c}(F \vec{\omega})^{i}$ & $\frac{e \pi^{0}}{\mathscr{P}^{0} c}\left[(F \overrightarrow{\mathscr{P}})^{i}+\frac{\mu}{4} \partial^{i}(S F)\right]-\frac{e}{c}(F \vec{\pi})^{i}$ \\
\hline $\mathscr{P}^{0}$ & 0 & $\frac{e}{\mathscr{P}^{0} c}\left[(\mu-1)(\overrightarrow{\mathscr{P}} F \vec{\omega})+\frac{\mu}{4} \omega^{i} \partial^{i}(S F)-\mu F^{0 i} \mathscr{P}\left[0 \omega^{i]}\right]\right.$ & $\frac{e}{\mathscr{P}^{0} c}\left[(\mu-1)(\overrightarrow{\mathscr{P}} F \vec{\pi})+\frac{\mu}{4} \pi^{i} \partial^{i}(S F)-\mu F^{0 i} \mathscr{P}\left[0 \pi^{i]}\right.\right.$ \\
\hline$\omega^{\mu}$ & $-\frac{e \mu}{\mathscr{P}^{0} c}(F \omega)^{\mu}$ & $\frac{\omega^{0} e \mu}{\mathscr{P}^{0} c_{0}}(F \omega)^{\mu}$ & $-\mathscr{P}^{\mu}+\frac{\pi^{0} e \mu}{\mathscr{P}^{0} c}(F \omega)^{\mu}$ \\
\hline$\pi^{\mu}$ & $-\frac{e \mu}{\mathscr{P}^{0} c}(F \pi)^{\mu}$ & $\mathscr{P}^{\mu}+\frac{\omega^{0} e \mu}{\mathscr{P}^{0} \mathcal{C}}(F \pi)^{\mu}$ & $\frac{\pi^{0} e \mu}{\mathscr{P}^{0} c}(F \pi)^{\mu}$ \\
\hline$J^{\mu \nu}$ & $-\frac{e \mu}{\mathscr{P} 0} c(F S)^{[\mu v]}$ & $\frac{\omega^{0} e \mu}{\mathscr{P} 0} c(F S)^{[\mu \nu]}-2 \mathscr{P}^{[\mu} \omega^{\nu]}$ & $\frac{\pi^{0} e \mu}{\mathscr{P}^{0} c}(F S)^{[\mu \nu]}-2 \mathscr{P}^{[\mu} \pi^{\nu]}$ \\
\hline
\end{tabular}

and substitute the result back into (240); this gives an equivalent form of the functional

$$
\begin{aligned}
S_{H} & =\int d t p_{i} \dot{x}^{i}+\pi_{\mu} \dot{\omega}^{\mu} \\
& -\left[c \sqrt{\mathscr{P}_{i}^{2}-\frac{e \mu}{2 c}(F S)+m^{2} c^{2}+\pi^{2}-\frac{\alpha}{\omega^{2}}}+e A^{0}\right. \\
& \left.+\lambda_{2} \omega_{\mu} \pi^{\mu}+\lambda_{3} \mathscr{P}_{\mu} \omega^{\mu}+\lambda_{4} \mathscr{P}_{\mu} \pi^{\mu}\right],
\end{aligned}
$$

where the substitution (241) is implied in the last two terms as well. The expression in square brackets is the Hamiltonian. The sign in front of the square root in (241) was chosen according to the right spinless limit, $H=c \sqrt{\mathscr{P}_{i}^{2}+m^{2} c^{2}}+e A^{0}$. The variational problem implies the first-class constraints $T_{2}=\omega \pi=0, T_{5}=\pi^{2}-\alpha / \omega^{2}=0$ and the second-class constraints

$$
\begin{aligned}
& T_{3}=-\mathscr{P}^{0} \omega^{0}+\mathscr{P}^{i} \omega^{i}=0, \\
& T_{4}=-\mathscr{P}^{0} \pi^{0}+\mathscr{P}^{i} \pi^{i}=0,
\end{aligned}
$$

where

$$
\mathscr{P}^{0} \equiv \sqrt{\mathscr{P}_{i}^{2}-\frac{e \mu}{2 c}(F S)+m^{2} c^{2}} .
$$

In all expressions below the symbol $\mathscr{P}^{0}$ represents the function (244).

To represent the Hamiltonian from (242) in a more familiar form, we take into account the second-class constraints by passing from Poisson to Dirac bracket

$$
\begin{aligned}
\{A, B\}_{D}= & \{A, B\}-\left\{A, T_{3}\right\}\left\{T_{4}, T_{3}\right\}^{-1}\left\{T_{4}, B\right\} \\
& -\left\{A, T_{4}\right\}\left\{T_{3}, T_{4}\right\}^{-1}\left\{T_{3}, B\right\} .
\end{aligned}
$$

To compute the Dirac brackets of our variables, we use auxiliary Poisson brackets shown in Table 2. We will use the notation (212) and

$$
\begin{aligned}
u^{0} & =T_{\mu}^{0} \mathscr{P}^{\mu}+\frac{\mu c a}{b} Z^{0}, \\
\Delta^{\mu \nu} & =-\frac{2 c a}{e u^{0}} \mathscr{P}^{(0} S^{\mu \nu)},
\end{aligned}
$$

$$
\begin{aligned}
\mathscr{P}^{(0} S^{\mu \nu)} & =\mathscr{P}^{0} J^{\mu \nu}+\mathscr{P}^{\mu} S^{\nu 0}+\mathscr{P}^{\nu} S^{0 \mu}, \\
K^{\mu \nu} & =-\frac{\mu c a}{2 e u^{0}} S^{0 \mu} \partial^{\nu}(S F), \\
L^{\mu \nu \alpha} & =-\frac{2 \mu a}{u^{0}}(F S)^{[\mu \nu]} S^{0 \alpha}, \\
g^{\mu \nu} & =\eta^{\mu \nu}-\frac{2 c a \mathscr{P}^{0}}{e u^{0}} \mathscr{P}^{\mu} \mathscr{P}^{\nu} .
\end{aligned}
$$

Using the table, we obtain $\left\{T_{3}, T_{4}\right\}=e u^{0} / 2 c a \mathscr{P}^{0}$. Then Dirac brackets among the physical variables $x^{i}(t), \mathscr{P}^{i}(t)$, and $S^{\mu \nu}(t)$ are

$$
\begin{aligned}
& \left\{x^{i}, x^{j}\right\}_{D}=\frac{1}{2} \Delta^{i j}, \\
& \left\{x^{i}, \mathscr{P}^{j}\right\}_{D}=\delta^{i j}-\frac{e}{2 c}\left[\Delta^{i k} F^{k j}-K^{i j}\right], \\
& \left\{\mathscr{P}^{i}, \mathscr{P}^{j}\right\}_{D}=\frac{e}{c} F^{i j}-\frac{e^{2}}{2 c^{2}}\left[F^{i k} \Delta^{k n} F^{n j}-F^{[i k} K^{k j]}\right], \\
& \left\{S^{\mu \nu}, S^{\alpha \beta}\right\}_{D}=2\left(g^{\mu \alpha} S^{\nu \beta}-g^{\mu \beta} S^{\nu \alpha}-g^{\nu \alpha} S^{\mu \beta}\right. \\
& \left.\left.\quad+g^{\nu \beta} S^{\mu \alpha}\right)+L^{\mu \nu[\alpha} \mathscr{P} \beta\right] \\
& \left\{S^{\mu \nu}, x^{j}\right\}_{D}=\mathscr{P}\left[\mu \Delta^{\nu] j}+\frac{1}{2} L^{\mu \nu j},\right. \\
& \left\{S^{\mu \nu}, \mathscr{P}^{j}\right\}_{D}=\frac{e}{c}\left[-\mathscr{P}^{\mu}\left(\Delta^{\nu k} F^{k j}-K^{\nu j}\right)-(\mu \longleftrightarrow \nu)\right. \\
& \left.\quad+\frac{1}{2} L^{\mu \nu k} F^{k j}\right] .
\end{aligned}
$$

To continue, let us restrict to the case of a stationary electromagnetic field; then constraints do not depend explicitly on time. Dirac bracket of any quantity with secondclass constraints vanishes, so they can be omitted from the Hamiltonian. So we omit the last two terms in (242). The firstclass constraints $T_{2}$ and $T_{5}$ can be omitted as well, as they do 
not contribute into equations of motion for physical variables. In the result we obtain the physical Hamiltonian

$$
H_{\mathrm{ph}}=c \sqrt{\overrightarrow{\mathscr{P}}^{2}-\frac{e \mu}{2 c} F_{\mu \nu} S^{\mu \nu}+m^{2} c^{2}}+e A^{0} .
$$

The equations of motion that we discussed at the beginning of this section follow from this Hamiltonian according the rule $d z / d t=\left\{z, H_{\mathrm{ph}}\right\}_{D}$.

Note that the Dirac brackets encode most of the spin-field interaction, on this reason we have arrived at a rather simple form of physical Hamiltonian. The inclusion of an interaction into the geometry of phase-space and the resulting noncommutative geometry is under intensive investigation in various models [55, 95, 97-115].

\section{Spin-Induced Noncommutativity of Position and Fine Structure of Hydrogen Spectrum}

Here we discuss how the vector model resolves [31] the problem of covariant formalism described in Section 9.2.

To quantize our relativistic theory we need to find quantum realization of highly nonlinear classical brackets (247)-(251). They remain noncanonical even in absence of interaction. For instance, (247) in a free theory reads $\left\{x^{i}, x^{j}\right\}=\left(1 / 2 m c p^{0}\right) S^{i j}$. We emphasize that nonrelativistic model has canonical brackets (135), so the deformation arises as a relativistic correction induced by spin of a particle. Technically, the deformation is due to the fact that the constraints $p \omega=p \pi=0$ of relativistic theory, used to construct the Dirac bracket, mix up space-time and inner space coordinates.

Quantum realization of the brackets in a free theory will be obtained in Section 14, while in an interacting theory its explicit form is unknown. Therefore we quantize the interacting theory perturbatively, considering $c^{-1}$ as a small parameter and expanding all quantities in a power series. Let us consider the approximation $O\left(c^{-2}\right)$; that is, we neglect $c^{-3}$ and higher order terms. For the Hamiltonian (252) we have $H_{\mathrm{ph}} \approx m c^{2}+\mathscr{P}^{2} / 2 m-\mathscr{P}^{4} / 8 m^{3} c^{2}-(e \mu / 4 m c)(F S)$. Since the last term is of order $c^{-1}$, resolving the constraint $S^{\mu \nu} \mathscr{P}_{\nu}=0$ with respect to $S^{i 0}$ we can approximate $\mathscr{P}^{0}=m c$; then $S^{i 0}=(1 / m c) S^{i j} \mathscr{P}^{j}$. Using this expression together with (152) we obtain, up to order $c^{-2}$,

$$
\begin{aligned}
H_{\mathrm{ph}} \approx & m c^{2}+\frac{\mathscr{P}^{2}}{2 m}-\frac{\mathscr{P}^{4}}{8 m^{3} c^{2}}+e A^{0} \\
& +\frac{e \mu}{m c}\left[\frac{1}{m c} \mathbf{S}[\mathscr{P} \times \mathbf{E}]-\mathbf{B S}\right] .
\end{aligned}
$$

Due to the second and fourth terms, we need to know the operators $\widehat{\mathscr{P}}^{i}$ and $\widehat{x}^{i}$ up to order $c^{-2}$, while $\widehat{S}^{i j}$ should be found up to order $c^{-1}$. With this approximation, the commutators $[\widehat{x}, \widehat{x}],[\widehat{x}, \widehat{\mathscr{P}}]$, and $[\widehat{\mathscr{P}}, \widehat{\mathscr{P}}]$ can be computed up to order $c^{-2}$, while the remaining commutators can be written only up to $c^{-1}$. Therefore, we expand the right hand sides of Dirac brackets (247)-(251) in this approximation

$$
\begin{aligned}
\left\{x^{i}, x^{j}\right\}= & \frac{1}{2 m^{2} c^{2}} S^{i j}+O\left(\frac{1}{c^{3}}\right), \\
\left\{x^{i}, \mathscr{P}^{j}\right\}= & \delta^{i j}+O\left(\frac{1}{c^{3}}\right), \\
\left\{x^{i}, S^{j k}\right\}= & 0+O\left(\frac{1}{c^{2}}\right), \\
\left\{\mathscr{P}^{i}, \mathscr{P}^{j}\right\}= & \frac{e}{c} F^{i j}+O\left(\frac{1}{c^{4}}\right), \\
\left\{\mathscr{P}^{i}, S^{j k}\right\}= & O\left(\frac{1}{c^{3}}\right), \\
\left\{S^{i j}, S^{k l}\right\}= & 2\left(\delta^{i k} S^{j l}-\delta^{i l} S^{j \mathrm{k}}-\delta^{j k} S^{i l}+\delta^{j l} S^{i k}\right) \\
& +O\left(\frac{1}{c^{2}}\right) .
\end{aligned}
$$

An operator realization of these brackets reads

$$
\begin{aligned}
& \widehat{P}_{i}=-i \hbar \frac{\partial}{\partial x^{i}}-\frac{e}{c} A_{i}(\mathbf{x}), \\
& \widehat{x}_{i}=x_{i}-\frac{\hbar}{4 m^{2} c^{2}} \epsilon_{i j k} \widehat{P}^{j} \sigma^{k}, \\
& \hat{S}^{i j}=\hbar \epsilon_{i j k} \sigma_{k}
\end{aligned}
$$

then

$$
\begin{aligned}
\widehat{S}^{i} & =\frac{1}{4} \epsilon_{i j k} S^{j k}=\frac{\hbar}{2} \sigma^{i}, \\
\widehat{S}^{i 0} & =\frac{\hbar}{m c} \epsilon_{i j k} \widehat{P}^{j} \sigma^{k} .
\end{aligned}
$$

By construction of a Dirac bracket, the operator $\widehat{S}^{i 0}$ automatically obeys the desired commutators up to order $c^{-1}$. So we do not worry about this operator in the computations above.

We substitute these operators into the classical Hamiltonian (253). Expanding $A^{0}(\widehat{\mathbf{x}})$ in a power series, we obtain an additional contribution of order $c^{-2}$ to the potential due to noncommutativity of the position operator

$$
\begin{aligned}
& e A^{0}\left(x_{i}-(2 m c)^{-2} \epsilon_{i j k} \widehat{P}^{j} \widehat{S}^{k}\right) \\
& \quad \approx e A^{0}(\mathbf{x})-\frac{e}{2 m^{2} c^{2}} \widehat{\mathbf{S}}[\widehat{\mathbf{P}} \times \widehat{\mathbf{E}}] .
\end{aligned}
$$

The contribution has the same structure as fifth term in the Hamiltonian (253). In the result, the quantum Hamiltonian up to order $c^{-2}$ reads (we note that $\mu=g / 2$ )

$$
\begin{aligned}
\widehat{H}_{\mathrm{ph}}= & m c^{2}+\frac{\widehat{\mathbf{P}}^{2}}{2 m}-\frac{\widehat{\mathbf{P}}^{4}}{8 m^{3} c^{2}}+e A^{0} \\
& +\frac{e(g-1)}{2 m^{2} c^{2}} \widehat{\mathbf{S}}[\widehat{\mathbf{P}} \times \mathbf{E}]-\frac{e g}{2 m c} \mathbf{B} \widehat{\mathbf{S}} .
\end{aligned}
$$


The first three terms correspond to an increase of relativistic mass. The last two terms coincide with those in (91). In the result, we have shown that noncommutativity of electron's position in the vector model of spin is responsible for the fine structure of hydrogen atom.

We could carry out the same reasoning in classical theory, by asking on the new variables $z^{\prime}$ that obey the canonical brackets (135) as a consequence of (254). In the desired approximation they are $\mathscr{P}^{i}=\mathscr{P}^{\prime i}, x^{i}=x^{\prime i}-\left(1 / 4 m^{2} c^{2}\right) S^{\prime i j} \mathscr{P}^{\prime j}$, and $S^{i j}=S^{\prime i j}$; that is, the first relativistic corrections modify only the position variable.

\section{Ultrarelativistic Spinning Particle in Electromagnetic Background}

Let us compare the Lagrangian equations of spinning (225) and spinless (229) particle. For the spinning particle with $\mu \neq 1$, the relativistic-contraction factor (see (224)) contains the effective metric (221) instead of the Minkowski metric $\eta_{\mu \nu}$. In the result, equations for trajectory (225) and for precession of spin (226) became singular at the critical velocity which obeys the equation $\dot{x} G \dot{x}=0$. As we saw above, the singularity determines behavior of the particle in ultrarelativistic limit. The effective metric is composed of the Minkowski one plus (spin and field-dependent) contribution, $G=\eta+h(S)$. So we need to decide which of them should be used to construct the three-dimensional geometry discussed in Section 3. We first test the usual special relativity notions, $v^{i}=d x^{i} / d t$, $a^{i}=d v^{i} / d t$, and va $=v^{i} a^{i}$; that is, we suppose that the particle sees $\eta$ as the space-time metric. We show that in this case acceleration vanishes at the critical speed which is different from the speed of light. Then we estimate the ultrarelativistic limit using $G$ to define the three-dimensional geometry (20)-(23). Then $v_{\text {cr }}=c$, but since $G$ depends on spin, particles with different spins will probe slightly different three-dimensional geometries.

12.1. Ultrarelativistic Limit within the Usual Special Relativity Notions. It will be sufficient to estimate the acceleration in the uniform and stationary field. We take $\tau=t$ in (225)-(227) and compute the time derivative on l.h.s. of (225) with $\mu=$ $1,2,3$. Then the equations read

$$
\begin{aligned}
& a^{i}-\frac{v^{i}}{2(-v G v)} \frac{d}{d t}(-v G v) \\
& =T_{\nu}^{i}\left[\frac{e \sqrt{-v G v}}{m_{r} c^{2}}(F v)^{i}-\frac{d}{d t} \widetilde{T}_{\alpha}^{\nu} v^{\alpha}\right] \\
& \frac{d}{d t} S^{\mu v} \\
& =\frac{e \mu \sqrt{-v G v}}{m_{r} c^{2}}(F S)^{[\mu v]} \\
& \quad-\frac{2 b m_{r} c(\mu-1)}{\sqrt{-v G v} v^{[\mu}(S F v)^{\nu]}} \\
& (S v)^{\mu}+b(\mu-1)(S S F v)^{\mu}=0,
\end{aligned}
$$

where $v^{\mu}=(c, \mathbf{v})$. Eqs. (262) and (221) imply

$$
-v G v=-v \widetilde{T} v=c^{2}-\mathbf{v}^{2}-(\mu-1) b(v S F v) .
$$

We compute the time-derivatives in (260):

$$
\begin{aligned}
& \frac{d}{d t}(-v G v)=-2(\mathbf{v a})-(\mu-1) b\left\{[v(F S+S F)]_{i} a^{i}\right. \\
& +\frac{e \mu \sqrt{-v G v}}{m_{r} c^{2}}[(v F F S v)+(v F S F v)] \\
& \left.-\frac{2 b m_{r} c(\mu-1)}{\sqrt{-v g v}}\left[v^{2}(v F S F v)-(v S F v)(v F v)\right]\right\}, \\
& -T^{i}{ }_{\nu} \frac{d}{d t} \widetilde{T}_{\alpha}{ }_{\alpha} v^{\alpha}=-\frac{e \sqrt{-v G v}}{m_{r} c^{2}}\left\{\mu(\mu-1) b(F S F v)^{i}\right. \\
& \left.-\mu(\mu-1) a(S F F v)^{i}-\mu(\mu-1)^{2} a b(S F F S F v)^{i}\right\} \\
& +\frac{2 b m_{r} c(\mu-1)}{\sqrt{-v G v}} T^{i}{ }_{\nu}\left[v^{\nu}(v F S F v)-(S F v)^{v}(v F v)\right] .
\end{aligned}
$$

We note that all the potentially divergent terms (two last terms in (264) and in (265)), arising due to the contribution from $\dot{S} \sim(1 / \sqrt{-v G v})$, disappear on the symmetry grounds. We substitute nonvanishing terms into (260) obtaining the expression

$$
\begin{aligned}
& M_{j}^{i} a^{j}=\frac{e \sqrt{-v G v}}{m_{r} c^{2}}\left\{(F v)^{i}-\mu(\mu-1) b(F S F v)^{i}\right. \\
& +(\mu-1)^{2} a(S F F[\eta+\mu b S F] v)^{i} \\
& \left.\quad-v^{i} \frac{\mu(\mu-1) b}{2(-v G v)}(v F F S v)\right\},
\end{aligned}
$$

where the matrix

$$
\begin{aligned}
M_{j}^{i}=\delta_{j}^{i}+ & \frac{v^{i} v^{\mu} \Omega_{\mu j}}{2(-v G v)}, \\
& \text { with } \Omega_{\mu j}=2 \delta_{\mu j}+(\mu-1) b(F S+S F)_{\mu j}
\end{aligned}
$$

has the inverse

$$
\widetilde{M}_{j}^{i}=\delta_{j}^{i}-\frac{v^{i} v^{\mu} \Omega_{\mu j}}{2 c^{2}-(\mu-1) b v^{\mu}(F S+S F)_{\mu 0} v^{0}},
$$

with the property

$$
\widetilde{M}^{i}{ }_{j} v^{j}=v^{i} \frac{2(-v G v)}{2 c^{2}-(\mu-1) b v^{\mu}(F S+S F)_{\mu 0} v^{0}} .
$$

Applying the inverse matrix we obtain the acceleration

$$
\begin{aligned}
a^{i}= & \frac{e \sqrt{-v G v}}{m_{r} c^{2}}\left\{\widetilde { M } _ { j } ^ { i } \left[(F v)^{j}-\mu(\mu-1) b(F S F v)^{j}\right.\right. \\
& \left.+(\mu-1)^{2} a(S F F[\eta+\mu b S F] v)^{j}\right]-v^{i} \\
& \left.\cdot \frac{\mu(\mu-1) b(v F F S v)}{2 c^{2}-(\mu-1) b v^{\mu}(F S+S F)_{\mu 0} v^{0}}\right\} .
\end{aligned}
$$


For the particle with nonanomalous magnetic moment $(\mu=$ 1 ), the right hand side reduces to the Lorentz force, so the expression in braces is certainly nonvanishing in the ultrarelativistic limit. Thus the acceleration vanishes only when $v \rightarrow v_{\mathrm{cr}}$, where the critical velocity is determined by the equation $v G v=0$.

Let us estimate the critical velocity. Using the consequence $(\dot{x} S F \dot{x})=-b(\mu-1)(\dot{x} F S S F \dot{x})$ of the supplementary spin condition, and the expression $S^{\mu}{ }_{\alpha} S^{\alpha \nu}=-4\left[\pi^{2} \omega^{\mu} \omega^{\nu}+\right.$ $\left.\omega^{2} \pi^{\mu} \pi^{\nu}\right]$, we write

$$
\begin{aligned}
-(\dot{x} G \dot{x})= & c^{2}-\mathbf{v}^{2} \\
& +4 b^{2}(\mu-1)^{2}\left[\pi^{2}(\omega F \dot{x})^{2}+\omega^{2}(\pi F \dot{x})^{2}\right] .
\end{aligned}
$$

As $\pi$ and $\omega$ are space-like vectors, the last term is nonnegative, so $v_{\mathrm{cr}} \geq c$. We show that generally this term is nonvanishing function of velocity; then $v_{\mathrm{cr}}>c$. Assuming the contrary, that this term vanishes at some velocity, then

$$
\begin{aligned}
& \omega F \dot{x}=-\omega^{0}(\mathbf{E v})+(\boldsymbol{\omega}, c \mathbf{E}+\mathbf{v} \times \mathbf{B})=0 \\
& \pi F \dot{x}=-\pi^{0}(\mathbf{E v})+(\boldsymbol{\pi}, c \mathbf{E}+\mathbf{v} \times \mathbf{B})=0
\end{aligned}
$$

This implies $c(\mathbf{D E})+(\mathbf{D}, \mathbf{v} \times \mathbf{B})=0$. Consider the case $\mathbf{B}=$ 0 ; then it should be $(\mathbf{D E})=0$. On the other hand, for the homogeneous field the quantity $S^{\mu \nu} F_{\mu \nu}=2[(\mathrm{DE})+2(\mathrm{SB})]=$ $2(\mathrm{DE})$ is a constant of motion. Hence we can take the initial conditions for spin such that $(\mathrm{DE}) \neq 0$ at any instant; this implies $v_{\mathrm{cr}}>c$.

12.2. Ultrarelativistic Limit within the Geometry Determined by Effective Metric. As we saw above, if we insist on preserving the usual special relativity definitions of time and distance, the speed of light does not represent special point of the equation for trajectory. Acceleration of the particle with anomalous magnetic moment generally vanishes at the speed slightly higher than the speed of light. Hence we arrive at a rather surprising result that speed of light does not represent maximum velocity of the manifestly relativistic equation (266). This state of affairs is unsatisfactory because the Lorentz transformations have no sense above $c$, so two observers with relative velocity $c<v<v_{\text {cr }}$ will not be able to compare results of their measurements.

To keep the condition $v_{\mathrm{cr}}=c$, we use formal similarity of the matrix $G$ which appeared in (221) with space-time metric. Then we can follow the general relativity prescription of Section 3 to define time and distance in the presence of electromagnetic field. That is, we use $G$ of (221) to define the three-dimensional geometry (20)-(23). The effective metric depends on $x^{i}$ via the field strength $F\left(x^{0}, x^{i}\right)$, and on $x^{0}$ via the field strength as well as via the spin-tensor $S\left(x^{0}\right)$. So the effective metric is time-dependent even in stationary electromagnetic field. With these definitions we have, by construction, $-\dot{x} G \dot{x}=\left(d t / d x^{0}\right)^{2}\left(c^{2}-(\mathbf{v} \gamma \mathbf{v})\right)$, so the critical speed coincides with the speed of light. The intervals of time and distance are given now by (20) and (21); they slightly differ from those in empty space.
In the present case, the expression for three-acceleration can be obtained in closed form in an arbitrary electromagnetic field. We present (225) in the form (49)

$$
\begin{aligned}
& D D x^{\mu}=\mathscr{F}^{\mu}=-D x^{\mu} \frac{D m_{r}(S)}{m_{r}}-T^{\mu}{ }_{\nu} D \widetilde{T}_{\alpha}^{\nu}(S) D x^{\alpha} \\
& +T^{\mu}{ }_{\nu}\left\{\frac{e}{m_{r} c^{2}}(F D x)^{\nu}+\frac{\mu e}{4 m_{r}^{2} c^{3}} \partial^{\nu}(S F)\right. \\
& \left.+\frac{\mu}{m_{r}} D Z^{\nu}\right\} .
\end{aligned}
$$

Then the acceleration is given by (51). The first two terms on right hand side of (273) give potentially divergent contributions arising from the piece $\dot{S} \sim\left(1 / \sqrt{c^{2}-\mathbf{v} \gamma \mathbf{v}}\right)$ of (226). In the previous section we have seen that the dangerous contribution contained in the second term disappears. To analyze the first term, we substitute $\mathscr{F}^{i}$ from (273) into (51). With use the property $\widetilde{M}^{i}{ }_{j} v^{j}=v^{i}\left(\left(c^{2}-\mathbf{v} \gamma \mathbf{v}\right) / c^{2}\right)$, we obtain the acceleration

$$
\begin{aligned}
a^{i} & =\left(c^{2}-\mathbf{v} \gamma \mathbf{v}\right)\left[-v^{i} \frac{\dot{m}_{r}}{m_{r} c^{2}}-\frac{\widetilde{M}_{j}^{i} T^{j}{ }_{\nu} \dot{\widetilde{T}}_{\alpha}{ }^{\nu} v^{\alpha}}{c^{2}-\mathbf{v} \gamma \mathbf{v}}\right. \\
& +\widetilde{M}_{j}^{i} T^{j}{ }_{\nu}\left\{\frac{e}{m_{r} c^{2} \sqrt{c^{2}-\mathbf{v} \gamma \mathbf{v}}}(F v)^{\nu}\right. \\
& \left.\left.+\frac{\mu e}{4 m_{r}^{2} c^{3}} \partial^{\nu}(S F)+\frac{\mu}{m_{r} \sqrt{c^{2}-\mathbf{v} \gamma \mathbf{v}}} \dot{Z}^{\nu}\right\}\right] \\
& +\widetilde{M}_{j}^{i} \widetilde{\Gamma}_{k l}^{j}(\gamma) v^{k} v^{l}+\frac{1}{2}\left(\frac{d t}{d x^{0}}\right)^{-1}\left[\left(\mathbf{v} \partial_{0} \gamma \gamma^{-1}\right)^{i}\right. \\
& \left.-\frac{v^{i}}{c^{2}}\left(\mathbf{v} \partial_{0} \gamma \mathbf{v}\right)\right],
\end{aligned}
$$

so the divergency due to $\dot{m}_{r} \sim\left(1 / \sqrt{c^{2}-\mathbf{v} \gamma \mathbf{v}}\right)$ is cancelled by the factor in front of this term. In the result, the acceleration is finite as $v \rightarrow c$. Besides, taking into account the property $(\mathbf{v} \gamma)_{i} \widetilde{M}_{j}^{i}=(\mathbf{v} \gamma)_{j}\left(c^{2}-\mathbf{v} \gamma \mathbf{v} / c^{2}\right)$, we conclude that the longitudinal acceleration (52)

$$
\begin{aligned}
\mathbf{v} \gamma \mathbf{a} & =\frac{\left(c^{2}-\mathbf{v} \gamma \mathbf{v}\right)^{2}}{c^{2}}(\mathbf{v} \gamma \mathscr{F}) \\
+ & \frac{c^{2}-\mathbf{v} \gamma \mathbf{v}}{c^{2}}\left[(\mathbf{v} \gamma)_{i} \widetilde{\Gamma}_{k l}^{i}(\gamma) v^{k} v^{l}\right. \\
+ & \left.\frac{1}{2}\left(\frac{d t}{d x^{0}}\right)^{-1}\left(\mathbf{v} \partial_{0} \gamma \mathbf{v}\right)\right]
\end{aligned}
$$

vanishes in this limit. 
In summary, to preserve the equality $v_{\mathrm{cr}}=c$, we are forced to assume that particle in electromagnetic field probes the three-dimensional geometry determined with respect to the effective metric instead of the Minkowski metric. Similarly to Section 4, this implies rather unusual picture of the Universe filled with spinning matter. Since $G$ depends on spin, each particle will probe its own three-dimensional geometry. In principle this could be an observable effect. With the effective metric (221), (20) implies that the time of life of muon in electromagnetic field and in empty space should be different.

\section{Interaction with Gravitational Field}

13.1. Lagrangian of Spinning Particle with Gravimagnetic Moment. As we saw in Section 9.2, minimal interaction with gravitational field can be achieved by direct covariantization of the action (181). Remarkably, this leads to MPTD equations; see Section 13.2 below. Since they become problematic in ultrarelativistic regime, we are forced to look for a nonminimal interaction that could suitably modify the equations in this regime. To understand, how they might look, we use the remarkable analogy existing between the gravitational and electromagnetic fields. Hamiltonian formulations of the two minimally interacting theories become very similar if we identify electromagnetic field strength with the Riemann tensor contracted with spin, $F_{\mu \nu} \sim R_{\mu \nu \alpha \beta} S^{\alpha \beta}$. In particular, Hamiltonian action for both theories is

$$
\begin{aligned}
S_{H}= & \int d \tau p_{\mu} \dot{x}^{\mu}+\pi_{\mu} \dot{\omega}^{\mu} \\
& -\left[\frac{\lambda}{2}\left(P^{2}+(m c)^{2}+\pi^{2}-\frac{\alpha}{\omega^{2}}\right)+\lambda_{a} T_{a}\right],
\end{aligned}
$$

where $P_{\mu}=p_{\mu}-(e / c) A_{\mu}$ for electromagnetic field and $P_{\mu}=$ $p_{\mu}-\Gamma_{\alpha \mu}^{\beta} \omega^{\alpha} \pi_{\beta}$ for gravitational field. According to (202), nonminimal interaction through gyromagnetic ratio $2 \mu$ implies the contribution $-(e \mu / 2 c) F_{\mu \nu} S^{\mu \nu}$ into the third term. So we expect that nonminimal interaction with gravity could be achieved replacing this term by $\left(\lambda_{1} / 32\right) \kappa R_{\alpha \beta \mu \nu} S^{\alpha \beta} S^{\mu \nu}$. By analogy with the magnetic moment, the coupling constant $\kappa$ is called gravimagnetic moment $[10,36]$. Thus we consider the variational problem [27]

$$
\begin{aligned}
S_{\kappa} & =\int d \tau p_{\mu} \dot{x}^{\mu}+\pi_{\mu} \dot{\omega}^{\mu}-\left[\frac { \lambda _ { 1 } } { 2 } \left(P^{2}\right.\right. \\
& \left.+\kappa R_{\alpha \beta \mu \nu} \omega^{\alpha} \pi^{\beta} \omega^{\mu} \pi^{\nu}+(m c)^{2}+\pi^{2}-\frac{\alpha}{\omega^{2}}\right) \\
& \left.+\lambda_{2}(\omega \pi)+\lambda_{3}(P \omega)+\lambda_{4}(P \pi)\right],
\end{aligned}
$$

on the space of independent variables $x^{\mu}, p_{\nu}, \omega^{\mu}, \pi_{\nu}$, and $\lambda_{a}$.

Let us look for the Lagrangian which in the phase space implies the variational problem (277). First, we note that the constraints $\pi \omega=P \omega=0$ always appear from the Lagrangian which depends on $N \dot{x}$ and $N \dot{\omega}$ instead of $\dot{x}$ and $\dot{\omega}$. So we set $\lambda_{2}=\lambda_{3}=0$ in (277). Second, we present the remaining terms in (277) in the form

$$
\begin{aligned}
S_{\kappa}= & \int d \tau p_{\mu} \dot{x}^{\mu}+\pi_{\mu} \dot{\omega}^{\mu}-\frac{\lambda_{1}}{2}(P, \pi)\left(\begin{array}{cc}
g & \lambda g \\
\lambda g & \sigma
\end{array}\right)\left(\begin{array}{l}
P \\
\pi
\end{array}\right) \\
& -\frac{\lambda_{1}}{2}\left[(m c)^{2}-\frac{\alpha}{\omega^{2}}\right],
\end{aligned}
$$

where we have introduced the symmetric matrix

$$
\sigma^{\mu \nu}=g^{\mu \nu}+\kappa R_{\alpha}^{\mu}{ }^{\nu} \omega^{\alpha} \omega^{\beta},
$$

then $\sigma^{\mu \nu} \omega_{v}=\omega^{\mu}$.

The matrix which appeared in (278) is invertible; the inverse matrix is

$$
\left(\begin{array}{cc}
K \sigma & -\lambda K \\
-\lambda K & K
\end{array}\right), \quad \text { where } K=\left(\sigma-\lambda^{2} g\right)^{-1} .
$$

When $\kappa=0$ we have $K^{\mu \nu}=\left(1-\lambda^{2}\right)^{-1} g^{\mu \nu}$, and (280) coincides with the matrix which appeared in the free Lagrangian (177). Third, we note that the Hamiltonian variational problem of the form $p \dot{q}-\left(\lambda_{1} / 2\right) p A p$ follows from the reparametrizationinvariant Lagrangian $\sqrt{\dot{q} A^{-1} \dot{q}}$. So, we tentatively replace the matrix which appeared in (177) by (280) and switch on the minimal interaction of spin with gravity, $\dot{\omega} \rightarrow \nabla \omega$. This gives the following Lagrangian formulation of spinning particle with gravimagnetic moment [28]:

$$
\begin{aligned}
L & =-\sqrt{(m c)^{2}-\frac{\alpha}{\omega^{2}}} \sqrt{-(N \dot{x}, N \nabla \omega)\left(\begin{array}{cc}
K \sigma & -\lambda K \\
-\lambda K & K
\end{array}\right)\left(\begin{array}{c}
N \dot{x} \\
N \nabla \omega
\end{array}\right)} \\
& =-\sqrt{(m c)^{2}-\frac{\alpha}{\omega^{2}}} \sqrt{-\dot{x} N K \sigma N \dot{x}-\nabla \omega N K N \nabla \omega+2 \lambda \dot{x} N K N \nabla \omega} .
\end{aligned}
$$

Let us show that it does give the desired Hamiltonian formulation (277). The matrices $\sigma, K$, and $N$ are symmetric and mutually commuting. Canonical momentum for $\lambda$ vanishes and hence represents the primary constraint, $p_{\lambda}=0$. Conjugate momenta for $x^{\mu}$ and $\omega^{\mu}$ are $p_{\mu}=\partial L / \partial \dot{x}^{\mu}$ and $\pi_{\mu}=\partial L / \partial \dot{\omega}^{\mu}$, respectively. Due to the presence of Christoffel symbols in $\nabla \omega^{\mu}$, the conjugated momentum $p_{\mu}$ does not transform as a vector, so it is convenient to introduce the canonical momentum

$$
P_{\mu} \equiv p_{\mu}-\Gamma_{\alpha \mu}^{\beta} \omega^{\alpha} \pi_{\beta}
$$

the latter transforms as a vector under general transformations of coordinates. Manifest form of the momenta is as follows:

$$
\begin{aligned}
P_{\mu}= & \frac{1}{L_{0}}\left[m^{2} c^{2}-\frac{\alpha}{\omega^{2}}\right]^{1 / 2} \\
\cdot & {\left[(\dot{x} N K \sigma N)_{\mu}-\lambda(\nabla \omega N K N)_{\mu}\right], } \\
\pi_{\mu}= & \frac{1}{\sqrt{2} L_{0}}\left[m^{2} c^{2}-\frac{\alpha}{\omega^{2}}\right]^{1 / 2} \\
\cdot & {\left[(\nabla \omega N K N)_{\mu}-\lambda(\dot{x} N K N)_{\mu}\right], }
\end{aligned}
$$


where $L_{0}$ is the second square root in (282). They immediately imply the primary constraints $\omega \pi=0$ and $P \omega=0$. From the expressions

$$
\begin{aligned}
& P^{2}=\frac{1}{L_{0}^{2}}\left[(m c)^{2}-\frac{\alpha}{\omega^{2}}\right][(\dot{x} N K \sigma K \sigma N \dot{x}) \\
& \left.+\lambda^{2}(\nabla \omega N K K N \nabla \omega)-2 \lambda(\dot{x} N K \sigma K N \nabla \omega)\right], \\
& \pi \sigma \pi=\frac{1}{L_{0}^{2}}\left[(m c)^{2}-\frac{\alpha}{\omega^{2}}\right]\left[\lambda^{2}(\dot{x} N K \sigma K N \dot{x})\right. \\
& \quad+(\nabla \omega N K \sigma K N \nabla \omega)-2 \lambda(\dot{x} N K \sigma K N \nabla \omega)], \\
& 2 \lambda P \pi=\frac{1}{L_{0}^{2}}\left[(m c)^{2}-\frac{\alpha}{\omega^{2}}\right]\left[-2 \lambda^{2}(\dot{x} N K \sigma K N \dot{x})\right. \\
& \quad-2 \lambda^{2}(\nabla \omega N K K N \nabla \omega)+2 \lambda(\dot{x} N K \sigma K N \nabla \omega) \\
& \left.+2 \lambda^{3}(\dot{x} N K K N \nabla \omega)\right]
\end{aligned}
$$

we verify that their sum does not depend on velocities and hence gives one more constraint

$$
P^{2}+\pi \sigma \pi+2 \lambda P \pi=-\left[(m c)^{2}-\frac{\alpha}{\omega^{2}}\right] .
$$

Then Hamiltonian is $H=p \dot{x}+\pi \dot{\omega}-L+\lambda_{i} T_{i} \equiv P \dot{x}+$ $\pi \nabla \omega-L+\lambda_{i} T_{i}$, where the first and second terms have been identically rewritten in the general-covariant form. From (284) we obtain $H_{0}=P \dot{x}+\pi \nabla \omega-L=0$, so the Hamiltonian is composed of primary constraints

$$
\begin{aligned}
H & =\frac{\lambda_{1}}{2}\left[P^{2}+\kappa R_{\alpha \mu \beta \nu} \omega^{\alpha} \pi^{\mu} \omega^{\beta} \pi^{\nu}+(m c)^{2}+\pi^{2}-\frac{\alpha}{\omega^{2}}\right. \\
& +2 \lambda(P \pi)]+\lambda_{2}(\omega \pi)+\lambda_{3}(P \omega) .
\end{aligned}
$$

After the change of variables $\lambda \rightarrow \lambda_{4}=(1 / 2) \lambda_{1} \lambda$, we arrive at the same Hamiltonian as that which appeared in (277).

13.1.1. Hamiltonian Equations of Motion. Variation of the Hamiltonian action (277) with respect to $\lambda_{a}$ gives the algebraic equations

$$
\begin{aligned}
P^{2}+\kappa R_{\alpha \beta \mu \nu} \omega^{\alpha} \pi^{\beta} \omega^{\mu} \pi^{\nu}+(m c)^{2}+\pi^{2}-\frac{\alpha}{\omega^{2}} & =0, \\
\omega \pi & =0, \\
P \omega & =0, \\
P \pi & =0,
\end{aligned}
$$

while variations with respect to the remaining variables yield dynamical equations which can be written in the covariant form as follows:

$$
\begin{aligned}
& \frac{\delta S_{\kappa}}{\delta p_{\mu}}=0 \Longleftrightarrow \dot{x}^{\mu}=\lambda_{1} P^{\mu}+\lambda_{3} \omega^{\mu}+\lambda_{4} \pi^{\mu}, \\
& \frac{\delta S_{\kappa}}{\delta x^{\mu}}=0 \Longleftrightarrow \\
& \nabla P_{\mu}=-R_{\mu \nu \alpha \beta} \dot{x}^{\nu} \omega^{\alpha} \pi^{\beta}-\frac{1}{2} \lambda_{1} \kappa \nabla_{\mu} R_{\sigma \nu \alpha \beta} \omega^{\sigma} \pi^{\nu} \omega^{\alpha} \pi^{\beta},
\end{aligned}
$$

$$
\begin{aligned}
\frac{\delta S_{\kappa}}{\delta \pi_{\mu}}= & 0 \Longleftrightarrow \\
\nabla \omega^{\mu}= & \lambda_{1} \pi^{\mu}-\lambda_{1} \kappa R^{\mu}{ }_{\alpha \beta \nu} \omega^{\alpha} \omega^{\beta} \pi^{\nu}+\lambda_{2} \omega^{\mu}+\lambda_{4} P^{\mu}, \\
\frac{\delta S_{\kappa}}{\delta \omega^{\mu}}= & 0 \Longleftrightarrow \\
\nabla \pi_{\mu}= & -\frac{\lambda_{1} \alpha}{\omega^{4}} \omega_{\mu}-\lambda_{1} \kappa R_{\mu \nu \alpha \beta} \pi^{\nu} \omega^{\alpha} \pi^{\beta}-\lambda_{2} \pi_{\mu} \\
& -\lambda_{3} P_{\mu} .
\end{aligned}
$$

Eq. (290) has been repeatedly used to obtain the final form of equations (291)-(293). Computing time derivative of the algebraic equations (289) and using (290)-(293) we obtain the consequences

$$
\begin{aligned}
\pi^{2} & -\frac{\alpha}{\omega^{2}}=0, \\
\lambda_{3} & =4 a \lambda_{1}\left[2(1-\kappa) R_{\alpha \beta \mu \nu} \omega^{\alpha} \pi^{\beta} \pi^{\mu} P^{\nu}\right. \\
& \left.+\kappa \pi^{\sigma}\left(\nabla_{\sigma} R_{\mu \nu \alpha \beta}\right) \omega^{\mu} \pi^{\nu} \omega^{\alpha} \pi^{\beta}\right], \\
\lambda_{4} & =4 a \lambda_{1}\left[2(1-\kappa) R_{\alpha \beta \mu \nu} \omega^{\alpha} \pi^{\beta} \omega^{\mu} P^{\nu}\right. \\
& \left.+\kappa \omega^{\sigma}\left(\nabla_{\sigma} R_{\mu \nu \alpha \beta}\right) \omega^{\mu} \pi^{\nu} \omega^{\alpha} \pi^{\beta}\right] .
\end{aligned}
$$

Here and below we use the following notation. The gravitational analogy of electromagnetic field strength is denoted

$$
\theta_{\mu \nu}=R_{\mu \nu \alpha \beta} S^{\alpha \beta}
$$

In the equation which relates velocity and momentum will appear the matrix

$$
\begin{aligned}
T_{\nu}^{\alpha} \equiv \delta_{\nu}^{\alpha}-(\kappa-1) a S^{\alpha \sigma} \theta_{\sigma \nu}, & \\
a & =\frac{2}{16 m^{2} c^{2}+(\kappa+1)(S \theta)} .
\end{aligned}
$$

Using the identity

$$
(S \theta S)^{\mu \nu}=-\frac{1}{2}(S \theta) S^{\mu \nu}, \quad \text { where } S \theta=S^{\alpha \beta} \theta_{\alpha \beta},
$$

we find inverse of the matrix $T$

$$
\widetilde{T}_{\nu}^{\alpha} \equiv \delta_{\nu}^{\alpha}+(\kappa-1) b S^{\alpha \sigma} \theta_{\sigma v}, \quad b=\frac{1}{8 m^{2} c^{2}+\kappa(S \theta)} .
$$

The vector $Z^{\mu}$ is defined by

$$
Z^{\mu}=\frac{b}{8 c} S^{\mu \sigma}\left(\nabla_{\sigma} R_{\alpha \beta \rho \delta}\right) S^{\alpha \beta} S^{\rho \delta} \equiv \frac{b}{8 c} S^{\mu \sigma} \nabla_{\sigma}(S \theta) .
$$

This vanishes in a space with homogeneous curvature, $\nabla R=$ 0 .

The time-derivatives of (288), (294), and (295) do not yield new algebraic equations. Due to (294) we can replace the constraint (288) on $P^{2}+\kappa R_{\alpha \beta \mu \nu} \omega^{\alpha} \pi^{\beta} \omega^{\mu} \pi^{\nu}+(m c)^{2}=0$. The obtained expressions for $\lambda_{3}$ and $\lambda_{4}$ can be used to exclude 
these variables from (290)-(293). The constraints $T_{1}, T_{2}$, and $T_{5}$ form the first-class subset, while $T_{3}$ and $T_{4}$ represent a pair of second class.

Neither constraints nor equations of motion determine the functions $\lambda_{1}$ and $\lambda_{2}$; that is, the nonminimal interaction preserves both reparametrization and spin-plane symmetries of the theory. The presence of $\lambda_{1}$ and $\lambda_{2}$ in (292) and (293) implies that evolution of the basic variables is ambiguous, so they are not observable. To find the candidates for observables, we note once again that (292) and (293) imply an equation for $S^{\mu \nu}$ which does not contain $\lambda_{2}$. So we rewrite (290) and (291) in terms of spin-tensor and add to them the equation for $S^{\mu \nu}$; this gives the system

$$
\begin{aligned}
\dot{x}^{\mu} & =\lambda_{1}\left[T^{\mu}{ }_{\nu} P^{\nu}+\kappa \frac{a c}{b} Z^{\mu}\right], \\
\nabla P_{\mu} & =-\frac{1}{4} \theta_{\mu \nu} \dot{x}^{\nu}-\frac{\lambda_{1} \kappa}{32} \nabla_{\mu}(S \theta), \\
\nabla S^{\mu \nu} & =-\frac{\kappa \lambda_{1}}{4}(\theta S)^{[\mu \nu]}+2 P^{[\mu} \dot{x}^{\nu]} .
\end{aligned}
$$

Besides, the constraints (288), (289), and (294) imply

$$
\begin{aligned}
P^{2}+\frac{\kappa}{16} \theta S+(m c)^{2} & =0, \\
S^{\mu \nu} P_{\nu} & =0, \\
S^{\mu \nu} S_{\mu \nu} & =8 \alpha .
\end{aligned}
$$

Equations (305) imply that only two components of spintensor are independent, as it should be for spin one-half particle. Eq. (303), contrary to the equations for $\omega$ and $\pi$, does not depend on $\lambda_{2}$. This proves that the spin-tensor is invariant under local spin-plane symmetry. The remaining ambiguity due to $\lambda_{1}$ is related to reparametrization invariance and disappears when we work with physical dynamical variables $x^{i}(t)$. Equations (301)-(303), together with (304) and (305), form a closed system which determines evolution of a spinning particle with gravimagnetic moment.

The Hamiltonian equations can be equally obtained computing $\dot{z}=\{z, H\}$, where $z=(x, p, \omega, \pi)$, with the Hamiltonian given in square brackets of (277). Our original variables fulfill the usual Poisson brackets $\left\{x^{\mu}, p_{\nu}\right\}=\delta_{\nu}^{\mu}$ and $\left\{\omega^{\mu}, \pi_{\nu}\right\}=\delta_{\nu}^{\mu}$; then $\left\{P_{\mu}, P_{\nu}\right\}=R_{\lambda \mu \nu}^{\sigma} \pi_{\sigma} \omega^{\lambda},\left\{P_{\mu}, \omega^{\nu}\right\}=\Gamma_{\mu \alpha}^{\nu} \omega^{\alpha}$, $\left\{P_{\mu}, \pi_{\nu}\right\}=-\Gamma_{\mu \nu}^{\alpha} \pi_{\alpha}$. For the quantities $x^{\mu}, P^{\mu}$, and $S^{\mu \nu}$ these brackets imply

$$
\begin{aligned}
& \left\{x^{\mu}, P_{\nu}\right\}=\delta_{\nu}^{\mu}, \\
& \left\{P_{\mu}, P_{\nu}\right\}=-\frac{1}{4} R_{\mu \nu \alpha \beta} S^{\alpha \beta}, \\
& \left\{P_{\mu}, S^{\alpha \beta}\right\}=\Gamma_{\mu \sigma}^{\alpha} S^{\sigma \beta}-\Gamma_{\mu \sigma}^{\beta} S^{\sigma \alpha}, \\
& \left\{S^{\mu \nu}, S^{\alpha \beta}\right\} \\
& \quad=2\left(g^{\mu \alpha} S^{\nu \beta}-g^{\mu \beta} S^{\nu \alpha}-g^{\nu \alpha} S^{\mu \beta}+g^{\nu \beta} S^{\mu \alpha}\right) .
\end{aligned}
$$

We can simplify the Hamiltonian introducing the Dirac bracket constructed with help of second-class constraints

$$
\begin{aligned}
\{A, B\}_{D}= & \{A, B\} \\
& -\frac{1}{8 a}\left[\left\{A, T_{3}\right\}\left\{T_{4}, B\right\}-\left\{A, T_{4}\right\}\left\{T_{3}, B\right\}\right] .
\end{aligned}
$$

Since the Dirac bracket of a second-class constraint with any quantity vanishes, we can now omit $T_{3}$ and $T_{4}$ from the Hamiltonian. The quantities $x^{\mu}, P^{\mu}$, and $S^{\mu \nu}$, being invariant under spin-plane symmetry, have vanishing brackets with the first-class constraints $T_{2}$ and $T_{5}$. So, obtaining equations for these quantities, we can omit the last two terms in (277), arriving at the relativistic Hamiltonian

$$
H_{1}=\frac{\lambda_{1}}{2}\left(P^{2}+\frac{\kappa}{16}(\theta S)+m^{2} c^{2}\right) .
$$

Equations (301)-(303) can be obtained according the rule $\dot{z}=$ $\left\{z, H_{1}\right\}_{D}$.

We have obtained a rather simple expression for the Hamiltonian because most of the spin-gravity interaction is encoded in the Dirac brackets. The expression (308) together with the Dirac brackets could be an alternative starting point for computation of post-Newton corrections due to spin [81, 82].

13.1.2. Lagrangian Equations. Let us exclude $P^{\mu}$ and $\lambda_{1}$ from (302) and (303). Using (299) we solve (301) with respect to $P^{\mu}$. Using the resulting expression in the constraint (304) we obtain $\lambda_{1}$

$$
\begin{aligned}
\lambda_{1} & =\frac{\sqrt{-\dot{x} G \dot{x}}}{m_{r} c}, \\
\text { with } m_{r}^{2} & \equiv m^{2}+\frac{\kappa}{16 c^{2}}(S \theta)-\kappa^{2} Z^{2},
\end{aligned}
$$

where the effective metric now is given by

$$
G_{\mu \nu}=\widetilde{T}_{\mu}^{\alpha} g_{\alpha \beta} \widetilde{T}_{\nu}^{\beta}
$$

Then the expression for momentum in terms of velocity implied by (301) is

$$
P^{\mu}=\frac{m_{r} c}{\sqrt{-\dot{x} G \dot{x}}} \widetilde{T}_{\nu}^{\mu} \dot{x}^{\nu}-\kappa c Z^{\mu} .
$$

We substitute this $P^{\mu}$ into (302), (303):

$$
\begin{aligned}
\nabla\left[\frac{m_{r}}{\sqrt{-\dot{x} G \dot{x}}} \widetilde{T}^{\mu}{ }_{\nu} \dot{x}^{\nu}\right]= & -\frac{1}{4 c} \theta^{\mu}{ }_{\nu} \dot{x}^{\nu}-\kappa \frac{\sqrt{-\dot{x} G \dot{x}}}{32 m_{r} c^{2}} \nabla^{\mu}(S \theta) \\
& +\kappa \nabla Z^{\mu} \\
\nabla S^{\mu \nu}= & -\frac{\kappa \sqrt{-\dot{x} G \dot{x}}}{4 m_{r} c}(\theta S)^{[\mu \nu]} \\
& -\frac{2 m_{r} c(\kappa-1) b}{\sqrt{-\dot{x} G \dot{x}}} \dot{x}^{[\mu}(S \theta \dot{x})^{\nu]} \\
& +2 \kappa c \dot{x}^{[\mu} Z^{\nu]} .
\end{aligned}
$$


Together with (305), this gives us the Lagrangian equations for the spinning particle with gravimagnetic moment.

Comparing our equations to those of spinning particle on electromagnetic background (225)-(227), we see that the two systems have the same structure after the identification $\kappa \sim \mu$ and $\theta_{\mu \nu} \equiv R_{\mu \nu \alpha \beta} S^{\alpha \beta} \sim F_{\mu \nu}$, where $\mu$ is the magnetic moment. That is, a curvature influences trajectory of a spinning particle in the same way as an electromagnetic field with the strength $\theta_{\mu \nu}$.

13.2. MPTD Particle as the Spinning Particle without Gravimagnetic Moment. Let us compare MPTD equations (15)-(17) with equations of our spinning particle. Imposing $\kappa=0$ in (301)-(305), we write them in the form

$$
\begin{aligned}
P^{\mu} & =\frac{m c}{\sqrt{-\dot{x} G \dot{x}}}(\widetilde{T} \dot{x})^{\mu}, \\
\nabla P^{\mu} & =-\frac{1}{4}(\theta \dot{x})^{\mu}, \\
\nabla S^{\mu \nu} & =2 P^{[\mu} \dot{x}^{\nu]}, \\
S^{\mu \nu} P_{v} & =0 \\
P^{2}+(m c)^{2} & =0 \\
S^{2} & =8 \alpha,
\end{aligned}
$$

with $\widetilde{T}$ from (299) with $\kappa=0$. Comparing the systems, we see that our spinning particle has fixed values of square of spin and canonical momentum, while for MPTD particle these quantities represent constants of motion. We conclude that all the trajectories of a body with given $m$ and $S^{2}=\beta$ are described by our spinning particle with spin $\alpha=\beta / 8$ and with the mass equal to $\sqrt{m^{2}+\left(f^{2}(\beta) / c^{2}\right)}$. In this sense our spinning particle is equivalent to MPTD particle. We point out that our final conclusion remains true even we do not add (8) to MPTD equations: to study the class of trajectories of a body with $\sqrt{-P^{2}}=k$ and $S^{2}=\beta$ we take our spinning particle with $m=k / c$ and $\alpha=\beta / 8$. MPTD equations in the Lagrangian form (9)-(11) can be compared with (312)-(313).

Summing up, we demonstrated that MPTD equations correspond to the minimal interaction of spinning particle with gravity.

13.3. Consistency in Ultrarelativistic Regime Implies Quantized Gravimagnetic Moment. The paradoxical behavior of MPTD particle originates from the fact that variation rate of $\operatorname{spin}(60)$ diverges in the ultrarelativistic limit, $\nabla S \sim(1 / \sqrt{\dot{x} G \dot{x}})$ and contributes to the expression for acceleration (79) through the tetrad field $\widetilde{T}(S)$. Remarkably, for the nonminimal interaction with $\kappa=1$, the undesirable term in (313) vanishes. Besides this implies $\widetilde{T}_{\nu}^{\mu}=\delta^{\mu}{ }_{\nu}$ and $G_{\mu \nu}=g_{\mu \nu}$ and crucially simplifies the equations of motion (besides $S^{\mu \nu} P_{\nu}=0$, there are known others supplementary spin conditions $[5,6,8]$; in this respect we point out that the MPTD theory implies this condition with certain $P_{v}$ written in (15). Introducing $\kappa$, we effectively changed $P_{v}$ and hence changed the supplementary spin condition. For instance, when $\kappa=1$ and in the space with $\nabla R=0$, we have $P^{\mu}=(\widetilde{m} c / \sqrt{-\dot{x} g \dot{x}}) \dot{x}^{\mu}$ instead of (15).). The Hamiltonian equations (301)-(303) read

$$
\begin{aligned}
\frac{m_{r} c}{\sqrt{-\dot{x} g \dot{x}}} \dot{x}^{\mu} & =P^{\mu}+c Z^{\mu}, \\
\nabla P_{\mu} & =-\frac{1}{4} \theta_{\mu \nu} \dot{x}^{\nu}-\frac{\sqrt{-\dot{x} g \dot{x}}}{32 m_{r} c} \nabla_{\mu}(S \theta), \\
\nabla S^{\mu \nu} & =-\frac{\sqrt{-\dot{x} g \dot{x}}}{4 m_{r} c}(\theta S)^{[\mu \nu]}+2 P^{[\mu} \dot{x}^{\nu]} .
\end{aligned}
$$

Particle with unit gravimagnetic moment and MPTD particle have a qualitatively different behavior at low velocities. Indeed, keeping only the terms which may give a contribution in the leading post-Newton approximation, $\sim 1 / c^{2}$, we obtain from (316) and (317) the approximate equations

$$
\begin{aligned}
\nabla P_{\mu} & =-\frac{1}{4} \theta_{\mu \nu} \dot{x}^{\nu}-\frac{\sqrt{-\dot{x} g \dot{x}}}{32 m c}\left(\nabla_{\mu} R_{\alpha \beta \sigma \lambda}\right) S^{\alpha \beta} S^{\sigma \lambda}, \\
\nabla S^{\mu \nu} & =-\frac{\sqrt{-\dot{x} g \dot{x}}}{4 m c}(\theta S)^{[\mu \nu]},
\end{aligned}
$$

while MPTD equations in the same approximation read

$$
\begin{aligned}
\nabla P_{\mu} & =-\frac{1}{4} \theta_{\mu \nu} \dot{x}^{\nu}, \\
\nabla S^{\mu \nu} & =0 .
\end{aligned}
$$

Lagrangian equations are composed now by the equation for trajectory

$$
\begin{aligned}
\nabla\left[\frac{m_{r} \dot{x}^{\mu}}{\sqrt{-\dot{x} g \dot{x}}}\right]= & -\frac{1}{4 c} \theta^{\mu} \dot{x}^{\nu}-\frac{\sqrt{-\dot{x} g \dot{x}}}{32 m_{r} c^{2}} \nabla^{\mu}(S \theta) \\
& +\nabla Z^{\mu},
\end{aligned}
$$

and by the equation for precession of spin-tensor

$$
\nabla S^{\mu \nu}=-\frac{\sqrt{-\dot{x} g \dot{x}}}{4 m_{r} c}(\theta S)^{[\mu \nu]}+2 c \dot{x}^{[\mu} Z^{\nu]} .
$$

These equations can be compared with (59) and (60). In the modified theory:

(1) time interval and distance should be unambiguously defined within the original space-time metric $g_{\mu \nu}$. So the critical speed is equal to the speed of light;

(2) covariant precession of spin (321) has a smooth behavior, in particular, for covariantly constant curvature, $\nabla R=0$; we have $\nabla S \sim \sqrt{-\dot{x} g \dot{x}}$ contrary to $\nabla S \sim(1 / \sqrt{-\dot{x} g \dot{x}})$ in $(60)$;

(3) Spin ceases to affect the trajectory in ultrarelativistic limit: the trajectory of spinning particle becomes more and more close to that of spinless particle as $v \rightarrow c$. Besides, the spin precesses with finite angular velocity in this limit;

(4) Equation (320) in the space with covariantly constant curvature has the structure similar to (229); hence we expect that longitudinal acceleration vanishes as $v \rightarrow c$. Let us confirm this by direct computations. 
To find the acceleration, we separate derivative of the radiation mass $m_{r}$ and write equation (320) in the form

$$
\frac{d}{d \tau}\left[\frac{\dot{x}^{\mu}}{\sqrt{-\dot{x} g \dot{x}}}\right]=\frac{f^{\mu}}{\sqrt{-\dot{x} g \dot{x}}},
$$

where the force is

$$
\begin{aligned}
f^{\mu} \equiv & -\Gamma_{\alpha \beta}^{\mu} \dot{x}^{\alpha} \dot{x}^{\beta}-\frac{\sqrt{-\dot{x} g \dot{x}}}{4 m_{r} c} \theta^{\mu}{ }_{\nu} \dot{x}^{\nu}+\frac{\dot{x} g \dot{x}}{32 m_{r}^{2} c^{2}} \nabla^{\mu}(S \theta) \\
& +\frac{\sqrt{-\dot{x} g \dot{x}}}{m_{r}} \nabla Z^{\mu}-\dot{x}^{\mu} \frac{\dot{m}_{r}}{m_{r}} .
\end{aligned}
$$

While this expression contains derivatives of spin due to $\dot{m}_{r}$-term, the resulting expression is nonsingular function of velocity because $\nabla S$ is a smooth function. Hence, contrary to (70), the force now is nonsingular function of velocity. We take $\tau=x^{0}$ in the spatial part of the system (322); this gives

$$
\left(\frac{d t}{d x^{0}}\right)^{-1} \frac{d}{d x^{0}}\left[\frac{v^{i}}{\sqrt{c^{2}-(\mathbf{v} \gamma \mathbf{v})}}\right]=\frac{f^{i}(v)}{\sqrt{c^{2}-(\mathbf{v} \gamma \mathbf{v})}}
$$

where $f^{i}(v)$ is obtained from (323) replacing $\dot{x}^{\mu}$ by $v^{\mu}$ of (37). This system is of the form (36), so the acceleration is given by (43) and (44):

$$
\begin{aligned}
a^{i} & =\widetilde{M}_{j}^{i}\left[f^{j}+\widetilde{\Gamma}^{j}{ }_{k l}(\gamma) v^{k} v^{l}\right]+\frac{1}{2}\left(\frac{d t}{d x^{0}}\right)^{-1} \\
. & {\left[\left(\mathbf{v} \partial_{0} \gamma \gamma^{-1}\right)^{i}-\frac{\left(\mathbf{v} \partial_{0} \gamma \mathbf{v}\right)}{c^{2}} v^{i}\right], } \\
\mathbf{v} \gamma \mathbf{a} & =\left(1-\frac{\mathbf{v} \gamma \mathbf{v}}{c^{2}}\right)\left[(\mathbf{v} \gamma)_{i}\left[f^{i}(v)+\widetilde{\Gamma}_{k l}^{i}(\gamma) v^{k} v^{l}\right]\right. \\
& \left.+\frac{1}{2}\left(\frac{d t}{d x^{0}}\right)^{-1}\left(\mathbf{v} \partial_{0} \gamma \mathbf{v}\right)\right] .
\end{aligned}
$$

With the smooth $f^{i}$ given in (323), the acceleration (325) remains finite while the longitudinal acceleration (326) vanishes in the limit $v \rightarrow c$. Due to the identities (39), we have $(\mathbf{v} \gamma)_{i} f^{i} \stackrel{v \rightarrow c}{\longrightarrow}-(\mathbf{v} \gamma)_{i} \Gamma_{\alpha \beta}^{i} \dot{x}^{\alpha} \dot{x}^{\beta}$; that is, the trajectory tends to that of spinless particle in the limit.

In resume, contrary to MPTD equations, the modified theory is consistent with respect to the original metric $g_{\mu \nu}$. Hence the modified equations could be more promising for description of the rotating objects in astrophysics.

\section{One-Particle Relativistic Quantum Mechanics with Positive Energies, Canonical Formalism}

As we have seen above, on the classical level our vector model adequately describes spinning particle in arbitrary gravitational and electromagnetic fields. Moreover, taking into account the leading relativistic corrections in quantized theory with interaction, we have explained the famous one-half factor in nonrelativistic Hamiltonian (188); see Section 10.3. Now we turn to a systematic discussion of our model on the quantum level. In this section we construct quantum mechanics of the free theory (183) in the physical time parametrization. This yields the Schrödinger equation (354), with the Hamiltonian $c \sqrt{\mathbf{p}^{2}+(m c)^{2}}$ acting on a space of twocomponent wave functions. Note that all the solutions have positive energy. The novel point is that the naive expressions, $x^{i}$ and $\sigma^{i}$, do not represent operators of position and spin of our theory. This is due to the second-class constraints $p \omega=p \pi=0$ of the relativistic theory, which guarantee the supplementary spin condition $S^{\mu \nu} p_{v}=0$. The constraints should be taken into account with help of Dirac bracket; this implies a deformation of classical brackets which are subject to quantization. In the result, the position and spin of a spinning particle are represented by the operators (350) and (353). The remaining sections are devoted to establishing of Lorentz covariance of the obtained quantum mechanics.

In the free Lagrangian (183) it is convenient to rescale $\omega \rightarrow \sqrt{\lambda} \omega$; then

$$
\begin{aligned}
S= & \int d \tau \frac{1}{4 \lambda}[\dot{x} N \dot{x}+\lambda \dot{\omega} N \dot{\omega} \\
& \left.-\sqrt{[\dot{x} N \dot{x}+\lambda \dot{\omega} N \dot{\omega}]^{2}-4 \lambda(\dot{x} N \dot{\omega})^{2}}\right]-\frac{\lambda}{2} m^{2} c^{2} \\
& +\frac{\alpha}{2 \omega^{2}}
\end{aligned}
$$

Repeating the computations made in Section 10.1, we arrive at the Hamiltonian action $\int d \tau p \dot{x}+\pi \dot{\omega}-H$ with the Hamiltonian

$$
\begin{aligned}
H= & \frac{\lambda}{2}\left(p^{2}+m^{2} c^{2}\right)+\frac{1}{2}\left(\pi^{2}-\frac{\alpha}{\omega^{2}}\right)+\lambda_{2}(\omega \pi) \\
& +\lambda_{3}(p \omega)+\lambda_{4}(p \pi)+\lambda_{0} p_{\lambda} .
\end{aligned}
$$

This can be compared with (202). Recall that the constraint $\pi^{2}-\alpha / \omega^{2}=0$ arises as a secondary constraint, from the condition of preservation in time of the primary constraint $\omega \pi=0$. The Hamiltonian action provides both equations of motion and constraints of the vector model in an arbitrary parametrization. Using the reparametrization invariance, we take physical time as the evolution parameter, $\tau=x^{0} / c=t$; then the Hamiltonian action reads

$$
\begin{aligned}
S_{H} & =\int d t c p_{0}+p_{i} \dot{x}^{i}+\pi_{\mu} \dot{\omega}^{\mu} \\
& -\frac{\lambda}{2}\left(-p_{0}^{2}+p_{i}^{2}+m^{2} c^{2}\right)-\frac{1}{2}\left(\pi^{2}-\frac{\alpha}{\omega^{2}}\right)-\lambda_{i} T_{i} .
\end{aligned}
$$

We can treat the term associated with $\lambda$ as a kinematic constraint of the variational problem. According to known prescription of classical-mechanics, we solve the constraint

$$
-p_{0}=p^{0}=\sqrt{\mathbf{p}^{2}+m^{2} c^{2}}
$$


and substitute the result back into (240); this gives an equivalent form of the functional

$$
\begin{aligned}
S_{H} & =\int d t p_{i} \dot{x}^{i}+\pi_{\mu} \dot{\omega}^{\mu}-\left[c \sqrt{\mathbf{p}^{2}+m^{2} c^{2}}\right. \\
& \left.+\frac{1}{2}\left(\pi^{2}-\frac{\alpha}{\omega^{2}}\right)+\lambda_{2} \omega_{\mu} \pi^{\mu}+\lambda_{3} p_{\mu} \omega^{\mu}+\lambda_{4} p_{\mu} \pi^{\mu}\right],
\end{aligned}
$$

where the substitution (330) is implied in the last two terms as well. We have excluded the variables $x^{0}$ and $p_{0}$; the remaining variables are $x^{i}(t), p_{i}(t), \omega^{\mu}(t)$, and $\pi^{\mu}(t)$. The expression in square brackets represents the Hamiltonian. The sign in front of the square root in (330) was chosen according to the right spinless limit. We have excluded nonphysical variables of the position sector and work now with $x^{i}(t), p^{i}(t), \omega^{\mu}(t)$, and $\pi^{\mu}(t)$.

The variational problem implies the first-class constraints $T_{2}=\omega \pi=0, T_{5}=\pi^{2}-\alpha / \omega^{2}=0$ and the second-class constraints

$$
\begin{aligned}
& T_{3}=-p^{0} \omega^{0}+p^{i} \omega^{i}=0, \\
& T_{4}=-p^{0} \pi^{0}+p^{i} \pi^{i}=0 .
\end{aligned}
$$

In all expressions below the symbol $p^{0}$ represents the function (330).

The action (331) implies the Hamiltonian equations

$$
\begin{aligned}
\frac{d x^{i}}{d t} & =c \frac{p^{i}}{p^{0}}, \\
\frac{d p^{i}}{d t} & =0, \\
\dot{\omega}^{\mu} & =\pi^{\mu}+\lambda_{2} \omega^{\mu}+\lambda_{4} p^{\mu}, \\
\dot{\pi}^{\mu} & =-\frac{\omega^{\mu}}{\omega^{4}}-\lambda_{2} \pi^{\mu}-\lambda_{3} p^{\mu} .
\end{aligned}
$$

Equations (333) describe free-moving particle with the speed less then speed of light

$$
x^{i}=x_{0}^{i}+v^{i} t, \quad v^{i}=c \frac{p^{i}}{\sqrt{\mathbf{p}^{2}+(m c)^{2}}}, p^{i}=\text { const. }
$$

The spin-sector variables have ambiguous evolution, because a general solution to (334) depends on an arbitrary function $\lambda_{2}$. So they do not represent the observable quantities. As candidates for the physical variables of spin-sector, we can take either the Frenkel spin-tensor,

$$
\begin{aligned}
\frac{d S^{\mu \nu}}{d t} & =0, \\
S^{\mu \nu} p_{\nu} & =0, \\
S^{2} & =6 \hbar^{2},
\end{aligned}
$$

or, equivalently, the Pauli-Lubanski vector (170)

$$
\begin{aligned}
\frac{d s^{\mu}}{d t} & =0, \\
s^{\mu} p_{v} & =0, \\
s^{2} & =\frac{3 \hbar^{2}}{4} .
\end{aligned}
$$

For the attempt to impose the first-class constraints on statevectors, see [116].

To take into account the second-class constraints $T_{3}$ and $T_{4}$, we construct the Dirac bracket (245). The nonvanishing Dirac brackets are

$$
\begin{aligned}
\left\{x^{i}, x^{j}\right\}_{D} & =\frac{\epsilon^{i j k} s_{k}}{m c p^{0}}=\frac{S^{i j}}{2 m c p^{0}}, \\
\left\{x^{i}, p^{j}\right\}_{D} & =\delta^{i j}, \\
\left\{S^{\mu \nu}, S^{\alpha \beta}\right\}_{D} & =2\left(g^{\alpha[\mu} S^{\nu] \beta}-g^{\beta[\mu} S^{\nu] \alpha}\right), \\
\left\{x^{\mu}, S^{\alpha \beta}\right\}_{D} & =\frac{1}{(m c)^{2}}\left(S^{\mu[\beta} p^{\alpha]}-\frac{p^{\mu}}{p^{0}} S^{0[\beta} p^{\alpha]}\right), \\
\left\{s^{i}, s^{j}\right\}_{D} & =\frac{p^{0}}{m c} \epsilon^{i j k}\left(s_{k}-\frac{(\mathbf{s p}) p_{k}}{p_{0}^{2}}\right), \\
\left\{x^{i}, s^{j}\right\}_{D} & =\left(s^{i}-\frac{(\mathbf{s p}) p^{i}}{p_{0}^{2}}\right) \frac{p^{j}}{(m c)^{2}},
\end{aligned}
$$

where

$$
g^{\mu \nu} \equiv \eta^{\mu \nu}-\frac{p^{\mu} p^{\nu}}{p^{2}}
$$

After transition to the Dirac brackets, the second-class constraints can be used as strong equalities. In particular, we can present $s^{0}$ in terms of independent variables

$$
s^{0}=\frac{(\mathbf{s p})}{\sqrt{\mathbf{p}^{2}+(m c)^{2}}}
$$

and in the expression for Hamiltonian (331) we can omit the last two terms. Besides, we omit the second and third terms, as they do not contribute into equations for observables. In the result, we obtain the physical Hamiltonian

$$
H_{\mathrm{ph}}=c p^{0}=c \sqrt{\mathbf{p}^{2}+(m c)^{2}} .
$$

As it should be, (333), (336), and (337) follow from physical Hamiltonian with the use of Dirac bracket, $\dot{z}=\left\{z, H_{\mathrm{ph}}\right\}_{D}$, where $z=\left(\mathbf{p}, \mathbf{x}, S^{\mu \nu}, s^{\mu}\right)$.

Both operators (except $\widehat{p}_{i}$ ) and abstract state-vectors of the physical time formalism we denote by capital letters, $\widehat{Z}, \Psi(t, \mathbf{x})$. In order to quantize the model, classical Dirac bracket algebra should be realized by operators, $\left[\widehat{Z}_{i}, \widehat{Z}_{j}\right]=$ $\left.i \hbar\left\{z_{i}, z_{j}\right\}_{D}\right|_{z_{i} \rightarrow \widehat{Z}_{i}}$. To find the quantum realization, we first look 
for classical variables which have canonical Dirac brackets, thus simplifying the quantization procedure. We introduce [29] the variables $\widetilde{x}^{j}, \tilde{p}^{j}=p^{j}$, and $\widetilde{s}_{j}$ as follows:

$$
\begin{aligned}
& \tilde{x}^{j}=x^{j}-\frac{1}{m c\left(p^{0}+m c\right)} \epsilon^{j k m} s_{k} p_{m}, \\
& \tilde{s}_{j}=\left(\delta_{j k}-\frac{p_{j} p_{k}}{p^{0}\left(p^{0}+m c\right)}\right) s^{k} ;
\end{aligned}
$$

then the inverse transformation is

$$
\begin{aligned}
& x^{j}=\widetilde{x}^{j}+\frac{1}{m c\left(p^{0}+m c\right)} \epsilon^{j k m} \widetilde{s}_{k} p_{m}, \\
& s_{j}=\left(\delta_{j k}+\frac{p_{j} p_{k}}{m c\left(p^{0}+m c\right)}\right) \widetilde{s}^{k} .
\end{aligned}
$$

Note that in the expression for $x$ and $\widetilde{x}$ we can replace $\widetilde{s} \leftrightarrow$ $s$. We point out that the original and new variables obey the same equations of motion (333) and (337), so they are indistinguishable in the free theory. In an interacting theory their dynamics will be different.

The new variables have canonical algebra with respect to Dirac brackets

$$
\begin{aligned}
& \left\{\tilde{x}^{j}, \tilde{x}^{i}\right\}_{D}=0, \\
& \left\{\widetilde{x}^{i}, p^{j}\right\}_{D}=\delta^{i j}, \\
& \left\{\tilde{x}^{j}, \widetilde{s}^{i}\right\}_{D}=0, \\
& \left\{\tilde{s}^{i}, \tilde{s}^{j}\right\}_{D}=\epsilon^{i j k} \widetilde{s}_{k} .
\end{aligned}
$$

Besides, the constraints (337) on $s^{\mu}$ imply $\widetilde{\mathbf{s}}^{2}=(3 / 4) \hbar^{2}$. So the corresponding operators $\widehat{\widetilde{S}}^{j}$ should realize an irreducible representation of $\mathrm{SO}(3)$ with spin $s=1 / 2$. Quantization in terms of these variables becomes straightforward. The Hilbert space consists of two-component functions $\Psi_{a}(t, \mathbf{x}), a=$ 1,2. A realization of the Dirac brackets by operators has the standard form

$$
\begin{aligned}
& p_{j} \longrightarrow \widehat{p}_{j}=-i \hbar \partial_{j}, \\
& \tilde{x}^{j} \longrightarrow \widehat{\widetilde{X}}^{j}=x^{j}, \\
& \widetilde{s}^{j} \longrightarrow \widehat{\widetilde{S}}^{j}=\frac{\hbar}{2} \sigma^{j} .
\end{aligned}
$$

The conversion formulas (347) between canonical and original variables have no ordering ambiguities, so we immediately obtain the operators $\widehat{X}^{j}$ and $\widehat{S}_{\mathrm{PL}}^{j}$ corresponding to position and Pauli-Lubanski vector of classical theory

$$
x^{i} \longrightarrow \widehat{X}^{i}=x^{i}+\frac{\hbar}{2 m c\left(\widehat{p}^{0}+m c\right)} \epsilon^{i j k} \sigma_{j} \widehat{p}_{k}
$$

$$
\begin{aligned}
s^{j} & \longrightarrow \widehat{S}_{\mathrm{PL}}^{j}=\frac{\hbar}{2}\left(\sigma^{j}+\frac{1}{m c\left(\hat{p}^{0}+m c\right)}(\widehat{\mathbf{p}} \boldsymbol{\sigma}) \hat{p}^{j}\right), \\
\hat{S}_{\mathrm{PL}}^{0} & =\frac{\hbar}{2 m c}(\widehat{\mathbf{p}} \boldsymbol{\sigma}),
\end{aligned}
$$

where the expression for $\widehat{S}_{\mathrm{PL}}^{0}$ follows from (344) and (347). Using (171) and (152) relating the Pauli-Lubanski vector with Frenkel spin-tensor and three-vector of spin, we obtain their quantum realization as follows:

$$
\begin{aligned}
\hat{S}^{0 i} & =-\frac{\hbar}{m c} \epsilon^{i j k} \widehat{p}_{j} \sigma_{k}, \\
\hat{S}^{i j} & =\frac{\hbar}{m c} \epsilon^{i j k}\left(\hat{p}^{0} \sigma_{k}-\frac{1}{\left(\hat{p}^{0}+m c\right)}(\widehat{\mathbf{p}} \boldsymbol{\sigma}) \widehat{p}_{k}\right), \\
\hat{S}^{i} & =\frac{1}{4} \epsilon^{i j k} \widehat{S}_{j k}=\frac{\hbar}{2 m c}\left(\hat{p}^{0} \sigma^{i}-\frac{1}{\left(\hat{p}^{0}+m c\right)}(\hat{\mathbf{p}} \boldsymbol{\sigma}) \hat{p}^{i}\right) .
\end{aligned}
$$

The energy operator (345) determines the evolution of a statevector according to the Schrödinger equation

$$
i \hbar \frac{d \Psi}{d t}=c \sqrt{\widehat{\mathbf{p}}^{2}+(m c)^{2}} \Psi
$$

as well the evolution of operators by Heisenberg equations. The scalar product we define as follows:

$$
\langle\Psi, \Phi\rangle=\int d^{3} x \Psi^{\dagger} \Phi
$$

then

$$
P=\Psi^{\dagger} \Psi
$$

is a probability density for $\widetilde{x}^{i}$. We emphasize that an abstract vector $\Psi(t, \mathbf{x})$ of Hilbert space represents an amplitude of probability density of canonical coordinate $\tilde{x}^{i}$. The wave function for the original coordinate $x^{i}$ should be constructed according to known rules of quantum mechanics.

Let us introduce the operator $\hat{p}^{0}=-i \hbar\left(d / d x^{0}\right)$. Then the Schrödinger equation reads $\left(\widehat{p}^{0}+\sqrt{\widehat{\mathbf{p}}^{2}+(m c)^{2}}\right) \Psi=0$, and applying the operator $\widehat{p}^{0}-\sqrt{\widehat{\mathbf{p}}^{2}+(m c)^{2}}$ we obtain KleinGordon equation

$$
\left(\widehat{p}^{2}+m^{2} c^{2}\right) \Psi=0, \quad \widehat{p}^{2}=\widehat{p}^{\mu} \widehat{p}_{\mu} .
$$

Hence all solutions to the Schrödinger equation form the subspace of positive-energy solutions to the manifestly covariant Klein-Gordon equation for a two-component wave functions.

Let us compare our operators with known in the literature. Pryce [75] studied possible candidates for observables of the Dirac equation; they are marked as $P(d), P(e)$, and $P(c)$ in Tables 3 and 4 . He wrote his operators acting on space of Dirac spinor $\Psi_{D}$; see Table 3. Foldy and Wouthuysen [76] found unitary transformation which maps the Dirac equation $i \hbar \partial_{t} \Psi_{D}=c\left(\alpha^{i} p_{i}+m c \beta\right) \Psi_{D}$ into the pair of square root 
TABLE 3: Position/spin operators for the relativistic electron [75]. $\beta=\left(\begin{array}{cc}1 & 0 \\ 0 & -1\end{array}\right), \alpha^{i}=\left(\begin{array}{cc}0 & \sigma^{i} \\ \sigma^{i} & 0\end{array}\right), \Sigma^{i}=\left(\begin{array}{cc}\sigma^{i} & 0 \\ 0 & \sigma^{i}\end{array}\right)$.

\begin{tabular}{|c|c|}
\hline & Dirac representation, $i \hbar \partial_{t} \Psi_{D}=c\left(\alpha^{i} p_{i}+m c \beta\right) \Psi_{D}$ \\
\hline $\begin{array}{l}\widehat{X}_{P(d)}^{j} \\
\widehat{S}_{P(d)}^{j}\end{array}$ & $\begin{array}{c}x^{j}+\frac{i \hbar}{2 m c} \beta\left(\alpha^{j}-\frac{\alpha^{k} \widehat{p}_{k} \widehat{p}^{j}}{\left(\widehat{p}^{0}\right)^{2}}\right) \\
\frac{1}{2 m^{2} c^{2}}\left(m^{2} c^{2} \Sigma^{j}-i m c \beta \epsilon^{j k l} \alpha_{k} \widehat{p}_{l}\right)\end{array}$ \\
\hline $\begin{array}{l}\widehat{X}_{P(e)}^{j}=\widehat{x}_{\mathrm{FW}}^{j} \\
\hat{S}_{P(e)}^{j}=\widehat{S}_{\mathrm{FW}}^{j}\end{array}$ & $\begin{array}{c}x^{j}+\frac{\hbar}{2 \hat{p}^{0}}\left(i \beta \alpha^{j}+\frac{1}{\hat{p}^{0}+m c} \epsilon^{j k m} \widehat{p}_{k} \Sigma_{m}-\frac{1}{\widehat{p}^{0}\left(\widehat{p}^{0}+m c\right)} i \beta \alpha^{k} \widehat{p}_{k} \widehat{p}^{j}\right) \\
\frac{\hbar}{2 \widehat{p}^{0}}\left(m c \Sigma^{j}-i m \beta \epsilon^{j k l} \alpha_{k} \widehat{p}_{l}+\frac{\Sigma^{k} \widehat{p}_{k} \widehat{p}^{j}}{\widehat{p}^{0}+m c}\right)\end{array}$ \\
\hline $\begin{array}{l}\widehat{X}_{P(c)}^{j} \\
\widehat{S}_{P(c)}^{j}\end{array}$ & $\begin{array}{c}x^{j}+\frac{\hbar}{2\left(\widehat{p}^{0}\right)^{2}}\left(\epsilon^{j k m} \widehat{p}_{k} \Sigma_{m}+i m c \beta \alpha^{j}\right) \\
\frac{\hbar}{2\left(\widehat{p}^{0}\right)^{2}}\left(m^{2} c^{2} \Sigma^{j}-i m c \beta \epsilon^{j k l} \alpha_{k} \widehat{p}_{l}+\Sigma^{k} \widehat{p}_{k} \widehat{p}^{j}\right)\end{array}$ \\
\hline
\end{tabular}

TABLE 4: Position/spin operators for the relativistic electron [75]. $\beta=\left(\begin{array}{cc}1 & 0 \\ 0 & -1\end{array}\right), \Sigma^{i}=\left(\begin{array}{cc}\sigma^{i} & 0 \\ 0 & \sigma^{i}\end{array}\right)$

\begin{tabular}{|c|c|c|}
\hline & F-W representation, $i \hbar \partial_{t} \Psi=c \beta \widehat{p}^{0} \Psi$ & Vector model \\
\hline$\widehat{X}_{P(d)}^{j}$ & $x^{j}-\frac{\hbar}{2 m c\left(\widehat{p}^{0}+m c\right)} \epsilon^{j k m} \widehat{p}_{k} \Sigma_{m}$ & position $x^{j} \longrightarrow \widehat{X}^{j},(350)$ \\
\hline$\widehat{S}_{P(d)}^{j}$ & $\frac{\hbar}{2 m c} \beta\left(\widehat{p}^{0} \Sigma^{j}-\frac{1}{\left(\hat{p}^{0}+m c\right)} \widehat{p}^{k} \Sigma_{k} \hat{p}^{j}\right)$ & Frenkel spin $S^{j} \longrightarrow \widehat{S}^{j},(353)$ \\
\hline $\begin{array}{l}\widehat{X}_{P(e)}^{j}=\widehat{x}_{\mathrm{FW}}^{j} \\
\widehat{S}_{P(e)}^{j}=\widehat{S}_{\mathrm{FW}}^{j}\end{array}$ & $\begin{array}{c}x^{j} \\
\frac{\hbar}{2} \Sigma^{j}\end{array}$ & $\begin{array}{l}\tilde{x}^{j} \longrightarrow \widehat{\widetilde{X}}^{j} \\
\tilde{S}^{j} \longrightarrow \hat{\widetilde{S}}^{j}\end{array}$ \\
\hline$\widehat{X}_{P(c)}^{j}$ & $x^{j}+\frac{\hbar}{2 \widehat{p}^{0}\left(\hat{p}^{0}+m c\right)} \epsilon^{j k m} \widehat{p}_{k} \Sigma_{m}$ & \\
\hline$\widehat{S}_{P(c)}^{j}$ & $\frac{\hbar}{2 \widehat{p}^{0}} \beta\left(m c \Sigma^{j}+\frac{1}{\left(\hat{p}^{0}+m c\right)} \widehat{p}^{k} \Sigma_{k} \widehat{p}^{j}\right)$ & $s^{j} \longrightarrow \widehat{S}_{\mathrm{PL}}^{j},(351)$ \\
\hline
\end{tabular}

equations $i \hbar \partial_{t} \Psi=c \beta \widehat{p}^{0} \Psi$. Applying the FW transformation, the Pryce operators acquire block-diagonal form on space $\Psi$; see Table 4. Our operators act on space of solutions of square root equation (354), so we compared them with positiveenergy parts (upper-left blocks) of Pryce operators in Table 4.

Our operators of canonical variables $\widehat{\widetilde{X}}^{j}=x^{j}$ and $\widehat{\widetilde{S}}^{j}$ correspond to the Pryce $(e)$ ( Foldy-Wouthuysen $\sim$ NewtonWigner [117]) position and spin operators.

However, operators of position $x^{j}$ and spin $S^{j}$ of our model are $\widehat{X}^{j}$ and $\widehat{S}^{j}$. They correspond to the Pryce $(d)$ operators.

Operator of Pauli-Lubanski vector $\widehat{S}_{\mathrm{PL}}^{j}$ is the Pryce (c) spin (we note the normalization of our $s^{j}$; see (170)).

\section{Relativistic Covariance of Canonical Formalism}

While we have started from the relativistic theory (327), working in the physical time parametrization we have lost, from the beginning, the manifest relativistic covariance. Is the quantum mechanics thus obtained a relativistic theory? In particular, will the scalar product (355) and probability (356) be the Lorentz-covariant quantities? Are the mean values $\left\langle\Psi, \widehat{X}^{i} \Phi\right\rangle$ and $\left\langle\Psi, \widehat{S}^{i} \Phi\right\rangle$ the Lorentz-covariant quantities? To answer these questions, we follow the standard ideology of quantum theory.

First, we associate with our theory the manifestly covariant Hilbert space of states $\psi$ which carries a representation of Poincaré group and admits conserved four-vector $\partial_{\mu} I^{\mu}(\psi)=$ 0 with positive null-component. We define the invariant integral over space-like surface $\Omega$ :

$$
\begin{aligned}
P_{\Omega} & =-\frac{1}{6} \int_{\Omega} \epsilon_{\mu \alpha \beta \gamma} J^{\mu} d x^{\alpha} d x^{\beta} d x^{\gamma}, \Longrightarrow \\
\int_{\Omega_{1}} & =\int_{\Omega_{2}},
\end{aligned}
$$

then $P_{t=\text { const }}=\int J^{0} d^{3} x$

can be identified with probability. 
Second, using the covariant formulation (328) of the classical theory, we find quantum realization of basic variables by means of covariant operators acting in this space. The resulting construction is called a covariant formalism.

Third, we establish a correspondence between the canonical and covariant pictures which respect the scalar products (355) and (358) and show that the scalar products, mean values, and transition amplitudes of canonical formalism can be computed using the covariant formalism. This proves the relativistic covariance of quantum mechanics constructed in Section 14.

As we saw above, state-vectors of spinning particle belong to space of solutions to the covariant two-component KleinGordon equation. So it is natural to construct the covariant formalism on this base. We do this in the next subsection. The covariant formalism based on the space of solutions to the Dirac equation will be discussed in Section 15.4.

We emphasize that quantum mechanics of Section 14 already has a clear physical interpretation: the state-vector $\Psi$ describes a spinning particle with positive energy in $\widetilde{x}$-representation, the operator $\widehat{X}$ represents a position, $\widehat{S}$ represents a spin, and so on. Therefore there is no need to search physical interpretation of the covariant formalisms (negative energy states of the Dirac equation and so on), and we will not do it. We consider the covariant formalisms as an auxiliary construction that has the only aim of proving the relativistic covariance of the quantum mechanics formulated in Section 14.

15.1. Relativistic Quantum Mechanics of Two-Component Klein-Gordon Equation. We denote states and operators of covariant formalism by small letters, to distinguish them from the quantities of canonical formalism.

According to Wigner et al. (see [83-85]), with an elementary particle in quantum-field theory we associate the Hilbert space of representation of Poincaré group. The space can be described in a manifestly covariant form as a space of solutions to Klein-Gordon equation for properly chosen multicomponent field $\psi_{a}\left(x^{\mu}\right)$. The space of one-component fields corresponds to spin-zero particle. It is well known that this space has no quantum-mechanical interpretation. In contrast, two-component Klein-Gordon equation does admit the probabilistic interpretation. As we show below, the four-vector (367) represents positively defined conserved current of this equation. Using the current, we can define an invariant scalar product and hence the covariant rules for computing mean values of covariant operators defined on this space.

The two-component KG equation has been considered by Feynman and Gell-Mann [118] to describe weak interaction of spin one-half particle.

Using the Pauli matrices (85) we form the two sets

$$
\begin{aligned}
& \sigma^{\mu}=\left(1, \sigma^{i}\right), \\
& \bar{\sigma}^{\mu}=\left(-1, \sigma^{i}\right) .
\end{aligned}
$$

All the matrices are Hermitian and obey the following rules of permutation of indexes:

$$
\begin{aligned}
& \sigma^{\mu} \bar{\sigma}^{\nu}=-\sigma^{\nu} \bar{\sigma}^{\mu}+2 \eta^{\mu \nu}, \\
& \bar{\sigma}^{\mu} \sigma^{\nu}=-\bar{\sigma}^{\nu} \sigma^{\mu}+2 \eta^{\mu \nu} .
\end{aligned}
$$

Further we define two more sets of $2 \times 2$-matrices:

$$
\begin{aligned}
& \sigma^{\mu \nu}=-\frac{i}{2}\left(\sigma^{\mu} \bar{\sigma}^{\nu}-\sigma^{\nu} \bar{\sigma}^{\mu}\right)=\left(0,-i \sigma^{i}, i \sigma^{i}, \epsilon^{i j k} \sigma^{k}\right), \\
& \bar{\sigma}^{\mu \nu}=-\frac{i}{2}\left(\bar{\sigma}^{\mu} \sigma^{\nu}-\bar{\sigma}^{\nu} \sigma^{\mu}\right)=\left(0, i \sigma^{i},-i \sigma^{i}, \epsilon^{i j k} \sigma^{k}\right) .
\end{aligned}
$$

We have shown the explicit form of their components $00,0 i, i 0$, and $i j$. They are related by Hermitian conjugation, $\sigma^{\mu \nu \dagger}=\bar{\sigma}^{\mu \nu}$, and obey the identities

$$
\begin{aligned}
& \bar{\sigma}^{\alpha} \sigma^{\mu \nu}=\bar{\sigma}^{\mu \nu} \bar{\sigma}^{\alpha}-2 i \eta^{\alpha[\mu} \bar{\sigma}^{\nu]}, \\
& \sigma^{\alpha} \bar{\sigma}^{\mu \nu}=\sigma^{\mu \nu} \sigma^{\alpha}-2 i \eta^{\alpha[\mu} \sigma^{\nu]} .
\end{aligned}
$$

Using them, we verify that both $\sigma^{\mu \nu}$ and $\bar{\sigma}^{\mu \nu}$ obey $\operatorname{SO}(1,3)$ algebra, for instance,

$$
\begin{aligned}
& {\left[\sigma^{\mu \nu}, \sigma^{\alpha \beta}\right]} \\
& \quad=2 i\left(\eta^{\mu \alpha} \sigma^{\nu \beta}-\eta^{\mu \beta} \sigma^{\nu \alpha}-\eta^{\nu \alpha} \sigma^{\mu \beta}+\eta^{\nu \beta} \sigma^{\mu \alpha}\right),
\end{aligned}
$$

so they can be taken as generators of linear representation of the Lorentz group on space of two-component complex columns (Weyl spinors) $\psi=\left(\psi_{1}, \psi_{2}\right)$. Under an infinitesimal Lorentz transformation with the parameters $\omega^{\mu}{ }_{\nu}, \delta x^{\mu}=$ $\omega^{\mu}{ }_{\nu} x^{\nu}$, the column $\psi$ transforms as follows:

$$
\begin{aligned}
\delta \psi & =\frac{i}{4} \omega_{\mu \nu} \sigma^{\mu \nu} \psi, \\
\text { then } \delta \psi^{\dagger} & =-\frac{i}{4} \psi^{\dagger} \bar{\sigma}^{\mu \nu} \omega_{\mu \nu} .
\end{aligned}
$$

Note that the contraction $\psi_{1}^{\dagger} \psi_{1}+\psi_{2}^{\dagger} \psi_{2}$ is not an invariant of the transformation. Using (362), we verify that the quantity $\phi^{\dagger} \bar{\sigma}^{\mu} \psi$ is a four-vector (under the finite transformations $x^{\prime \mu}=$ $\Lambda^{\mu}{ }_{\nu}(\omega) x^{\nu}, \Psi^{\prime}=D(\omega) \Psi$, and $\Psi^{\prime \dagger}=\Psi^{\dagger} D^{\dagger}(\omega)$, where $D=$ $e^{(i / 4) \omega_{\mu \nu} \sigma^{\mu \nu}}, \bar{\sigma}^{\mu}$ is an invariant tensor; i.e., $\left(D^{\dagger} \bar{\sigma}_{\mu} D\right) \Lambda^{\mu}{ }_{\nu}=\bar{\sigma}_{\nu}$; for the proof, see $[17,119])$. If $v_{\alpha}$ is a vector, the combination $\chi=v_{\alpha} \bar{\sigma}^{\alpha} \psi$ transforms with help of $\bar{\sigma}^{\mu \nu}, \delta \chi=(i /$ 4) $\omega_{\mu \nu} \bar{\sigma}^{\mu v} \chi$; then $\delta \chi^{\dagger}=-(i / 4) \chi^{\dagger} \sigma^{\mu v} \omega_{\mu \nu}$. So the quantity $\left(v_{\beta} \bar{\sigma}^{\beta} \phi\right)^{\dagger} \sigma^{\mu}\left(v_{\alpha} \bar{\sigma}^{\alpha} \psi\right)$ turns out to be a vector.

Introducing the space of two-component complex functions $\psi\left(x^{\mu}\right)=\left(\psi_{1}, \psi_{2}\right)$, the generators of Poincaré transformations in this space read

$$
j^{\mu \nu}=\frac{1}{2}\left(x^{\mu} \partial^{\nu}-x^{\nu} \partial^{\mu}\right)+\frac{i}{4} \sigma^{\mu \nu}, \quad \partial^{\mu}=\frac{\partial}{\partial x_{\mu}} .
$$

On the Poincaré invariant subspace selected by twocomponent $\mathrm{KG}$ equation

$$
\left(\widehat{p}^{2}+m^{2} c^{2}\right) \psi=0
$$


we define an invariant and positive-defined scalar product as follows. The four-vector

$$
I^{\mu}[\psi, \phi]=\frac{1}{m^{2} c^{2}}(\bar{\sigma} \widehat{p} \psi)^{\dagger} \sigma^{\mu} \bar{\sigma} \widehat{p} \phi-\psi^{\dagger} \bar{\sigma}^{\mu} \phi
$$

represents a conserved current of (366), that is $\partial_{\mu} I^{\mu}=0$, when $\psi$ and $\phi$ satisfy (366). We define the scalar product by means of invariant integral

$$
(\psi, \phi)=\int_{\Omega} d \Omega_{\mu} I^{\mu}, \quad d \Omega_{\mu}=-\frac{1}{6} \epsilon_{\mu \nu \alpha \beta} d x^{\nu} d x^{\alpha} d x^{\beta},
$$

computed over a space-like three-surface $\Omega$. Using the Gauss theorem for the four-volume contained between the surfaces $\Omega_{1}$ and $\Omega_{2}$, we conclude that the scalar product does not depend on the choice of the surface, $\int_{\Omega_{1}}=\int_{\Omega_{2}}$. In particular, it does not depend on time. So we can restrict ourselves to the hyperplane defined by the equation $x^{0}=$ const; then

$$
(\psi, \phi)=\int d^{3} x I^{0} .
$$

Besides, the scalar product is positive-defined, since

$$
I^{0}[\psi, \psi]=\frac{1}{m^{2} c^{2}}(\bar{\sigma} \hat{p} \psi)^{\dagger} \bar{\sigma} \widehat{p} \psi+\psi^{\dagger} \psi>0 .
$$

So, this can be considered as a probability density of operator $\widehat{\mathbf{x}}=\mathbf{x}$. We point out that transformation properties of the column $\psi$ are in agreement with this scalar product: only if $\psi$ transforms as a (right) Weyl spinor, the quantity $I^{\mu}$ represents a four-vector.

Now we can confirm relativistic invariance of scalar product (355) of canonical formalism. We write

$$
\begin{aligned}
(\psi, \phi) & =\int d^{3} x \frac{1}{m^{2} c^{2}}(\bar{\sigma} \widehat{p} \psi)^{\dagger} \bar{\sigma} \widehat{p} \phi+\psi^{\dagger} \bar{\phi} \\
& =\int d^{3} x\left[\left(\frac{1}{m c} \bar{\sigma} \widehat{p}+i\right) \psi\right]^{\dagger}\left(\frac{1}{m c} \bar{\sigma} \widehat{p}+i\right) \phi \\
& =\langle W \psi, W \phi\rangle,
\end{aligned}
$$

where the operator $W=(1 / m c) \bar{\sigma} \hat{p}+i$ has an inverse, $W^{-1}=\left(1 / 2 \widehat{p}^{0}\right)(i \sigma \widehat{p}+m c)$. The operator $1 / \widehat{p}^{0}$ is well-defined in momentum representation; see the next section. Note also that $W$ and $W^{-1}$ commute with the Schrödinger operator (354). Equation (371) suggests the map of canonical space $\{\Psi\}$ onto subspace of positive-energy solutions of covariant space $\{\psi\}, W^{-1}:\{\Psi\} \rightarrow\{\psi\}, \psi=W^{-1} \Psi$.

The map respects the scalar products (355) and (369) and thus proves relativistic invariance of the scalar product $\langle\Psi, \Phi\rangle,\langle\Psi, \Phi\rangle=\langle W \psi, W \phi\rangle=(\psi, \phi)$.

We note that map $W$ is determined up to an isometry, we can multiply $W$ from the left by an arbitrary unitary operator $U, W \rightarrow W^{\prime}=U W, U^{\dagger} U=1$. Here $\dagger$ denotes Hermitian conjugation with respect to scalar product $\langle$, $\rangle$. It is convenient to remove the ambiguity [120] by requiring the Hermiticity of the operator. Positively defined operator $W^{\dagger} W>0$ has a unique square root, $V=\left(W^{\dagger} W\right)^{1 / 2}$. We write identically $W=P V$, where $P=W V^{-1}$ is unitary, so we can omit $P$ and use $V$ instead of $W$. We compute $W^{\dagger} W=\left(2 \widehat{p}^{0} /(m c)^{2}\right) \bar{\sigma} \widehat{p}$; then

$$
\begin{aligned}
V & =\frac{1}{m c} \sqrt{\frac{\widehat{p}^{0}}{\hat{p}^{0}+m c}}[(\bar{\sigma} \widehat{p})+m c], \\
V^{-1} & =\frac{1}{2 \sqrt{\frac{\hat{p}^{0}\left(\widehat{p}^{0}+m c\right)}{2}}[m c-\sigma \widehat{p}],}
\end{aligned}
$$

and final form of the map between canonical and covariant spaces is

$$
\begin{gathered}
V^{-1}:\{\Psi\} \longrightarrow\{\psi\}, \quad \psi=V^{-1} \Psi, \\
\text { then }\langle\Psi, \Phi\rangle=\langle V \psi, V \phi\rangle=(\psi, \phi) .
\end{gathered}
$$

The transformation between state-vectors induces the map of operators

$$
V^{-1} \widehat{Q} V=\widehat{q},
$$

acting in spaces $\Psi$ and $\psi$. In the next section we use the covariant formulation of the vector model to construct manifestly covariant operators which represent our basic variables in the space of $\psi$. Then we show that the map (374) relates operators of canonical and covariant formalism, thus establishing covariant rules for computation of mean values

$$
\langle\Psi, \widehat{Q} \Phi\rangle=(\psi, \widehat{q} \phi) .
$$

15.2. Covariant Operators of Vector Model. Let us return to the covariant formulation (327) and (328) of the classical theory. To take into account the second-class constraints $T_{3}=$ $p_{\mu} \omega^{\mu}=0$ and $T_{4}=p_{\mu} \pi^{\mu}=0$, we construct the Dirac brackets (245) of the variables $x^{\mu}, p^{\mu}, S^{\mu \nu}$, and $s^{\mu}$. The nonvanishing Dirac brackets are as follows.

Spatial sector:

$$
\begin{aligned}
& \left\{x^{\mu}, x^{\nu}\right\}=-\frac{1}{2 p^{2}} S^{\mu \nu}, \\
& \left\{x^{\mu}, p^{\nu}\right\}=\eta^{\mu \nu}, \\
& \left\{p^{\mu}, p^{\nu}\right\}=0 .
\end{aligned}
$$

Frenkel sector:

$$
\begin{aligned}
& \left\{S^{\mu \nu}, S^{\alpha \beta}\right\} \\
& \quad=2\left(g^{\mu \alpha} S^{\nu \beta}-g^{\mu \beta} S^{\nu \alpha}-g^{\nu \alpha} J^{\mu \beta}+g^{\nu \beta} S^{\mu \alpha}\right), \\
& \left\{x^{\mu}, S^{\alpha \beta}\right\}=\frac{1}{p^{2}} S^{\mu[\alpha} p^{\beta]} .
\end{aligned}
$$

Pauli-Lubanski-sector:

$$
\begin{aligned}
& \left\{s^{\mu}, s^{\nu}\right\}=-\frac{1}{\sqrt{-p^{2}}} \epsilon^{\mu \nu \alpha \beta} p_{\alpha} s_{\beta}=\frac{1}{2} S^{\mu \nu}, \\
& \left\{x^{\mu}, s^{\nu}\right\}=-\frac{s^{\mu} p^{\nu}}{p^{2}}=-\frac{1}{4 \sqrt{-p^{2}}} \epsilon^{\mu \nu \alpha \beta} S_{\alpha \beta}-\frac{s^{\nu} p^{\mu}}{p^{2}} .
\end{aligned}
$$

In (377) it has been denoted $g^{\mu}{ }_{\nu} \equiv \delta^{\mu}{ }_{\nu}-\frac{p^{\mu} p_{v}}{p^{2}}$. 
In the covariant scheme, we need to construct operators $\widehat{x}^{\mu}, \widehat{p}^{\mu}, \widehat{j}^{\mu \nu}, \widehat{s}^{\mu}$ whose commutators

$$
\left[\widehat{q}_{1}, \widehat{q}_{2}\right]=\left.i \hbar\left\{q_{1}, q_{2}\right\}_{D}\right|_{q_{i} \rightarrow \widehat{q}_{i}}
$$

are defined by the Dirac brackets (376)-(380). Inspection of the classical equations $S^{2}=3 \hbar^{2} / 4$ and $p^{2}+(m c)^{2}=0$ suggests that we can look for a realization of operators in the Hilbert space constructed in Section 15.1. tors

With the spin-sector variables we associate the opera-

They obey the desired commutators (381), (379), and (377). To find the position operator, we separate the inner angular momentum $\widehat{s}^{\mu \nu}$ in the expression (365) of Poincaré generator

$$
\begin{aligned}
\widehat{j}^{\mu \nu}= & \frac{1}{2}\left[x^{\mu}+\frac{\hbar(\sigma \widehat{p})^{\mu}}{2 \widehat{p}^{2}}\right] \widehat{p}^{\nu}-\frac{1}{2}\left[x^{\nu}+\frac{\hbar(\sigma \widehat{p})^{\nu}}{2 \widehat{p}^{2}}\right] \widehat{p}^{\mu} \\
& +\frac{i}{4} \widehat{s}^{\mu \nu} .
\end{aligned}
$$

This suggests the operator of "relativistic position"

$$
x^{\mu} \longrightarrow \hat{x}_{\mathrm{rp}}^{\mu}=\widehat{x}^{\mu}+\frac{\hbar}{2 \widehat{p}^{2}}(\sigma \widehat{p})^{\mu},
$$

where $\widehat{x}^{\mu} \psi=x^{\mu} \psi$. The operators $\widehat{p}_{\mu}=-i \hbar \partial_{\mu}$, (382), (383), and (385) obey the algebra (381) and (376)-(380).

15.3. Proof of Relativistic Covariance. Relativistic invariance of the scalar product (355) has been already shown in Section 15.1. Here we show how the covariant formalism can be used to compute mean values of operators of canonical formulation, thus proving their relativistic covariance. Namely, we confirm [29] the following.

Proposition 1. Let

$$
\begin{aligned}
H_{\mathrm{can}}^{+} & =\left\{\Psi(t, \vec{x}) ; i \hbar \frac{d \Psi}{d t}=\sqrt{\widehat{\mathbf{p}}^{2}+(m c)^{2}} \Psi,\langle\Psi, \Phi\rangle\right. \\
& \left.=\int d^{3} x \Psi^{\dagger} \Phi\right\}
\end{aligned}
$$

be Hilbert space of canonical formulation and

$$
\begin{aligned}
& H_{\mathrm{cov}}=\left\{\psi\left(x^{\mu}\right) ;\left(\widehat{p}^{2}+m^{2} c^{2}\right) \psi=0,(\psi, \phi)\right. \\
& =\int_{\Omega} d \Omega_{\mu} I^{\mu}, I^{\mu}=\frac{1}{(m c)^{2}}(\bar{\sigma} \widehat{p} \psi)^{\dagger} \sigma^{\mu} \bar{\sigma} \widehat{p} \phi \\
& \left.-\psi^{\dagger} \bar{\sigma}^{\mu} \phi\right\}
\end{aligned}
$$

be Hilbert space of two-component KG equation. With a statevector $\Psi$ we associate $\psi$ as follows:

$$
\psi=V^{-1} \Psi, \quad V^{-1}=\frac{1}{2 \sqrt{\widehat{p}^{0}\left(\widehat{p}^{0}+m c\right)}}[m c-\sigma \widehat{p}]
$$

Then $\langle\Psi, \Phi\rangle=(\psi, \phi)$. Besides, mean values of the physical position and spin operators (350)-(353) can be computed as follows:

$$
\begin{aligned}
\left\langle\Psi, \widehat{X}^{i} \Phi\right\rangle & =\operatorname{Re}\left(\psi, \widehat{x}_{\mathrm{rp}}^{i} \phi\right), \\
\left\langle\Psi, \widehat{S}^{i j} \Phi\right\rangle & =\left(\psi, \widehat{s}^{i j} \phi\right), \\
\left\langle\Psi, \widehat{S}^{i} \Phi\right\rangle & =\frac{1}{4} \epsilon^{i j k}\left(\psi, \widehat{s}^{j k} \phi\right),
\end{aligned}
$$

where $\hat{x}_{\mathrm{rp}}^{i}$ and $\widehat{s}^{i j}$ are spatial components of the manifestly covariant operators (385) and (383).

It will be convenient to work in the momentum representation, $\psi\left(x^{\mu}\right)=\int d^{4} p \psi\left(p^{\mu}\right) e^{(i / \hbar) p x}$. Transition to the momentum representation implies the substitution

$$
\begin{aligned}
& \widehat{p}_{\mu} \longrightarrow p_{\mu}, \\
& \widehat{x}_{\mu} \longrightarrow i \hbar \frac{\partial}{\partial p^{\mu}},
\end{aligned}
$$

in the expressions of covariant operators (382), (383), (385), and so on.

An arbitrary solution to the KG equation reads

$$
\begin{aligned}
& \psi(t, \mathbf{x})=\int d^{3} p\left(\psi(\mathbf{p}) e^{(i / \hbar) \omega_{p} x^{0}}+\psi_{-}(\mathbf{p}) e^{-(i / \hbar) \omega_{p} x^{0}}\right) \\
& \cdot e^{-(i / \hbar)(\mathbf{p x})}, \\
& p^{0}=-p_{0} \equiv \omega_{p}=\sqrt{\mathbf{p}^{2}+(m c)^{2}},
\end{aligned}
$$


where $\psi(\mathbf{p})$ and $\psi_{-}(\mathbf{p})$ are arbitrary functions of threemomentum; they correspond to positive and negative energy solutions. The scalar product can be written then as follows:

$$
(\psi, \phi)=2 \int \frac{d^{3} p \omega_{p}}{m^{2} c^{2}}\left[\psi^{\dagger}(\bar{\sigma} p) \phi-\psi_{-}^{\dagger}(\sigma p) \phi_{-}\right] .
$$

We see that this scalar product separates positive and negative energy parts of state-vectors. Since our classical theory contains only positive energies, we restrict our further considerations by the positive-energy solutions. In the momentum representation the scalar product (369) reads through nontrivial metric $\rho$ and then through the operator $V$ as follows:

$$
\begin{aligned}
(\psi, \phi) & =\langle W \psi, W \phi\rangle=\left\langle\psi, W^{\dagger} W \phi\right\rangle=\int d^{3} p \psi^{\dagger} \rho \phi \\
& =\langle V \psi, V \phi\rangle=\langle\Psi, \Phi\rangle, \quad \rho=\frac{2 \omega_{p}}{m^{2} c^{2}}(\bar{\sigma} p) .
\end{aligned}
$$

Now our basic space is composed by arbitrary functions $\psi(\mathbf{p})$. The operators $\widehat{x}^{i}, \widehat{s}^{\mu}$, and $\widehat{s}^{\mu \nu}$ act on this space as before, with the only modification that $\widehat{p}^{0} \psi(\mathbf{p})=\omega_{p} \psi(\mathbf{p})$. The operator $\widehat{x}^{0}$ and, as a consequence, the operator $\hat{x}_{\mathrm{rp}}^{0}$ do not act in this space. Fortunately, they are not necessary to prove the proposition formulated above.

Given operator $\widehat{A}$ we denoted its Hermitian conjugated in space $H_{\text {can }}^{+}$as $\widehat{A}^{\dagger}$. Hermitian operators in space $H_{\text {can }}^{+}$ have both real eigenvalues and expectation values. Consider an operator $\hat{a}$ in space $H_{\text {cov }}$ with real expectation values $(\psi, \hat{a} \psi)=(\psi, \hat{a} \psi)^{*}$. It should obey $\hat{a}^{\dagger} \rho=\rho \widehat{a}$. That is, such an operator in $H_{\text {cov }}$ should be pseudo-Hermitian. We denote pseudo-Hermitian conjugation in $H_{\text {cov }}$ as follows: $\widehat{a}_{c}=$ $\rho^{-1} \widehat{a}^{\dagger} \rho$. Then pseudo-Hermitian part of an arbitrary operator $\widehat{a}$ is given by $(1 / 2)\left(\hat{a}+\widehat{a}_{c}\right)$.

Let us check the pseudo-Hermiticity properties of basic operators. From the following identities:

$$
\begin{aligned}
& \left(\sigma^{\mu \nu}\right)^{\dagger} \rho=\rho\left(\sigma^{\mu \nu}+\frac{2 i}{p^{2}}(\sigma p)\left(p^{\mu} \bar{\sigma}^{\nu}-p^{\nu} \bar{\sigma}^{\mu}\right)\right), \\
& \left(\sigma^{\mu \nu} p_{\nu}\right)^{\dagger} \rho=\rho\left(\sigma^{\mu \nu} p_{\nu}+2 i\left[p^{\mu}-(\sigma p) \bar{\sigma}^{\mu}\right]\right), \\
& \left(\hat{x}_{\mathrm{rp}}^{j}\right)^{\dagger} \rho \\
& \quad=\rho\left(\hat{x}_{\mathrm{rp}}^{j}+\frac{i \hbar}{m^{2} c^{2} \omega_{p}}\left[\frac{m^{2} c^{2}}{\omega_{p}} p^{j}-p^{j}(\vec{\sigma} \vec{p})\right]\right),
\end{aligned}
$$

we see that operators $\sigma^{\mu \nu}$ and $\widehat{x}_{\mathrm{rp}}^{j}$ are nonpseudo-Hermitian, while operators $\widehat{p}^{\mu}, \widehat{s}_{\mathrm{PL}}^{\mu}, \widehat{s}^{\mu \nu}$, and orbital part of $\widehat{j}^{i j}$ are pseudoHermitian.

The transformation between state-vectors induces the map of operators

$$
\begin{gathered}
V^{-1} \widehat{Q} V=\widehat{q}, \\
\text { then }\langle\Psi, \widehat{Q} \Phi\rangle=(\psi, \widehat{q} \phi) .
\end{gathered}
$$

Due to Hermiticity of $V, V^{\dagger}=V$, pseudo-Hermitian operators, $\hat{q}^{\dagger} V^{2}=V^{2} \widehat{q}$, transform into Hermitian operators $\widehat{Q}^{\dagger}=$ $\widehat{Q}$. For an operator $\widehat{q}$ which commutes with momentum operator, transformation (374) acquires the following form:

$$
\widehat{Q}=\frac{1}{2}\left(\widehat{q}+\hat{q}^{\dagger}\right)-\frac{1}{2\left(\omega_{p}+m c\right)}\left(\hat{q}-\hat{q}^{\dagger}\right)(\overrightarrow{\sigma p}) .
$$

Using this formula, we have checked by direct computations that covariant operators $\widehat{\mathbf{p}}, \widehat{\boldsymbol{s}}^{\mu \nu}$, and $\widehat{s}_{\mathrm{PL}}^{\mu}$ transform into canonical operators $\widehat{\mathbf{p}}, \widehat{S}^{\mu \nu}$, and $\widehat{S}_{\mathrm{PL}}^{\mu}$ (recall that the spatial part of $\widehat{S}^{\mu \nu}, \widehat{S}^{i}=(1 / 4) \epsilon^{i j k} \widehat{S}_{j k}$ represents the classical spin $\left.S^{i}\right)$. This result together with (375) implies that mean values of these operators of canonical formulation are relativistic-covariant quantities.

Concerning the position operator, we first apply the inverse to (374) to our canonical coordinate $\widehat{\widetilde{X}}^{i}=i \hbar\left(\partial / \partial p^{i}\right)$ in the momentum representation

$$
\begin{aligned}
\widehat{\vec{x}}_{V}^{i}= & V^{-1} \widehat{\widetilde{X}}^{i} V=\widehat{\widetilde{X}}^{i}+\left[V^{-1}, \widehat{\widetilde{X}}^{i}\right] V \\
= & i \hbar \frac{\partial}{\partial p^{i}}-\frac{i \hbar p^{i}(\vec{\sigma} \vec{p})}{2 m c \omega_{p}\left(\omega_{p}+m c\right)}+\frac{i \hbar p^{i}}{2 \omega_{p}}+\frac{i \hbar}{2 m c} \sigma^{i} \\
& +\frac{\hbar}{2 m c\left(\omega_{p}+m c\right)} \epsilon^{i j k} \sigma_{j} p_{k} .
\end{aligned}
$$

Our position operator (347) then can be mapped as follows:

$$
\begin{aligned}
\widehat{x}_{V}^{i}= & V^{-1}\left(i \hbar \frac{\partial}{\partial p^{i}}+\frac{1}{m c\left(\omega_{p}+m c\right)} \epsilon^{i j k} \widehat{S}_{\mathrm{PL} j} p_{k}\right) V \\
= & i \hbar \frac{\partial}{\partial p^{i}}+\frac{i \hbar p^{i}(\overrightarrow{\sigma p})}{2 p^{2} \omega_{p}}+\frac{i \hbar p^{i}}{2 \omega_{p}}-\frac{i \hbar}{2 p^{2}} \omega_{p} \sigma^{i} \\
& +\frac{\hbar}{2 p^{2}} \epsilon^{i j k} p_{j} \sigma_{k} .
\end{aligned}
$$

We note that pseudo-Hermitian part of operator $\hat{x}_{\mathrm{rp}}^{i}$ coincides with the image $\widehat{x}_{V}^{i}$ :

$$
\widehat{x}_{V}^{i}=\frac{1}{2}\left(\hat{x}_{\mathrm{rp}}^{i}+\left[\hat{x}_{\mathrm{rp}}^{i}\right]_{c}\right) .
$$

Since $\widehat{x}_{\mathrm{rp}}^{\mu}$ has explicitly covariant form, this also proves covariant character of position operator $\widehat{X}^{i}$. Indeed, (374) means that matrix elements of $\widehat{X}^{i}$ are expressed through the real part of manifestly covariant matrix elements

$$
\left\langle\Psi, \widehat{X}^{i} \Phi\right\rangle=\left(\psi, \hat{x}_{V}^{i} \phi\right)=\operatorname{Re}\left(\psi, \hat{x}_{\mathrm{rp}}^{i} \phi\right) .
$$

In summary, we have proved the proposition formulated above. It could be formulated also as follows. The operators $\widehat{s}^{\mu \nu}$ and $\hat{x}_{\mathrm{rp}}^{\mu}$, which act on the space of two-component KG equation, represent manifestly covariant form of the Pryce (d)-operators.

Table 5 summarizes manifest form of operators of canonical formalism and their images in covariant formalism. 
TABLE 5: Operators of canonical and manifestly covariant formulations in momentum representation.

\begin{tabular}{lcc}
\hline & Canonical formalism $\Psi(\mathbf{p})$ & Covariant formalism $\psi(\mathbf{p})$ \\
\hline$\widehat{p}_{j} \longrightarrow \hat{p}_{j}$ & $\frac{\hbar}{2 m c}\left(\omega_{p} \sigma^{i}-\frac{1}{\left(\omega_{p}+m c\right)}(\vec{p} \vec{\sigma}) p^{i}\right)$ & $p_{j}$ \\
$\hat{S}^{i} \longrightarrow \hat{s}^{i}$ & $i \hbar \frac{\partial}{\partial p^{i}}-\frac{\hbar}{2 m c\left(\omega_{p}+m c\right)} \epsilon^{i j k} p_{j} \sigma_{k}$ & $\frac{\hbar \omega_{p}}{2(m c)^{2}}\left(\omega_{p} \sigma^{i}-(\vec{p} \vec{\sigma}) p^{i}-i \epsilon_{i m n} p^{m} \sigma^{n}\right)$ \\
$\widehat{X}^{i} \longrightarrow \hat{x}_{V}^{i}$ & $\frac{\hbar}{m c} \epsilon^{i j k}\left(\omega_{p} \sigma_{k}-\frac{1}{\left(\omega_{p}+m c\right)}(\vec{p} \vec{\sigma}) p_{k}\right)$ & $i \hbar \frac{\partial}{\partial p^{i}}+\frac{i \hbar p^{i}(\vec{\sigma})}{2 p^{2} \omega_{p}}+\frac{i \hbar p^{i}}{2 \omega_{p}}-\frac{i \hbar}{2 p^{2}} \omega_{p} \sigma^{i}+\frac{\hbar}{2 p^{2}} \epsilon^{i j k} p_{j} \sigma_{k}$ \\
$\hat{S}^{i j} \longrightarrow \hat{s}^{i j}$ & $\frac{\hbar \omega_{p}}{m^{2} c^{2}} \epsilon^{i j k}\left(\omega_{p} \sigma_{k}-(\vec{p} \vec{\sigma}) p_{k}-i \epsilon_{k m n} p^{m} \sigma^{n}\right)$ \\
$\hat{S}^{0 i} \longrightarrow \hat{s}^{0 i}$ & $-\frac{\hbar}{m c} \epsilon^{i j k} p_{j} \sigma_{k}$ & $-\frac{\hbar}{m^{2} c^{2}} \epsilon^{i j k}\left(\omega_{p} \sigma_{k}-i \epsilon_{k m l} p^{m} \sigma^{l}\right) p_{j}$ \\
$\hat{S}_{\mathrm{PL}}^{0} \longrightarrow \widehat{s}_{\mathrm{PL}}^{0}$ & $\frac{\hbar}{2 m c}(\vec{p} \vec{\sigma})$ & $\frac{\hbar}{2 m c}(\vec{p} \vec{\sigma})$ \\
$\hat{S}_{\mathrm{PL}}^{i} \rightarrow \hat{s}_{\mathrm{PL}}^{i}$ & $\frac{\hbar}{2}\left(\sigma^{i}+\frac{1}{m c\left(\omega_{p}+m c\right)}(\vec{p} \vec{\sigma}) p^{i}\right)$ & $\frac{\hbar}{2 m c}\left(\omega_{p} \sigma^{i}+i \epsilon^{i j k} p_{j} \sigma_{k}\right)$ \\
\hline
\end{tabular}

15.4. Vector Model and Dirac Equation. Here we demonstrate the equivalence of quantum mechanics of two-component Klein-Gordon and Dirac equations. As a consequence, probabilities and mean values of canonical operators (350)-(353) can be computed, using an appropriately constructed covariant operators on the space of Dirac spinors.

Let us replace two equations of second order, (366), by an equivalent system of four equations of the first order. To achieve this, with the aid of the identity $\widehat{p}^{\mu} \widehat{p}_{\mu}=\sigma^{\mu} \widehat{p}_{\mu} \bar{\sigma}^{\nu} \widehat{p}_{\nu}$, we represent (366) in the form

$$
\sigma^{\mu} \widehat{p}_{\mu} \bar{\sigma}^{\nu} \widehat{p}_{\nu} \psi+m^{2} c^{2} \psi=0
$$

Consider an auxiliary two-component function $\bar{\xi}$ (Weyl spinor of opposite chirality) and define evolution of $\psi$ and $\bar{\xi}$ according the equations

$$
\begin{aligned}
\sigma^{\mu} \widehat{p}_{\mu}\left(\bar{\sigma}^{\nu} \widehat{p}_{\nu}\right) \psi+m^{2} c^{2} \psi & =0, \\
\left(\bar{\sigma}^{\nu} \widehat{p}_{\nu}\right) \psi-m c \bar{\xi} & =0 .
\end{aligned}
$$

That is, dynamics of $\psi$ is determined by (401), while $\bar{\xi}$ accompanies $\psi: \bar{\xi}$ is determined from the known $\psi$ taking its derivative, $\bar{\xi}=(1 / m c)(\bar{\sigma} \widehat{p}) \psi$. Evidently, the systems (366) and (402) are equivalent. Rewriting the system (402) in a more symmetric form, we recognize the Dirac equation

$$
\begin{array}{r}
\left(\begin{array}{cc}
0 & \sigma^{\mu} \widehat{p}_{\mu} \\
-\bar{\sigma}^{\nu} \widehat{p}_{\nu} & 0
\end{array}\right)\left(\begin{array}{l}
\psi \\
\bar{\xi}
\end{array}\right)+m c\left(\begin{array}{l}
\psi \\
\bar{\xi}
\end{array}\right)=0, \\
\operatorname{or}\left(\gamma_{W}^{\mu} \widehat{p}_{\mu}+m c\right) \Psi_{\mathrm{DW}}=0
\end{array}
$$

for the Dirac spinor $\Psi_{\mathrm{DW}}=(\psi, \bar{\xi})$ in the Weyl representation of $\gamma$-matrices

$$
\begin{aligned}
& \gamma_{W}^{0}=\left(\begin{array}{ll}
0 & \mathbf{1} \\
\mathbf{1} & 0
\end{array}\right), \\
& \gamma_{W}^{i}=\left(\begin{array}{cc}
0 & \sigma^{i} \\
-\sigma^{i} & 0
\end{array}\right) .
\end{aligned}
$$

This gives one-to-one correspondence among two spaces. With each solution $\psi$ to KG equation we associate the solution

$$
\Psi_{\mathrm{DW}}[\psi]=\left(\begin{array}{c}
\psi \\
\frac{1}{m c}(\bar{\sigma} \widehat{p}) \psi
\end{array}\right)
$$

to the Dirac equation. Below we also use the Dirac representation of $\gamma$-matrices

$$
\begin{aligned}
\gamma^{0} & =\left(\begin{array}{cc}
\mathbf{1} & 0 \\
0 & -\mathbf{1}
\end{array}\right), \\
\gamma^{i} & =\left(\begin{array}{cc}
0 & \sigma^{i} \\
-\sigma^{i} & 0
\end{array}\right) .
\end{aligned}
$$

In this representation, the Dirac spinor corresponding to $\psi$ reads

$$
\begin{aligned}
\Psi_{D}[\psi] & =\frac{1}{\sqrt{2}}\left(\begin{array}{cc}
\mathbf{1} & \mathbf{1} \\
-\mathbf{1} & \mathbf{1}
\end{array}\right)\left(\begin{array}{c}
\psi \\
\frac{1}{m c}(\bar{\sigma} \widehat{p}) \psi
\end{array}\right) \\
& =\frac{1}{\sqrt{2} m c}\left(\begin{array}{c}
{[(\bar{\sigma} \hat{p})+m c] \psi} \\
{[(\bar{\sigma} \hat{p})-m c] \psi}
\end{array}\right) .
\end{aligned}
$$

The conserved current (367) of Klein-Gordon equation (366), being rewritten in terms of Dirac spinor, coincides with the Dirac current (145). Therefore, the scalar product (368) coincides with that of Dirac, $\left(\Psi_{D}, \Phi_{D}\right)_{D}=\int d^{3} x \bar{\Psi}_{D} \gamma^{0} \Psi_{D}$ :

$$
\begin{aligned}
I^{\mu}\left[\psi_{1}, \psi_{2}\right] & =\bar{\Psi}_{D}\left[\psi_{1}\right] \gamma^{\mu} \Psi_{D}\left[\psi_{2}\right], \\
\text { then }(\psi, \phi) & =\left(\Psi_{D}[\psi], \Psi_{D}[\phi]\right)_{D} .
\end{aligned}
$$

This allows us to find manifestly covariant operators in the Dirac theory which have the same expectation values as 
$\widehat{s}^{\mu v}$ and $\widehat{x}_{\mathrm{rp}}^{\mu}$. Consider the following analog of $\widehat{s}^{\mu \nu}$ on the space of 4-component Dirac spinors:

$$
\begin{aligned}
\widehat{s}_{D}^{\mu \nu} & =\hbar \gamma^{\mu \nu}+\hbar \frac{\widehat{p}^{\mu} \gamma^{\nu \alpha} \widehat{p}_{\alpha}-\widehat{p}^{\nu} \gamma^{\mu \alpha} \widehat{p}_{\alpha}}{\widehat{p}^{2}} \\
& =\hbar \gamma^{\mu \nu}+\frac{i \hbar}{\widehat{p}^{2}}\left(\widehat{p}^{\mu} \gamma^{\nu}-\widehat{p}^{\nu} \gamma^{\mu}\right)(\gamma \widehat{p}),
\end{aligned}
$$

where $\gamma^{\mu \nu}=(i / 2)\left(\gamma^{\mu} \gamma^{\nu}-\gamma^{\nu} \gamma^{\mu}\right)$. This definition is independent of a particular representation of $\gamma$-matrices. In the representation (406) this reads

$$
\gamma^{\mu \nu}=\left(\begin{array}{cc}
\sigma^{\mu \nu} & 0 \\
0 & \left(\sigma^{\mu \nu}\right)^{\dagger}
\end{array}\right),
$$

and can be used to prove the equality of matrix elements

$$
\left(\Psi_{D}[\psi] \widehat{s}_{D}^{\mu \nu} \Phi_{D}[\phi]\right)_{D}=\left(\psi, \widehat{s}^{\mu \nu} \phi\right),
$$

for arbitrary solutions $\psi$, $\phi$ of two-component Klein-Gordon equation. The covariant position operator can be defined as follows:

$$
\begin{aligned}
\hat{x}_{D}^{\mu} & =x^{\mu}+\frac{\hbar \gamma^{\mu \alpha} \hat{p}_{\alpha}}{2 \widehat{p}^{2}}+\frac{i \hbar\left(\gamma^{5}-1\right) \hat{p}^{\mu}}{2 \widehat{p}^{2}} \\
& =x^{\mu}+\frac{i \hbar \gamma^{\mu}}{2 \widehat{p}^{2}}(\gamma \widehat{p})+\frac{i \hbar \gamma^{5} \widehat{p}^{\mu}}{2 \widehat{p}^{2}},
\end{aligned}
$$

where $\gamma_{5}=-i \gamma^{0} \gamma^{1} \gamma^{2} \gamma^{3}$. Again, one can check that matrix elements in two theories coincide:

$$
\left(\Psi_{D}[\psi] \hat{x}_{D}^{\mu} \Phi_{D}[\phi]\right)_{D}=\left(\psi, \hat{x}_{\mathrm{rp}}^{\mu} \phi\right) .
$$

As a result, the manifestly covariant operators $\widehat{s}_{D}^{\mu \nu}$ and $\widehat{x}_{D}^{\mu}$ of the Dirac equation represent position $\mathbf{x}$ and spin $\mathbf{S}$ of the spinning particle. Their mean values can be computed as follows:

$$
\begin{aligned}
\left\langle\Psi, \widehat{X}^{i} \Phi\right\rangle & =\frac{1}{2} \operatorname{Re}\left(\Psi_{D}[\psi],\left[\widehat{x}_{D}^{i}+\hat{x}_{D}^{i \dagger}\right] \Phi_{D}[\phi]\right)_{D}, \\
\left\langle\Psi, \widehat{S}^{i} \Phi\right\rangle & =\frac{1}{4} \epsilon^{i j k}\left(\Psi_{D}[\psi], \hat{s}_{D}^{j k} \Phi_{D}[\phi]\right)_{D} .
\end{aligned}
$$

We emphasize that the observables of vector model have an expected behavior both on classical and quantum level. In particular, the position operator $\widehat{X}^{i}$ does not experiences Zitterbewegung, contrary to some other classical models [121]. Note also that the covariant operator (412) that represents the position of spinning particle in the Dirac theory is different from the naive expression used in (149).

15.4.1. The Map $V$ and Foldy-Wouthuysen Transformation. Our map $V$ that relates canonical and two-component KleinGordon spaces turns out to be in close relation with the FoldyWouthuysen transformation. The latter is given by unitary operator

$$
U_{\mathrm{FW}}=\frac{\omega_{p}+m c+(\vec{\gamma} \vec{p})}{\sqrt{2\left(\omega_{p}+m c\right) \omega_{p}}}
$$

and relates the Dirac and four-component Klein-Gordon equations. Applying it to the Dirac spinor $\Psi_{D}[\psi]$, we obtain

$$
U_{\mathrm{FW}} \Psi_{D}[\psi]=\left(\begin{array}{c}
V \psi \\
0
\end{array}\right)=\left(\begin{array}{l}
\Psi \\
0
\end{array}\right) .
$$

That is the operator $V$ is a restriction of $U_{\mathrm{FW}}$ to the space of positive-energy right Weyl spinors $\psi$.

\section{Conclusion}

In nonrelativistic theory, spin can be described on the base of semiclassical Lagrangian (96) which is invariant under spin-plane local symmetry. The symmetry yields two firstclass constraints (93) on spin-sector variables in Hamiltonian formalism. The resulting theory has an expected number of physical degrees of freedom; in particular, the only observable quantities of spin-sector turn out to be the components of spin-vector (92).

To treat spin in a manifestly Lorentz-covariant way, we extended phase space with two auxiliary degrees of freedom, adding null-components to the basic variables $\boldsymbol{\omega}$ and $\pi$. To supply their auxiliary character, we used two secondclass constraints; see (151). This implies drastic modification of the formalism: the constraints induce noncommutative geometry in the phase space even in a free relativistic theory. In particular, spin induces the noncommutative classical brackets (338) of position variables. This must be taken into account in construction of quantum mechanics of a spinning particle. For a spinning electron in Coulomb electric and constant magnetic field, our model yields the nonrelativistic Hamiltonian (259) with correct factors in front of spinorbit and Pauli terms. Hence the spin-induced noncommutativity explains the famous one-half factor in the Pauli equation on the classical level, without appeal to the Thomas precession, Dirac equation, or Foldy-Wouthuysen transformation. Besides, for a spinning body in gravitational field, the spin-induced noncommutativity clarifies the discrepancy in expressions for three-acceleration obtained by different methods; see [33].

Lagrangian of the vector model admits interaction with arbitrary gravitational and electromagnetic fields and has reasonable properties both on classical and on quantum level. Dealing with the variational problem, we were able to determine both Hamiltonian and classical brackets of the theory in unambiguous way.

Regarding the interaction with electromagnetic field, (225)-(227) of our particle with a magnetic moment generalize the approximate equations of Frenkel and BMT to the case of an arbitrary field. Straightforward canonical quantization of the model yields quantum mechanics in Hilbert space of two-component Weyl spinors. All solutions to the Schrödinger equation (354) have positive energy. Since our basic variables obey noncanonical brackets, the operators which represent them have a nonstandard form; see (350) and (353). To establish the relativistic covariance of obtained quantum mechanics, we first developed manifestly relativistic quantum mechanics of two-component Klein-Gordon equation. Then we related states and operators of two formalisms 
and demonstrated on this base the relativistic invariance of the scalar product (355), and relativistic covariance of mean values of operators of canonical formalism; see (373) and (389). Using the relationship (407) between Klein-Gordon and Dirac formalisms, we also formulated the rules (414) for computation the mean values in the framework of Dirac formalism. Here we emphasize once again that we have not tried to find an interpretation of negative energy states presented in the covariant KG and Dirac formalisms. The formalisms were considered as an auxiliary constructions that allow us to prove relativistic covariance of the quantum mechanics formulated in Section 14.

Regarding the interaction with gravity, the minimal coupling gives (314) equivalent to MPTD equations of a rotating body (15)-(17). Spin-gravity interaction induces an effective metric (310) in the Lagrangian equation (312) for a trajectory. To study it in ultrarelativistic limit, we used the Landau-Lifshitz approach to define the three-dimensional geometry and defined on this base the three-dimensional acceleration (57) which guarantees the impossibility for a particle in geodesic motion to overcome the speed of light. The effective metric causes unsatisfactory behavior of MPTD particle in the ultrarelativistic regime. In particular, its acceleration grows with velocity and becomes infinite in the limit. To improve this, we constructed a nonminimal spingravity interaction (282) through a gravimagnetic moment and showed that a fast moving body with unit gravimagnetic moment has a satisfactory behavior.

\section{Conflicts of Interest}

The authors declare that they have no conflicts of interest.

\section{Acknowledgments}

The work of Alexei A. Deriglazov has been supported by the Brazilian foundations $\mathrm{CNPq}$ (Conselho Nacional de Desenvolvimento Científico e Tecnológico, Brazil) and FAPEMIG (Fundação de Amparo à Pesquisa do Estado de Minas Gerais, Brazil). Walberto Guzmán Ramírez thanks CAPES for the financial support (Programme PNPD/2011). The research of Alexei A. Deriglazov was supported by the Tomsk Polytechnic University competitiveness enhancement program.

\section{References}

[1] M. Mathisson, "Neue mechanik materieller systeme," Acta Physica Polonica, vol. 6, pp. 163-200, 1937.

[2] M. Mathisson, "Republication of: new mechanics of material systems," General Relativity and Gravitation, vol. 42, no. 4, pp. 1011-1048, 2010.

[3] V. A. Fock, "On motion of finite masses in general relativity," Soviet Physics-JETP, vol. 9, no. 4, pp. 375-410, 1939.

[4] A. Papapetrou, "Spinning test-particles in general relativity. I," Proceedings of the Royal Society A, vol. 209, no. 1097, pp. 248258, 1951.

[5] W. Tulczyjew, "Motion of multipole particles in general relativity theory binaries," Acta Physica Polonica B, vol. 18, pp. 393409, 1959.
[6] W. G. Dixon, "A covariant multipole formalism for extended test bodies in general relativity," Il Nuovo Cimento, vol. 34, no. 2, pp. 317-339, 1964.

[7] W. G. Dixon, "Classical theory of charged particles with spin and the classical limit of the Dirac equation," Il Nuovo Cimento, vol. 38, no. 4, pp. 1616-1643, 1965.

[8] F. A. E. Pirani, "On the physical significance of the Riemann tensor," Acta Physica Polonica. B, vol. 15, pp. 389-405, 1956.

[9] R. J. Adler, "The three-fold theoretical basis of the Gravity Probe B gyro precession calculation," Classical and Quantum Gravity, vol. 32, no. 22, Article ID 224002, 2015.

[10] A. A. Pomeranskii and I. B. Khriplovich, "Equations of motion of a spinning relativistic particle in external fields," Journal of Experimental and Theoretical Physics, vol. 86, no. 5, pp. 839-849, 1998, Zhurnal Eksperimental'noi i Teoreticheskoi Fiziki, vol. 113, pp. 1537-1557, 1998.

[11] C. M. Will, "The confrontation between general relativity and experiment," Living Reviews in Relativity, vol. 17, no. 4, 2014.

[12] A. B. Balakin and V. A. Popov, "Spin-axion coupling," Physical Review D, vol. 92, no. 10, Article ID 105025, 16 pages, 2015.

[13] J. Frenkel, "Die elektrodynamik des rotierenden elektrons," Zeitschrift für Physik, vol. 37, no. 4-5, pp. 243-262, 1926.

[14] J. Frenkel, "Spinning electrons," Nature, vol. 117, no. 2949, pp. 653-654, 1926.

[15] L. H. Thomas, "The kinematics of an electron with an axis," Philosophical Magazine and Journal of Science, vol. 3, no. 13, supplement 7, p. 1, 1927.

[16] H. C. Corben, Classical and Quantum Theories of Spinning Particles, Holden-Day, San Francisco, Calif, USA, 1968.

[17] A. O. Barut, Electrodynamics and Classical Theory of Fields and Particles, MacMillan, New York, NY, USA, 1964.

[18] J. H. Field, E. Picasso, and F. Combley, "Tests of fundamental physical theories from measurements on free charged leptons," Soviet Physics-Uspekhi, vol. 22, no. 4, pp. 199-219, 1979 (Russian).

[19] J. P. Miller, E. De Rafael, and B. L. Roberts, "Muon (g - 2): Experiment and theory," Reports on Progress in Physics, vol. 70, no. 5, article no. R03, pp. 795-881, 2007.

[20] G. H. Hoffstaetter, H. S. Dumas, and J. A. Ellison, "Adiabatic invariance of spin-orbit motion in accelerators," Physical Review Special Topics - Accelerators and Beams, vol. 9, no. 1, Article ID 014001, 2006.

[21] D. V. Karlovets, "Electron with orbital angular momentum in a strong laser wave," Physical Review A - Atomic, Molecular, and Optical Physics, vol. 86, no. 6, Article ID 062102, 2012.

[22] M. I. Dyakonov and V. I. Perel, "Possibility of orienting electron spins with current," Journal of Experimental and Theoretical Physics Letters, vol. 13, p. 467, 1971.

[23] M. I. Dyakonov, "Magnetoresistance due to edge spin accumulation," Physical Review Letters, vol. 99, no. 12, Article ID 126601, 2007.

[24] A. A. Deriglazov, "Lagrangian for the Frenkel electron," Physics Letters B, vol. 736, pp. 278-282, 2014.

[25] W. G. Ramirez and A. A. Deriglazov, "Lagrangian formulation for Mathisson-Papapetrou-Tulczyjew-Dixon (MPTD) equations," Physical Review D, vol. 92, no. 12, Article ID 124017, 2015.

[26] A. A. Deriglazov and W. G. Ramírez, "Hamiltonian action of spinning particle with gravimagnetic moment," Journal of Physics: Conference Series, vol. 670, Article ID 012020, 2016. 
[27] A. A. Deriglazov and W. G. Ramírez, "Ultrarelativistic spinning particle and a rotating body in external fields," Advances in High Energy Physics, vol. 2016, Article ID 1376016, 27 pages, 2016.

[28] A. A. Deriglazov and W. G. Ramírez, "Mathisson-PapapetrouTulczyjew-Dixon equations in ultra-relativistic regime and gravimagnetic moment," International Journal of Modern Physics D, vol. 26, no. 6, Article ID 1750047, 2017.

[29] A. A. Deriglazov and A. M. Pupasov-Maksimov, "Lagrangian for Frenkel electron and position's non-commutativity due to spin," European Physical Journal C, vol. 74, no. 10, article no. 3101, pp. 1-18, 2014.

[30] A. A. Deriglazov and A. M. Pupasov-Maksimov, "Frenkel electron on an arbitrary electromagnetic background and magnetic Zitterbewegung," Nuclear Physics B, vol. 885, pp. 1-24, 2014.

[31] A. A. Deriglazov and A. M. Pupasov-Maksimov, "Relativistic corrections to the algebra of position variables and spin-orbital interaction," Physics Letters, Section B: Nuclear, Elementary Particle and High-Energy Physics, vol. 761, pp. 207-212, 2016.

[32] A. A. Deriglazov and W. G. Ramírez, "World-line geometry probed by fast spinning particle," Modern Physics Letters A. Particles and Fields, Gravitation, Cosmology, Nuclear Physics, vol. 30, no. 21, Article ID 1550101, 2015.

[33] W. G. Ramírez, A. A. Deriglazov, and A. M. Pupasov-Maksimov, "Frenkel electron and a spinning body in a curved background," Journal of High Energy Physics, vol. 2014, p. 109, 2014.

[34] A. J. Hanson and T. Regge, "The relativistic spherical top," Annals of Physics, vol. 87, pp. 498-566, 1974.

[35] H. Fuchs, "First integrals for the equations of motion for test particles with internal structure," Journal of Mathematical Physics, vol. 32, no. 9, pp. 2473-2477, 1991.

[36] I. B. Khriplovich, "Particle with internal angular momentum in a gravitational field," Soviet Physics-JETP, vol. 69, no. 2, pp. 217-219, 1989.

[37] A. Trautman, "Lectures on general relativity," General Relativity and Gravitation, vol. 34, no. 5, pp. 715-762, 2002.

[38] L. D. Landau and E. M. Lifshitz, The Classical Theory of Fields, Pergamon Press, Oxford, UK, 1980.

[39] A. Galajinsky, "Near horizon geometry of extremal black holes and Banados-Silk-West effect," Physical Review D, vol. 88, Article ID 027505, 2013.

[40] S.-W. Wei, B.-M. Gu, Y.-Q. Wang, and Y.-X. Liu, "Photon emission of extremal Kerr-Newman black holes," European Physical Journal C, vol. 77, no. 2, article no. 128, 2017.

[41] Y.-P. Zhang, B.-M. Gu, S.-W. Wei, J. Yang, and Y.-X. Liu, "Charged spinning black holes as accelerators of spinning particles," Physical Review D, vol. 94, Article ID 124017, 2016.

[42] W.-B. Han and S.-C. Yang, "Exotic orbits due to spin-spin coupling around Kerr black holes," International Journal of Modern Physics D, vol. 26, Article ID 1750179, 2017.

[43] W.-B. Han, "Fast evolution and waveform generator for extreme-mass-ratio inspirals in equatorial-circular orbits," Classical and Quantum Gravity, vol. 33, no. 6, Article ID 065009, 2016.

[44] R. Plyatsko and M. Fenyk, "Antigravity: Spin-gravity coupling in action," Physical Review D - Particles, Fields, Gravitation and Cosmology, vol. 94, no. 4, Article ID 044047, 2016.

[45] B. Mikóczi, "Spin supplementary conditions for spinning compact binaries," Physical Review D, vol. 95, no. 6, Article ID 064023, 2017.

[46] B. Mikóczi, B. Kocsis, P. Forgács, and M. Vasúth, "Parameter estimation for inspiraling eccentric compact binaries including pericenter precession," Physical Review D, vol. 86, no. 10, Article ID 104027, 2012.

[47] M. B. Fröb, "Quantum gravitational corrections for spinning particles," Journal of High Energy Physics, vol. 2016, article 51, 2016, Erratum to: Journal of High Energy Physics, vol. 2016, article 176, 2016.

[48] M. B. Fröb and E. Verdaguer, "Quantum corrections for spinning particles in de Sitter," Journal of Cosmology and Astroparticle Physics, vol. 2017, no. 4, article 022, 2017.

[49] C. Armaza, M. Bañados, and B. Koch, "Can Schwarzschild black holes be accelerators of spinning massive particles?" Classical and Quantum Gravity, vol. 33, no. 10, Article ID 105014, 2016.

[50] C. Armaza, S. A. Hojman, B. Koch, and N. Zalaquett, "On the possibility of non-geodesic motion of massless spinning tops," Classical and Quantum Gravity, vol. 33, no. 14, Article ID 145011, 2016.

[51] A. B. Balakin and V. A. Popov, "Einstein-aether theory: Dynamics of relativistic particles with spin or polarization in a Gödeltype universe," Journal of Cosmology and Astroparticle Physics, vol. 2017, no. 4, article 025, 2017.

[52] K. Wang, Y. F. Zhang, Q. Wang, Z. W. Long, and J. Jing, "Quantum speed limit for relativistic spin- 0 and spin-1 bosons on commutative and noncommutative planes," https://arxiv.org/abs/ 1703.01063.

[53] K. Wang, Y.-F. Zhang, Q. Wang, Z.-W. Long, and J. Jing, "Quantum speed limit for a relativistic electron in the noncommutative phase space," International Journal of Modern Physics A, vol. 32, no. 23-24, Article ID 1750143, 2017.

[54] J. Magueijo and L. Smolin, "Gravity's rainbow," Classical and Quantum Gravity, vol. 21, no. 7, pp. 1725-1736, 2004.

[55] M. A. Gorji, K. Nozari, and B. Vakili, "Gravity's rainbow: A bridge between LQC and DSR," Physics Letters, Section B: Nuclear, Elementary Particle and High-Energy Physics, vol. 765, pp. 113-119, 2017.

[56] M. A. Gorji, K. Nozari, and B. Vakili, "Polymer quantization versus the Snyder noncommutative space," Classical and Quantum Gravity, vol. 32, no. 15, Article ID 155007, 2015.

[57] Z.-W. Feng, S.-Z. Yang, H.-L. Li, and X.-T. Zu, "Thermodynamics and Phase transition of Schwarzschild black hole in Gravity's Rainbow," https://arxiv.org/abs/1608.06824.

[58] S. Weinberg, Lectures on Quantum Mechanics, vol. 1, Cambridge University Press, Cambridge, UK, 2013.

[59] F. A. Berezin and M. S. Marinov, "Classical spin and Grassmann algebra," Journal of Experimental and Theoretical Physics Letters, vol. 21, p. 320, 1975.

[60] F. A. Berezin and M. S. Marinov, "Particle spin dynamics as the grassmann variant of classical mechanics," Annals of Physics, vol. 104, no. 2, pp. 336-362, 1977.

[61] R. D. Pisarski, "Field theory of paths with a curvaturedependent term," Physical Review D-Particles and Fields, vol. 34, no. 2, pp. 670-673, 1986.

[62] A. Deriglazov and A. Nersessian, "Rigid particle revisited: Extrinsic curvature yields the Dirac equation," Physics Letters, Section A: General, Atomic and Solid State Physics, vol. 378, no. 18-19, pp. 1224-1227, 2014.

[63] P. A. M. Dirac, "Generalized Hamiltonian dynamics," Canadian Journal of Mathematics, vol. 2, pp. 129-148, 1950.

[64] P. A. M. Dirac, Lectures on Quantum Mechanics, Yeshiva University, New York, NY, USA, 1964.

[65] D. M. Gitman and I. V. Tyutin, Quantization of Fields with Constraints, Springer, Berlin, Germany, 1990. 
[66] A. Deriglazov, Classical Mechanics: Hamiltonian and Lagrangian Formalism, Springer, 2010.

[67] A. A. Deriglazov, "Analysis of constrained theories without use of primary constraints," Physics Letters. B. Particle Physics, Nuclear Physics and Cosmology, vol. 626, no. 1-4, pp. 243-248, 2005.

[68] M. Bañados and I. Reyes, "A short review on Noether's theorems, gauge symmetries and boundary terms," International Journal of Modern Physics D, vol. 25, no. 10, Article ID 16300214, 74 pages, 2016.

[69] V. A. Borokhov and I. V. Tyutin, "Canonical structure of symmetry transformations in classical mechanics: nondegenerate theories," Physics of Atomic Nuclei, vol. 61, no. 9, pp. 1603-1613, 1998.

[70] V. A. Borokhov and I. V. Tyutin, "Canonical structure of symmetry transformations in classical mechanics: degenerate theories," Physics of Atomic Nuclei, vol. 62, no. 6, pp. 1137-1149, 1999.

[71] A. A. Deriglazov and A. M. Pupasov-Maksimov, "Geometric constructions underlying relativistic description of spin on the base of non-Grassmann vector-like variable," Symmetry, Integrability and Geometry: Methods and Applications, vol. 10, article 012, 13 pages, 2014.

[72] A. A. Deriglazov, "A semiclassical description of relativistic spin without the use of Grassmann variables and the Dirac equation," Annals of Physics, vol. 327, no. 2, pp. 398-406, 2012.

[73] R. P. Feynman, Quantum Electrodynamics, W. A. Benjamin, 1961.

[74] E. Schrödinger, "Uber die kraftfreire Bewegung in der relativistischen Quantunmechanik," Sitzungsber. Preuss. Akad. Wiss, vol. 24, pp. 418-428, 1930.

[75] M. H. Pryce, "The mass-centre in the restricted theory of relativity and its connexion with the quantum theory of elementary particles," Proceedings of the Royal Society. London. Series A. Mathematical, Physical and Engineering Sciences, vol. 195, pp. 62-81, 1948.

[76] L. L. Foldy and S. A. Wouthuysen, "On the dirac theory of spin 1/2 particles and its non-relativistic limit," Physical Review, vol. 78, no. 1, pp. 29-36, 1950.

[77] H. Bauke, S. Ahrens, C. H Keitel, and R. Grobe, "What is the relativistic spin operator?" New Journal of Physics, vol. 16, Article ID 043012, 2014.

[78] M. Wen, H. Bauke, and C. H. Keitel, "Identifying the SternGerlach force of classical electron dynamics," Scientific Reports, vol. 6, Article ID 31624, 2016.

[79] L. C. Céleri, V. Kiosses, and D. R. Terno, "Spin and localization of relativistic fermions and uncertainty relations," Physical Review A - Atomic, Molecular, and Optical Physics, vol. 94, no. 6, Article ID 062115, 2016.

[80] G. N. Fleming, "Nonlocal properties of stable particles," Physical Review, vol. 139, no. 4B, pp. B963-B968, 1965.

[81] E. Harms, G. Lukes-Gerakopoulos, S. Bernuzzi, and A. Nagar, "Spinning test body orbiting around a Schwarzschild black hole: Circular dynamics and gravitational-wave fluxes," Physical Review D - Particles, Fields, Gravitation and Cosmology, vol. 94, no. 10, Article ID 104010, 2016.

[82] D. Kunst, T. Ledvinka, G. Lukes-Gerakopoulos, and J. Seyrich, "Comparing Hamiltonians of a spinning test particle for different tetrad fields," Physical Review D - Particles, Fields, Gravitation and Cosmology, vol. 93, no. 4, Article ID 044004, 2016.
[83] E. Wigner, "On unitary representations of the inhomogeneous Lorentz group," Annals of Mathematics, vol. 40, no. 1, pp. 149204, 1939.

[84] V. Bargmann and E. P. Wigner, "Group theoretical discussion of relativistic wave equations," Proceedings of the National Academy of Sciences of the United States of America, vol. 34, pp. 211-223, 1948.

[85] S. Weinberg, The Quantum Theory of Fields, vol. 1, Cambridge University Press, Cambridge, UK, 1995.

[86] J. D. Jackson, Classical Electrodynamics, John Wiley \& Sons, New York, NY, USA, 1975.

[87] S. S. Stepanov, "Thomas precession for spin and for a rod," Physics of Particles and Nuclei, vol. 43, no. 1, pp. 128-145, 2012.

[88] N. Nicolaevici, "Bouncing Dirac particles: compatibility between MIT boundary conditions and Thomas precession," European Physical Journal Plus, vol. 132, no. 1, article no. 21, 2017.

[89] S. Weinberg, Gravitation and Cosmology, Willey, New York, NY, USA, 1972.

[90] I. B. Khriplovich and A. A. Pomeransky, "Gravitational interaction of spinning bodies, center-of-mass coordinate and radiation of compact binary systems," Physics Letters, Section A: General, Atomic and Solid State Physics, vol. 216, no. 1-5, pp. 7-14, 1996.

[91] M. Dehghani, M. Mardaani, M. Monemzadeh, and S. Abarghouei Nejad, "First class models from linear and nonlinear second class constraints," Modern Physics Letters A, vol. 30, no. 38, Article ID 1550199, 2015.

[92] A. Farmany, M. Hatami, H. Noorizadeh, and S. Mortazavi, "New approach for quantization of second class constraint systems," Journal of Applied Sciences, vol. 14, no. 7, pp. 617-619, 2014.

[93] A. Staruszkiewicz, "Fundamental relativistic rotator," Acta Physica Polonica B, Proceedings Supplement, vol. 1, no. 1, pp. 109-112, 2008.

[94] M. Hajihashemi and A. Shirzad, "A generalized model for the classical relativistic spinning particle," International Journal of Modern Physics A, vol. 31, no. 7, Article ID 1650027, 2016.

[95] Á. Ballesteros, I. Gutiérrez-Sagredo, and P. Naranjo, “On Hamiltonians with position-dependent mass from Kaluza-Klein compactifications," Physics Letters A, vol. 381, no. 7, pp. 701-706, 2017.

[96] A. Stephon and S. Robert, "Axion electron spin quantum mechanics and direct detection," https://arxiv.org/abs/1702.01459.

[97] C. Filgueiras, E. O. Silva, W. Oliveira, and F. Moraes, "The effect of singular potentials on the harmonic oscillator," Annals of Physics, vol. 325, no. 11, pp. 2529-2541, 2010.

[98] E. M. Abreu, B. F. Rizzuti, A. C. Mendes, M. A. Freitas, and V. Nikoofard, "Noncommutative and dynamical analysis in a curved phase-space," Jagellonian University. Institute of Physics. Acta Physica Polonica B, vol. 46, no. 4, pp. 879-903, 2015.

[99] M. Daszkiewicz, "Generalized twist deformations of Poincaré and Galilei quantum groups," Modern Physics Letters A, vol. 30, no. 7, Article ID 1550034, 2015.

[100] M. Daszkiewicz, "Photoelectric effect for twist-deformed spacetime," Jagellonian University. Institute of Physics. Acta Physica Polonica B, vol. 47, no. 5, pp. 1293-1299, 2016.

[101] H. Falomir, P. A. Pisani, F. Vega, D. Cárcamo, F. Méndez, and M. Loewe, "On the algebraic structure of rotationally invariant two-dimensional Hamiltonians on the noncommutative phase space," Journal of Physics A: Mathematical and Theoretical, vol. 49, no. 5, Article ID 055202, 2016. 
[102] S. Pramanik, S. Ghosh, and P. Pal, "Electrodynamics of a generalized charged particle in doubly special relativity framework," Annals of Physics, vol. 346, pp. 113-128, 2014.

[103] V. M. Vasyuta and V. M. Tkachuk, "Classical electrodynamics in a space with spin noncommutativity of coordinates," Physics Letters, Section B: Nuclear, Elementary Particle and High-Energy Physics, vol. 761, pp. 462-468, 2016.

[104] P. Das and S. Ghosh, "Noncommutative geometry and fluid dynamics," European Physical Journal C, vol. 76, no. 11, article no. $627,2016$.

[105] N. Q. Hung and D. Q. Tuan, "Explicit phase space transformations and their application in noncommutative quantum mechanics," VNU Journal of Science: Mathematics_Physics, vol. 31, no. 2, pp. 45-60, 2016.

[106] S. Chanda, G. W. Gibbons, and P. Guha, "Jacobi-Maupertuis metric and Kepler equation," International Journal of Geometric Methods in Modern Physics, vol. 14, no. 7, Article ID 1730002, 2017.

[107] M. Daszkiewicz, "The Henon-Heiles system defined on Liealgebraically deformed Galilei spacetime," Modern Physics Letters A, vol. 32, no. 13, Article ID 1750075, 2017.

[108] M. A. C. Rossi, T. Giani, and M. G. A. Paris, "Probing deformed quantum commutators," Physical Review D - Particles, Fields, Gravitation and Cosmology, vol. 94, no. 2, Article ID 024014, 2016.

[109] K. Ma, "Constrains of charge-to-mass ratios on noncommutative phase space," https://arxiv.org/abs/1705.05789.

[110] K. Ma, Y.-J. Ren, and Y.-H. Wang, "Probing noncommutativities of phase space by using persistent charged current and its asymmetry," https://arxiv.org/abs/1703.10923.

[111] M. Monemzadeh, M. Dehghani, and S. Abarghouei Nejad, "Gauging the relativistic particle model on the noncommutative plane," International Journal of Theoretical Physics, vol. 56, no. 6, pp. 1793-1806, 2017.

[112] Z-S. Zhang, S.-F. Xiao, D.-M. Xun, and Q.-H. Liu, "An enlarged canonical quantization scheme and quantization of a free particle on two-dimensional sphere," Communications in Theoretical Physics, vol. 63, no. 1, article 19, 2015.

[113] D. M. Xun and Q. H. Liu, "Can Dirac quantization of constrained systems be fulfilled within the intrinsic geometry?" Annals of Physics, vol. 341, pp. 132-141, 2014.

[114] E. M. C. Abreu, R. L. Fernandes, A. C. R. Mendes, and J. A. Neto, "Faddeev-Jackiw approach of the noncommutative spacetime Podolsky electromagnetic theory," https://arxiv.org/abs/1705 .03758 .

[115] E. M. C. Abreu and C. F. L. Godinho, "Gauge invariant actions for the noncommutative phase-space relativistic particle," arxiv.org/abs/1701.00800.

[116] T. Rempel and L. Freidel, "A bilocal model for the relativistic spinning particle," Physical Review D, vol. 95, no. 10, Article ID 104014, 2017.

[117] T. D. Newton and E. P. Wigner, "Localized states for elementary systems," Reviews of Modern Physics, vol. 21, no. 3, pp. 400-406, 1949.

[118] R. P. Feynman and M. Gell-Mann, "Theory of the fermi interaction," Physical Review, vol. 109, no. 1, pp. 193-198, 1958.

[119] I. L. Buchbinder and S. M. Kuzenko, Ideas and Methods of Supersymmetry and Supergravity or a Walk Through Superspace, IOP Publishing, 1998.

[120] J. B. Conway, A Course in Functional Analysis, Springer, 1990.
[121] B. F. Rizzuti, E. M. C. Abreu, and P. V. Alves, "Electron structure through a classical description of the Zitterbewegung," Physical Review D - Particles, Fields, Gravitation and Cosmology, vol. 90, no. 2, Article ID 027502, 2014. 


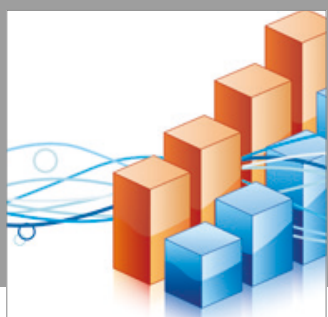

Advances in

Operations Research

vatersals

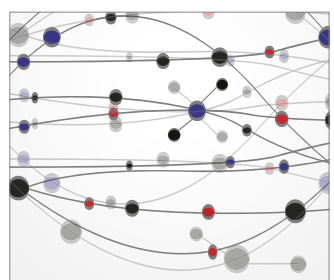

\section{The Scientific} World Journal
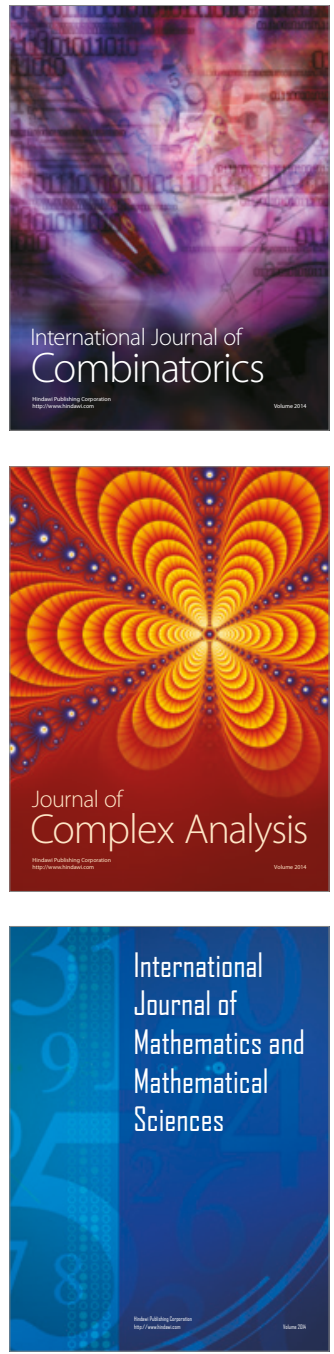
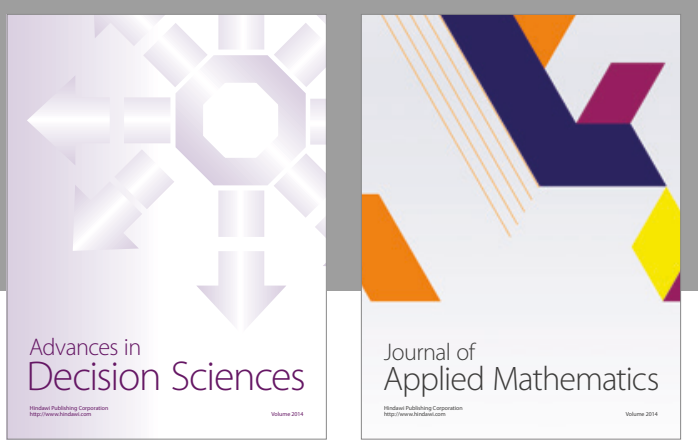

Algebra

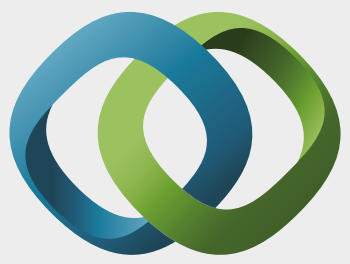

\section{Hindawi}

Submit your manuscripts at

https://www.hindawi.com
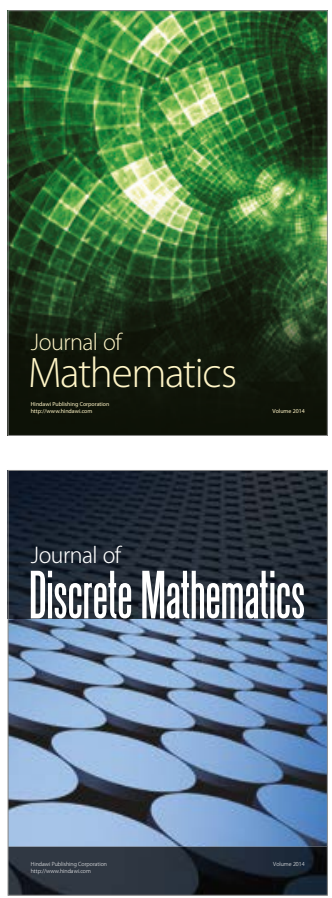

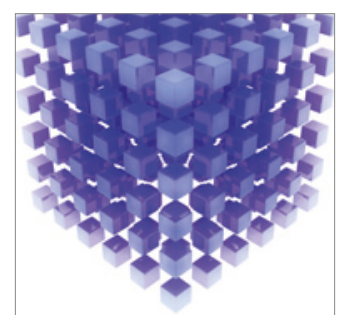

Mathematical Problems in Engineering
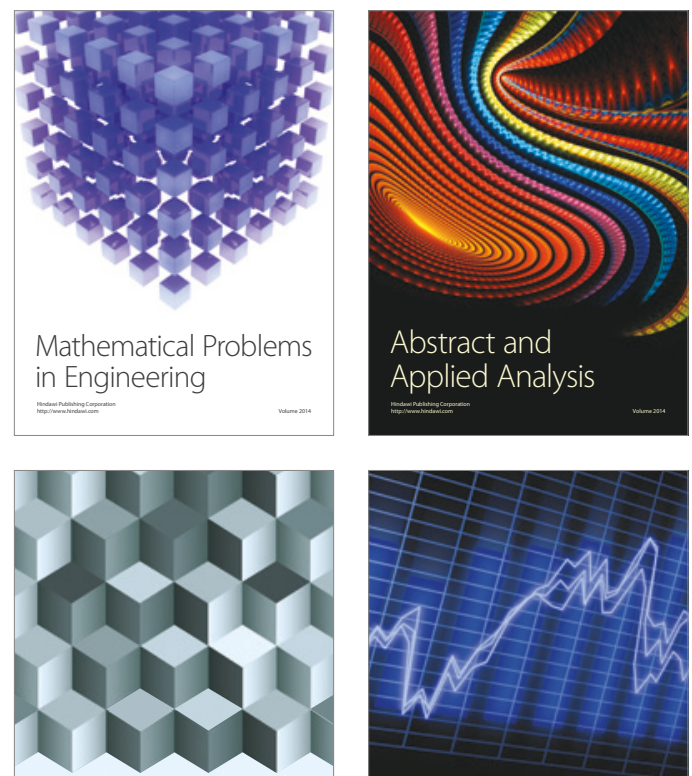

Journal of

Function Spaces

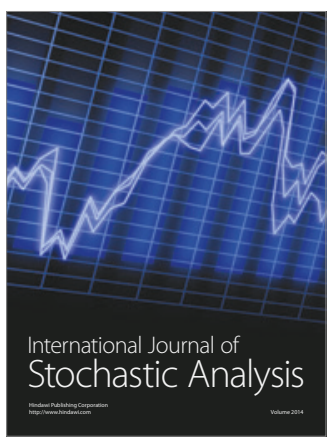

Probability and Statistics
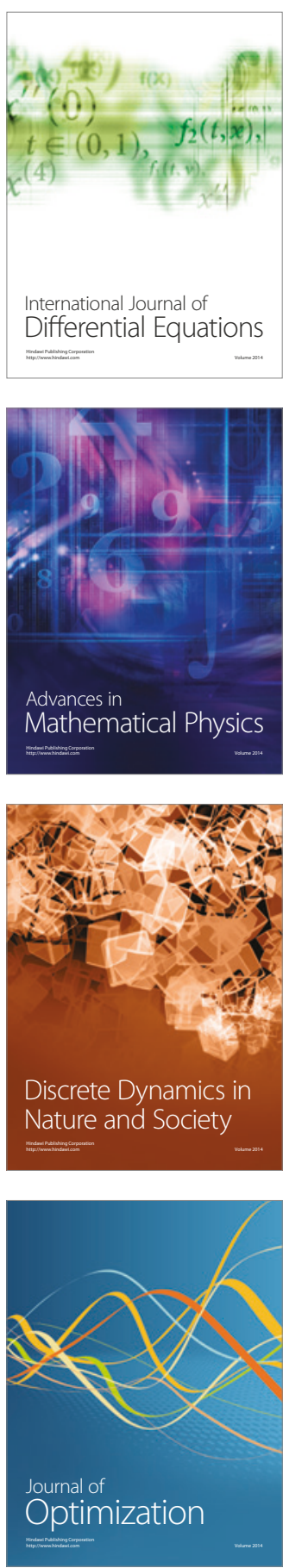